\title{
Polarization modeling and predictions for Daniel K. Inouye Solar Telescope, part 7: preliminary NCSP system calibration and model fitting
}

\author{
David M. Harrington, ${ }^{\mathrm{a}, *}$ Stacey Sueoka, ${ }^{\mathrm{a}}$ Amanda J. White $\odot,{ }^{\mathrm{b}, \mathrm{c}}$ \\ Arthur Eigenbrot $\odot,{ }^{b}$ and Tom Schad $\odot^{a}$ \\ ${ }^{a}$ National Solar Observatory, Makawao, Hawaii, United States \\ ${ }^{b}$ National Solar Observatory, Boulder, Colorado, United States \\ ${ }^{c}$ University of Colorado, Department of Astrophysical and Planetary Sciences, Boulder, \\ Colorado, United States
}

\begin{abstract}
Daniel K. Inouye Solar Telescope (DKIST) is designed to deliver accurate spectropolarimetric solar data across a wide wavelength range, covering a large field of view simultaneously using multiple facility instruments for solar disk, limb, and coronal observations. We show successful design and implementation of National Solar Observatory Coudé Laboratory Spectropolarimeter, a custom metrology tool for efficient continuous broadband polarization calibration of the telescope mirrors through a coudé laboratory focus. We compare multiple fitting techniques for the 10 to $>140$ variable DKIST system polarization models. We compare results with the first DKIST solar calibration observations and find small thermally forced retardance changes of $\pm 0.2 \mathrm{deg}$ and $\pm 0.5 \mathrm{deg}$ for two separate $\mathrm{SiO}_{2}$ retarders. Modulation matrices derived are stable to $< \pm 0.01$ per element during the first on-Sun calibration tests. We achieve good fit agreement to our metrology-based model over a 390- to 1600-nm bandpass. The solutions are robust and efficient using only 10 input Stokes vectors from elliptical calibration retarders. We developed a custom polarizer assembly used with metrology tools to orient the DKIST polarization coordinates to better than 0.1-deg clocking angle. (1) The Authors. Published by SPIE under a Creative Commons Attribution 4.0 Unported License. Distribution or reproduction of this work in whole or in part requires full attribution of the original publication, including its DOI. [DOI: 10 .1117/1.JATIS.7.1.018004]
\end{abstract}

Keywords: instrumentation; polarization; Mueller matrix; Daniel K. Inouye Solar Telescope.

Paper 20161 received Oct. 31, 2020; accepted for publication Mar. 12, 2021; published online Mar. 30, 2021.

\section{Introduction: DKIST and Polarization Models for Calibration}

The National Science Foundation's Daniel K. Inouye Solar Telescope (DKIST) on Haleakalā, Maui, Hawai'i, is presently transitioning from the end of construction to an early operations phase. The telescope has a 4.2 -m-diameter off-axis $F / 2$ primary mirror with a 4.0 -m-diameter circular beam. Four separate spectropolarimeters (SPs) are being installed in the coudé laboratory, each with multiple sensors of various imaging capabilities and wavelength ranges. ${ }^{1-4}$ All instruments have spectral resolving power above 30,000 and many achieve more than 300,000 in certain cases. Each instrument has optomechanical methods to allow for stepping the instrument field of view across a much larger portion of the DKIST delivered beam field of view. All instruments provide imaging spectropolarimetry of some kind either by scanning through wavelengths with Fabry-Perot systems, stepping slit masks across a focal plane, tilting field scanning mirrors near pupil planes, and/or using imaging fiber bundles. These techniques create imaging spectropolarimetric capability over visible- and near-infrared wavelengths. Many science cases require strictly simultaneous observation of several spectral lines with multiple instruments at the same region of the Sun. DKIST is specified to operate up to eight polarimetric cameras simultaneously at frame rates of at least $40 \mathrm{~Hz}$ to achieve the combined spatial, spectral, and temporal

*Address all correspondence to David M. Harrington, dharrington@nso.edu 
polarimetric goals. Accurate polarization calibration (PolCal) of this instrument suite is a critical scientific performance parameter.

We show here that the design and implementation of a custom dual-channel SP we call the National Solar Observatory Coudé Laboratory Spectropolarimeter (NCSP). We deployed this metrology tool on the telescope to calibrate the DKIST system optics polarization for wavelengths between 390 and $1600 \mathrm{~nm}$ with continuous coverage at subnanometer sampling. We also developed an alignment polarizer that was then used on to derive absolute polarization coordinates for the DKIST calibration optics. The NCSP system was used along with the visible broadband imager (VBI) in an on-Sun observing campaign to assess this absolute polarization coordinate reference.

DKIST uses six mirrors to collect and relay light to a rotating coudé lab to provide flexible capabilities. ${ }^{2,5-11}$ The first two mirrors, comprising the off-axis Gregorian telescope, are static with respect to the alt/az telescope mount. DKIST has a Gregorian Optical System (GOS) built around the secondary Gregorian focus. The GOS contains a level for apertures and stops, calibration targets at Gregorian focus. The GOS also contains retarders, polarizers, and artificial light sources (ALSs) at other levels roughly 300 to $500 \mathrm{~mm}$ ahead of the Gregorian focus. ${ }^{8,12-17}$ Four polarimetric instruments presently spanning the 380 - to 5000 -nm wavelength range are in various phases of construction or installation on the summit in the coudé lab. The visible spectropolarimeter (ViSP) is a three-arm slit-based SP. The visible tunable filter (VTF) is a tunable Fabry-Perot type imaging SP. ${ }^{18,19}$ The Diffraction-Limited Near-Infrared Spectropolarimeter (DL-NIRSP) is a fiber-bundle fed imaging SP. The Cryogenic Near-Infrared Spectropolarimeter is also a slit-based infra-red optimized system. We also have two high-speed $(30 \mathrm{~Hz}), 4 \mathrm{k}$ fullframe cameras within the VBI-red and VBI-blue instruments. ${ }^{20-26}$

Three static mirrors level and collimate the beam in the coudé laboratory (M7 to M9). Then a sequence of dichroic beam splitters, windows, and/or mirrors called the Facility Instrument Distribution Optics (FIDO) allows changing of instrument configurations on a timescale of less than half an hour. The FIDO optics allow simultaneous operation of three polarimetric instruments optimized for 380 to $1800 \mathrm{~nm}$ while using the facility adaptive optics (AO) system for correction to provide diffraction limited performance. ${ }^{8,9,19,27,28}$ All AO-assisted instruments see the first beamsplitter in the wavefront correction (WFC) system in transmission. The optics are designed such that the wedge angles are matched in each optic, and every instrument sees either 2 or 4 beamsplitters in transmission to compensate for the wedge and associated wavelength variation in beam deflection. Cryo-NIRSP $(\mathrm{CN})$ can receive all wavelengths to $5000 \mathrm{~nm}$ but without use of the AO system in a seeing limited, all-reflective beam path fed by a FIDO pick-off mirror called M9a.

All instruments are supported by the DKIST data center. The center handle 3000 terabytes of new data per year, with $8 \mathrm{~TB}$ expected on an average day. ${ }^{29-31}$ We show here the successful testing of the PolCal modules written for processing DKIST data. We refer the reader to recent papers outlining the various capabilities of the first-light instruments. ${ }^{1,2,2,4,6,8,9}$ Complex polarization modulation and calibration strategies are required for multi-instrument astronomical systems. ${ }^{8,9,12,13,32,33}$ The planned 4-m on-axis European Solar Telescope will also require similar calibration considerations. ${ }^{34-36}$ Many solar and night-time telescopes have performed PolCal of complex many-mirror pathways. ${ }^{37-60}$

This paper is part of a series investigating polarization performance expectations for the DKIST instruments. We outlined the DKIST optical layout and system Mueller matrix properties when using a simple enhanced silver mirror coating model by Harrington and Sueoka 2017 (HS1 $17^{61}$ ). We showed PolCals of a night time telescope with a ViSP using the daytime sky by Harrington et al. 2017 ( $\left.\mathrm{H} 17^{62}\right)$. We applied the Berreman calculus to polarization fringes formed in multi-layer crystals with predictions and data collected in the lab and at a solar telescope by Harrington et al. $2018\left(\mathrm{H}_{1} 8^{63}\right){ }^{64,65}$ We then extended this calculus by Harrington and Sueoka 2018a (HS18a ${ }^{63}$ ) to include fringe magnitude estimates in converging and diverging beams. We recently have investigated spatial variation of retardance across multi-layer retarders made of polished crystals, stretched polycarbonate, and ferro-electric liquid crystals by

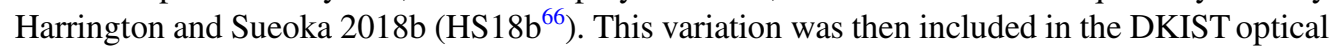
model to show PolCal errors as functions of field angle and wavelength. We extend the coating efforts of $\mathrm{HS} 7^{61}$ to many mirror types from multiple vendors, highly enhanced metal coatings,

J. Astron. Telesc. Instrum. Syst. $\quad 018004-2 \quad$ Jan-Mar 2021 • Vol. 7(1) 
hundred-layer dichroic coatings, and our system of beam splitters by Harrington et al. 2019 $\left(\mathrm{H} 19^{67}\right)$. We show additional mirror measurements and new ellipsometric metrology from an outside vendor (J.A. Woollam) in this paper at a range of incidence angles matching the DKIST mirrors to improve the system polarization model.

Spectral interference fringes adversely impact polarization accuracy for astronomical instruments. Many authors have worked toward optical fringe modeling and/or removal methods either through optical design or data processing. ${ }^{47,48,59,68-79}$ We designed and built several newly upgraded polarization optics based on polycarbonate and optically contact crystal designs in Harrington et al. $2020\left(\mathrm{H} 20^{80}\right)$. These retarders suppress polarization fringes by one to three orders of magnitude compared to the previously designed multi-layer crystal optics. We also identified alignment errors between individual crystals as a major source of error. Misalignment produces spectral oscillations in retardance, which introduce coupling between temperature changes and the spectral drift of these elliptical retardance oscillations. We show here detection of the clocking oscillations outlined in $\mathrm{H} 20^{80}$ with calibrations both on-Sun and with the DKIST calibration lamp. We show here successful on-Sun use of the optically contacted calibration retarder (CalRet) and system calibration with time-efficient calibration sequences (CSs). We demonstrate efficient modulation with NCSP using a copy of the polycarbonate modulator deployed in our lab metrology tool as well as in DL-NIRSP. This modulator combines with a complex optical feed using 10 mirrors, multiple lenses, and fiber fed spectrographs to provide a representative optical system for demonstrating our calibration process.

\subsection{Elements of a System Model: Articulated Mirror Groups}

$$
\mathbf{M}=\left(\begin{array}{cccc}
I I & Q I & U I & V I \\
I Q & Q Q & U Q & V Q \\
I U & Q U & U U & V U \\
I V & Q V & U V & V V
\end{array}\right) .
$$

Most large modern telescopes are articulated in azimuth and elevation and require a pointingdependent system model. PolCal requires removing the influence of the telescope mirrors. The Mueller matrix is the $4 \times 4$ matrix that transfers Stokes vectors. ${ }^{81-83}$ Each element of the Mueller matrix is denoted by a transfer coefficient. ${ }^{83,84}$ For instance, the coefficient $[0,1]$ in the first row transfers $Q$ to $I$ and is denoted $Q I$. The first row terms are denoted $I I, Q I, U I$, and $V I$. The first column of the Mueller matrix elements are $I I, I Q, I U, I V$. In this paper, we will use the notation in Eq. (1). The output Stokes vector is related to the input vector via a simple transfer equation $\mathbf{S}_{\text {out }}=\mathbf{M S}_{\text {in }}$ using standard matrix multiplication. With this formalism, the Stokes vector from some patch of solar atmosphere would be transferred by the Mueller matrix of each optic between the Sun and the sensor.

There are six mirrors that collect the solar flux and relay the beam to the DKIST coude laboratory. When modeling the telescope as a time-dependent system tracking targets in azimuth-elevation coordinates, we create a physical model for the groups of mirrors installed between the various rotation axes. We adopt a notation where a rotation is denoted as $\mathbb{R}$. There are three main coordinate rotations in the DKIST system model. The elevation axis is between M4 and M5 $\left(\mathbb{R}_{\mathrm{EI}}\right)$. The azimuth axis is between M6 and M7 $\left(\mathbb{R}_{\mathrm{Az}}\right)$. The DKIST coudé laboratory sits entirely on a rotating platform, so there is a separate rotational degree of freedom with the coudé table angle $\left(\mathbb{R}_{\mathrm{TA}}\right)$ in addition to the azimuth of the target $\left(\mathbb{R}_{\mathrm{Az}}\right)$. They combine to create the relative angle (Az-TA) in the rotation matrix $\left(\mathbb{R}_{\mathrm{Az}-\mathrm{TA}}\right)$ between M6 and the first coudé mirror (M7). We also include the static 90 deg rotation between the M1:M2 mirror group tilt axes and the M3:M4 mirror group tilt axes as $\left(\mathbb{R}_{23}\right)$ :

$$
\begin{gathered}
\mathbf{S}_{\text {coude }}=\mathbb{R}_{\text {Az-TA }} \mathbf{M}_{6} \mathbf{M}_{5} \mathbb{R}_{\mathrm{El}} \mathbf{M}_{4} \mathbf{M}_{3} \mathbb{R}_{23} \mathbf{M}_{2} \mathbf{M}_{1} \mathbf{S}_{\text {input }}, \\
\mathbf{S}_{\text {coude }}=\mathbb{R}_{\text {Az-TA }} \mathbf{M}_{5: 6} \mathbb{R}_{\mathrm{El}} \mathbf{M}_{3: 4} \mathbb{R}_{23} \mathbf{M}_{1: 2} \mathbf{S}_{\text {input }} .
\end{gathered}
$$

We can transfer the Stokes vectors from upstream of the DKIST primary mirror to the Stokes vectors incident on M7 in the coudé laboratory floor. Equation (2) shows the input Stokes vector 
$\mathbf{S}_{\text {input }}$ being transferred from ahead of the primary mirror to the coudé laboratory $\mathbf{S}_{\text {coude }}$ just before reflection off M7. A fundamental assumption of the "group model" is that the static optics can have their Mueller matrices be multiplied together and fit with a greatly reduced number of variables. For the present modeling efforts, we also ignore all field of view dependence though we can calculate magnitudes and make the models more complex as needed. We show the group model in Eq. (3):

$$
\begin{gathered}
\mathbf{M}_{\mathrm{GOS}}=\mathbb{R}_{-\mathrm{ret}} \mathbf{M}_{\mathrm{ret}} \mathbb{R}_{\text {ret }} \mathbb{R}_{\text {-pol }} \mathbf{M}_{\mathrm{pol}} \mathbb{R}_{\mathrm{pol}}, \\
\mathbf{S}_{\text {coude }}=\mathbb{R}_{\mathrm{Az}-\mathrm{TA}} \mathbf{M}_{5: 6} \mathbb{R}_{\mathrm{El}} \mathbf{M}_{3: 4} \mathbb{R}_{23} \mathbf{M}_{\mathrm{GOS}} \mathbf{M}_{1: 2} \mathbf{S}_{\text {input }} .
\end{gathered}
$$

We note that a rotation of a Mueller matrix must include rotations on both sides of the matrix to preserve the local coordinate systems: $\mathbb{R}(-\theta) \mathbf{M} \mathbb{R}(\theta)$. The single sided rotations of Eqs. (2) and (3) rotate the coordinate frame sequentially. We insert and rotate the calibration polarizer (CalPol) and CalRet ahead of the Gregorian focus as part of the GOS. We use Eq. (4) to define the Mueller matrix for the GOS as a combination of the polarizer rotated into local coordinates by the angle denoted as "pol" and an elliptical retarder rotated into local coordinates by the angle denoted as "ret." We discuss the sequence of polarization states generated by the GOS during calibration in more detail later. This CS can include polarizer-only or retarder-only configurations. The GOS calibration optics combine with the telescope when inserted as per Eq. (5). We note that measurement of modulated flux for each state in a CS is commonly called a PolCal. We show more details of the individual optic Mueller matrices in Appendix D. A single PolCal combined with a database of mirror polarization responses accounting for M1 to M6 can be used to derive an instrument modulation matrix. However, when calibrating the polarization response of the entire system (from M1 through the instrument), a series of many PolCals must be collected to separate the articulated mirror polarization from instrument modulation.

\subsection{Elements of a System Model: Modulation Matrix as a Mirror Group and Instrument}

The modulation matrix is commonly defined as $n$ modulation states by four Stokes vector components $(I Q U V)$ multiplying the incident Stokes vector to create a column vector of detected intensities (i). ${ }^{48,51-53,85-92}$ Equation (6) shows this matrix multiplication. We use subscripts $(I Q U V)$ in the first index of the modulation matrix $(O)$ to denote which Stokes vector component is being modulated. The numerical second index $(1,2, \ldots, m)$ denotes the modulation state 1 through $m$ corresponding to the modulating retarder setting (orientation, voltage, etc):

$$
\left(\begin{array}{c}
i_{1} \\
i_{2} \\
\ldots \\
i_{n}
\end{array}\right)=\left(\begin{array}{cccc}
O_{I 1} & O_{Q 1} & O_{U 1} & O_{V 1} \\
O_{I 2} & O_{Q 2} & O_{U 2} & O_{V 2} \\
\ldots & \ldots & \ldots & \ldots \\
O_{I n} & O_{Q m} & O_{U m} & O_{V m}
\end{array}\right)\left(\begin{array}{c}
I \\
Q \\
U \\
V
\end{array}\right) .
$$

We create a physical model for all the optics contributing to a modulation matrix from M7 through the instrument sensor. We show a Mueller matrix propagation through a DKIST instrument starting with the beam leaving M6 incident on the coudé lab after rotation into the coudé frame by the azimuth and table angles. We also apply the mirror grouping to all the mirrors in the optical relay and within instruments. We refer the reader to more detailed examples of the DKIST optical configuration in Sec. 1.1 of H19. ${ }^{67}$ We outline an example here with the CN instrument optical path. We use this optical path as NCSP also uses these same optics for models presented in this paper. The seventh DKIST mirror (M7) folds the beam parallel to the lab floor. The eighth mirror (M8) is an off axis collimating mirror. The ninth mirror (M9) is a coma correcting fold mirror with a specific figure. The $\mathrm{CN}$ does not use the DKIST AO system presently. The $\mathrm{CN}$ beam path uses a pickoff flat mirror called M9a at 9-deg incidence angle to direct light to the instrument. The next mirror in the system is a steering mirror with a $20-\mathrm{m}$ radius of curvature located near a pupil conjugate plane. We denote this optic as the cryo steering mirror (CSM) working at 4-deg incidence angle. This is followed by the off axis mirror focusing the beam at 
$F / 18$ using a 1.1-deg fold angle denoted CFM. Equation (7) shows the mirror Mueller matrices transferring the coudé lab Stokes vector to the beam ahead of NCSP where the first pickoff mirror is inserted to divert the beam into NCSP:

$$
\mathbf{S}_{\text {cryo }}=\mathbf{M}_{\mathrm{CFM}} \mathbf{M}_{\mathrm{CSM}} \mathbf{M}_{9 \mathrm{a}} \mathbf{M}_{9} \mathbf{M}_{8} \mathbf{M}_{7} \mathbf{S}_{\text {coude }}=\mathbf{M}_{\text {eff }} \mathbf{S}_{\text {coude }} .
$$

The mirrors on the coudé laboratory floor ahead of the modulator have their Mueller matrices grouped together and combined with the appropriate SP. A common technique used at many observatories is to ensure that most folds are either in the horizontal or vertical $(Q)$ plane along combined with a $Q$ analyzer such that the strong and spectrally complex $U V$ retardance terms of coated mirrors and dichroics do not strongly degrade modulation efficiency. By also using this technique, many optics can be grouped and simplified to a few variables in physical model fits. For the CN optical path, we would group all mirrors of Eq. (7) for the optics ahead of the modulator. We then add a rotating elliptical modulator and also combine several more optics behind the modulator inside the spectrograph. All these optic Mueller matrices get combined and propagated through a linear polarizing analyzer. The flux transmitted through that analyzer for each of the Stokes vector components become elements of the modulation matrix.

\section{NSO Coudé Spectropolarimeter for DKIST Metrology}

We designed and built the NCSP to be independent of any complex data processing software required to reduce data from the DKIST instrument suite. Using commercial off-the-shelf fiber fed spectrographs, we can demonstrate DKIST calibrations continuously over an ultra-violet (UV) to near-infrared (NIR) wavelength range with far less complex analysis and minimal development. The NCSP is installed in the DKIST coudé laboratory and is aligned to the optical bore-sight of the telescope similar to the other instruments. A series of fold mirrors pick off the beam converging at $F / 18$ within the $\mathrm{CN}$ instrument mirror path. We drop the beam down below the DKIST coudé beam height and package this $\mathrm{CN} F / 18$ focus on a small optical bench containing a system quite similar to our NLSP described in $\mathrm{HS} 18 \mathrm{~b},{ }^{66} \mathrm{H} 19,{ }^{67}$ and $\mathrm{H} 20{ }^{80}$ Figure 1 shows a CAD layout of the beam starting in the coudé laboratory with DKIST M7, collimated by M8 and folded to a pupil image inside the $\mathrm{CN}$ optics. We refer the reader to Ref. 1 for a high-level overview of the DKIST optical systems, images of the coudé laboratory, and in particular

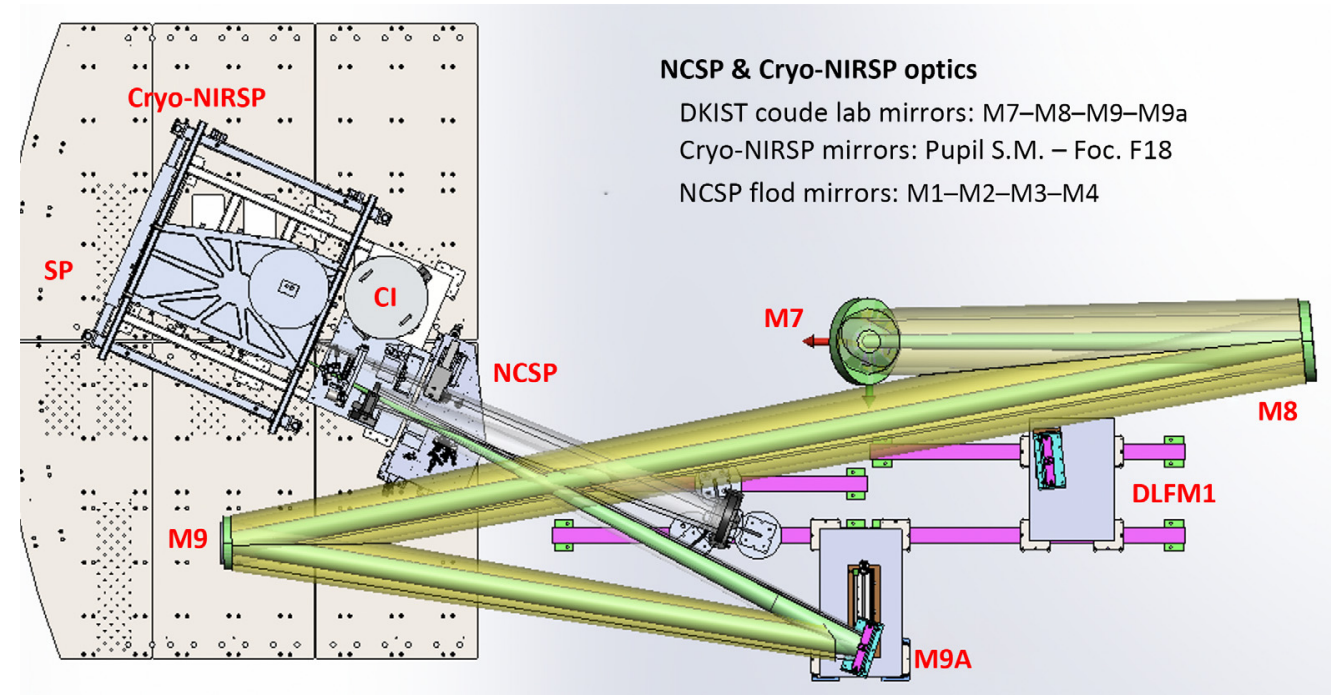

Fig. 1 A CAD model of the DKIST coudé laboratory with the optical beam shown in yellow beginning with reflection off $M 7$ and ending with reflection off M9a sending the beam toward the $C N$ instrument optics. The $\mathrm{CN}$ path is shown with the light gray solid beam. We denote the $\mathrm{CN} \mathrm{Cl}$ and SP. We also annotate the firstfold mirror inside DL-NIRSP as DL-FM1 though unused in this configuration. For reference, the distance between M8 and M9 is 10 with $6 \mathrm{~m}$ between M9 and M9a. 


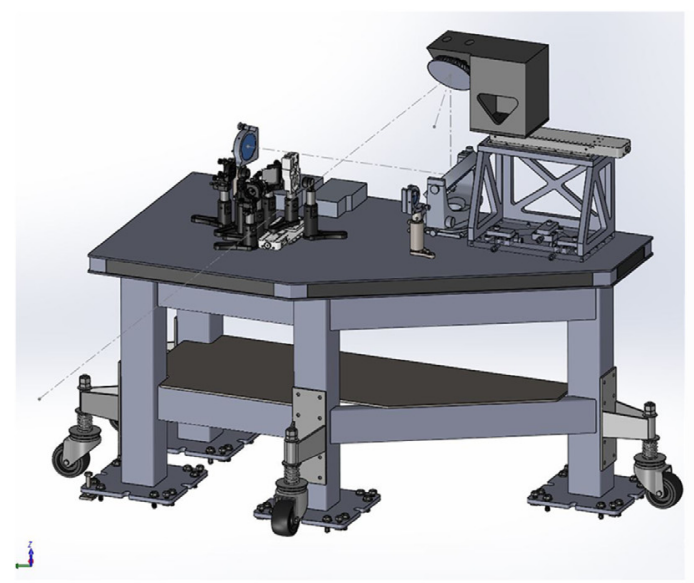

(a)

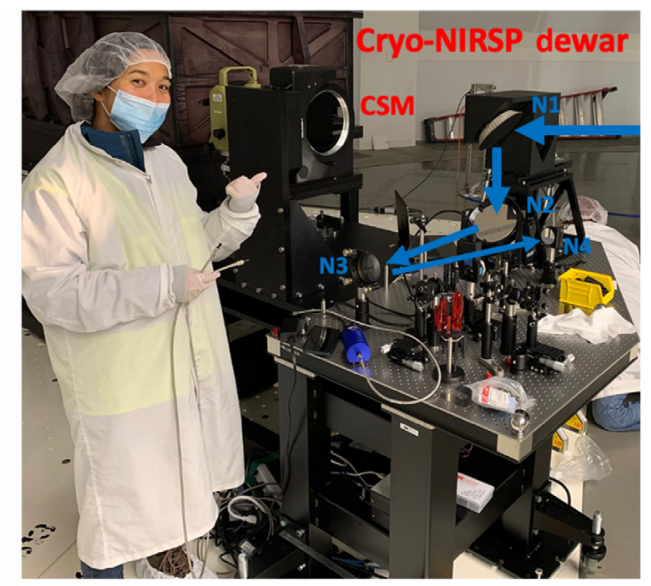

(b)

Fig. 2 (a) The NCSP CAD model starting with the first NCSP pick-off fold mirror sending the beam from incoming horizontal to descending vertical. (b) The table and optics during NCSP installation in the coudé lab along with co-author Sueoka. The CN spectrograph dewar is the large black ribbed metal structure behind Sueoka. The CSM is at the nominal coudé beam height. The beam is picked off by the NCSP fold mirror 1, denoted as N1. The subsequent NCSP fold mirrors are denoted in blue with the optical beam indicated as the blue arrows.

Fig. 9 showing NCSP installed immediately in front of the CN instrument. We show an annotated CAD model in Fig. 1 for the beam beginning with DKIST M7 and ending inside NCSP.

A CAD model of the NCSP optomechanical layout is shown in Fig. 2(a). Figure 2(b) shows the NCSP optical bench during summit installation. A light-weighted elliptical aperture fold mirror is on a large, motorized translation stage for remote control of this first NCSP pickoff mirror.

NCSP uses two spectrographs to simultaneously measure polarized spectra using a wire grid polarizing beam splitter and rotating polycarbonate retarder. The DKIST beam is collimated by an achromatic doublet lens after being stopped to a circular beam of 1.5 - $\mathrm{mm}$ diameter using laser cut masks. This provides a narrow-field, uniform, and collimated light source. A rotating thirdwave linear retarder is mounted as a modulator. The final optic is a fixed orientation analyzing wire grid polarizer also used as a polarizing beam splitter. As detectors, we use a visible wavelength spectrograph (VIS) and a NIR wavelength spectrograph from OceanOptics. The NCSP VIS spectrograph covers 380 to $1100 \mathrm{~nm}$ on 1044 active pixels, whereas the NIR spectrograph covers 900 to $1650 \mathrm{~nm}$ wavelength on 512 pixels.

The beam transmitted through the wire grid polarizer feeds the visible spectrograph via filters, aperture stops, and a lens. At the lens focus, a fiber couples light to the spectrograph. The beam reflected off the wire grid polarizer is passed through a separate set of filters, an additional polarizer, an aperture stop, and lens focusing on a fiber connected to the NIR spectrograph. This NIR arm additional polarizer has wires parallel to the analyzer to remove the fresnel reflection component from the polarizer glass substrate to maintain high contrast (see Appendix $\mathrm{C}$ for the polarization modeling of the NCSP optical path).

The VIS system has spectral sampling of $0.8 \mathrm{~nm}$ per pixel at $370 \mathrm{~nm}$ wavelength falling to $0.7 \mathrm{~nm}$ per pixel at $1100 \mathrm{~nm}$. The NIR system has an average sampling of $1.65 \mathrm{~nm}$ per pixel. We use a 50- $\mu \mathrm{m}$ wide slit on both spectrographs. We use gas discharge (arc) lamps to measure wavelength accuracy and spectral resolving power. We fit roughly $1.8 \mathrm{~nm}$ full-width half-maximum (FWHM) in Gaussian fits to the optical profile on the VIS system and $3.6 \mathrm{~nm}$ on the NIR system. We achieve a sampling of roughly 2 to 3 pixels per optical FWHM on both systems at spectral resolving powers of a few hundred and moderate wavelength dependence. The vendor provides a third-order fit to several arc lines as their wavelength solution. We independently verified 38 spectral lines and measured peak-to-peak variations of $<1 \mathrm{~nm}$. Both systems were assessed for timing and background stability with our custom MATLAB control scripts. We also verified linearity and adjust our peak count level to be well within the sensor linear range. An assessment 


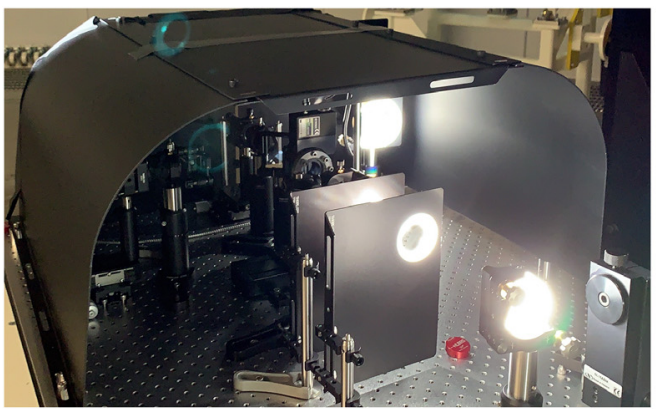

(a)

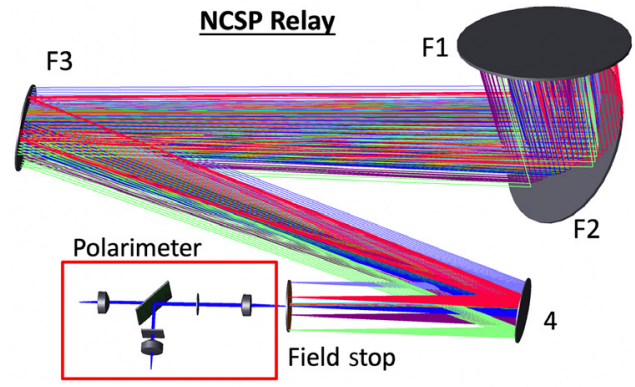

(b)

Fig. 3 (a) NCSP during on-Sun observations in August 2020. The NCSP fold mirrors 3 and 4 are saturated and fully illuminated. (b) An optical Zemax model starting with the first NCSP fold mirror and using the 2.8-arc min field stop at Gregorian focus. The $\sim 2.9$-arc sec field angle beam is collimated by the lens and modulated by the retarder. The 2 -in. square wire grid polarizer is seen at 45-deg incidence splitting the two orthogonal linear states. The reflected beam goes to the NIR channel where an additional wire grid polarizer is oriented parallel to the analyzed state to remove the fresnel reflections and preserve high contrast.

of internal scattered light using a set of lasers showed that the VIS system had a full width at $1 \%$ max count rate of 9 pixels for all but the shortest wavelengths. Provided we use appropriate color balancing filters (see $\mathrm{H} 19^{67}$ ), we will not be substantially impacted by internal scattered light.

Figure 3(a) shows the NCSP system illuminated by roughly $70 \mathrm{~W}$ of optical power from the DKIST solar beam. The bright illuminated mirrors are NCSP fold mirrors 3 and 4 through multiple baffles converging toward the $F / 18$ focus. Figure $3(\mathrm{~b})$ shows the Zemax optical design roughly oriented as in (a). The NCSP fold mirrors 3 and 4 are illuminated by the solar beam, whereas fold mirorrs 1 and 2 are just out of the image. Two drilled metal plates with a rough surface and black anodization were used as additional heat-dissipating stops ahead of the $F / 18$ focus for the on-Sun observing campaign where $>70 \mathrm{~W}$ of optical power are concentrated in the $F / 18$ focus.

\subsection{NCSP Modulation Matrix: Grouping 10 Mirrors}

$$
\mathbf{S}_{\mathrm{NCSP}}=\mathbf{M}_{\mathrm{ER}} \mathbf{M}_{L} \mathbf{M}_{N 4} \mathbf{M}_{N 3} \mathbf{M}_{N 2} \mathbf{M}_{N 1} \mathbf{S}_{\text {cryo }} .
$$

We chose to install NCSP within the $\mathrm{CN}$ optical path using some of the $\mathrm{CN}$ mirrors. We show here the combined polarization model for the four NCSP mirrors that direct the $F / 18 \mathrm{CN}$ solar beam to the NCSP focus including the optical path internal to the NCSP. The six mirrors from Eq. (7) ahead of NCSP are grouped with the four NCSP mirrors. We include the collimating lens $\left(\mathbf{M}_{L}\right)$ ahead of the NCSP modulator, which is modeled as a rotating elliptical retarder $\left(\mathbf{M}_{\mathrm{ER}}\right)$. Equation (8) lists these six NCSP optics. The combined 10 mirrors, lens, and rotating modulator of Eqs. (7) and (8) represent all optics ahead of the analyzing polarizer that combine to impact the NCSP modulation matrix and modulation efficiency calculations (see Appendix C for more detailed polarization modeling of these optics).

\section{DKIST Polarization Absolute Reference Frames}

An external polarization reference frame tied to solar coordinates is required to provide accurate orientations for the derived Stokes vector. We have multiple DKIST CalPols and anticipate infrequent cleaning, polarizer replacement, and possible future upgrades. There are also concerns about rotary stage absolute positioning and error checking that would benefit from having an independent optical verification. We designed an optic that provides a stable long-term polarization reference frame. This includes precise optical referencing of the polarization axis and 
interfaces to laser tracker-based metrology systems, which include the M1:M2 tilt axes and the global telescope coordinate systems.

\subsection{DKIST Master Polarizer: Laser Trackers and Summit Coordinates}

The absolute polarizer orientation reference for DKIST is set by an optic called the master polarizer. This master polarizer is a Moxtek UVT240A fused silica (FS) substrate wire grid polarizer with a 4-nm $\mathrm{Al}_{2} \mathrm{O}_{3}$ conformal protective coating on the wires. This polarizer was bonded wires down into a special cell, modified to include three spherically mounted retroreflectors (SMRs) for reference with the DKIST laser tracker metrology tools and coordinate system. We detail below a laboratory procedure where we can orient the transmission axis of the polarizer ( $Q$ Stokes axis) to these SMRs and the associated SMR nests bonded directly onto the polarizer cell using a laser tracker and a coordinate measuring machine (CMM) arm. We note that we have detailed spatial and spectral variation measurements for several wire grid polarizers in transmission, contrast, and extinction axes to ensure errors in polarizer performance are small compared to alignment errors. The polarizer transmission axis is well oriented in absolute space as installed in the rotary stage as attached to the telescope referenced with the laser tracker. We can then orient the DKIST CalPols to the shared tilt axis of the DKIST primary and secondary mirrors (M1:M2) using the summit laser tracker and the associated DKIST system global coordinate references to tolerances. This master polarizer was placed in a rotary stage on the top most GOS level furthest away from Gegorian focus, in the same level as the ALS. This level is one level above the suite of CalPols and two levels above the retarders. As the master polarizer is mounted ahead of the CalPols, we can put both polarizers in the beam at the same time and search for an intensity null between crossed polarizers. This defines the motor position for each the CalPol, which creates the null. This null position allows us to place the CalPol into the absolute telescope coordinates with the $+Q$ transmission axis aligned with the M1:M2 tilt plane. These absolute coordinates are used to transfer the DKIST coordinate system into solar coordinates establishing a tolerance on the DKIST measured polarization frame relative to solar coordinates.

We show the master polarizer in a laboratory setup in Fig. 4(a). Figure 4(b) shows the two laser tracker positions required to view the master polarizer mounted in the beam ahead of the Gregorian focus. This location can sight the DKIST relay mirrors M3:M4:M5:M6 and the absolute telescope coordinate system reference point as the crossing of the telescope azimuth axis and elevation axis.

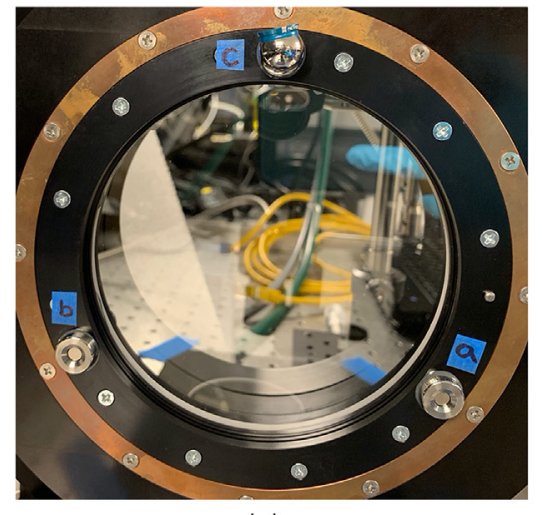

(a)

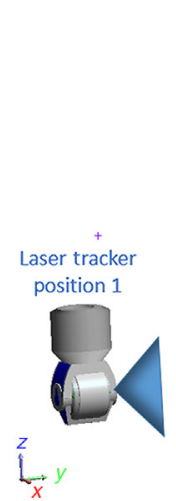

$L_{x} y$

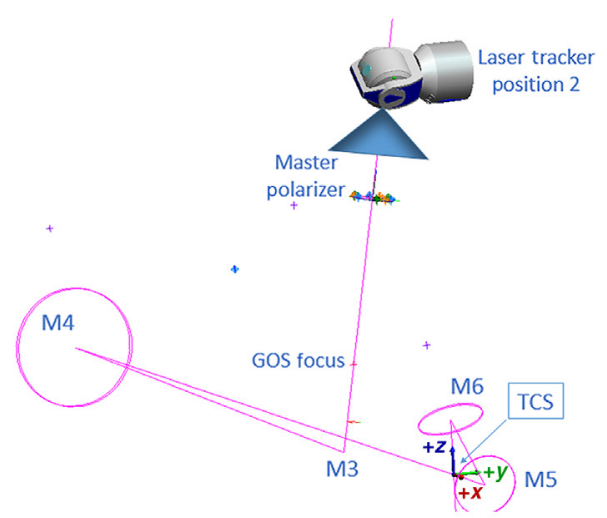

(b)

Fig. 4 (a) The master polarizer along with the SMR nests and a single SMR in a laboratory setup used to establish the polarizer $+Q$ transmission axis against the SMR locations. (b) The summit laser tracker setup used to establish absolute coordinate references of the master polarizer in the DKIST telescope coordinate system. Laser tracker position 1 has visibility to several SMR locations and the absolute telescope coordinate system reference as the crossing of the elevation axis and the azimuth axis. The laser tracker position 2 looks through the GOS box entrance aperture to view the master polarizer as mounted ahead of the CalPols and the GOS focus. 


\subsection{Master Polarizer: Lab Metrology for Extinction Axis and On-Summit Installation}

We created a laboratory setup for the master polarizer to provide for accurate determination of the optic polarization axis relative to the SMR laser tracker balls before deployment to the summit. This lab characterization is the basis for optical knowledge of the master polarizer extinction axis. We created a collimated beam of well-known linear polarization using a Fresnel reflection. A 75-mm-diameter uncoated BK7 window was mounted flush on an optical bench. The back surface was painted black to extinguish the scatter from the bench. The optical beam was mounted near Brewsters angle of $56 \mathrm{deg}$ off the surface of the window to create a very highly polarized beam of well-known linear polarization orientation. We also note that the contrast ratio and extinction axis of these wire grids is well preserved even at high incidence angles. We used our fiber coupled light source and collimating optics from NLSP with a known low elliptical degree of polarization (see $\mathrm{H} 19^{67}$ ). We then reflect this beam off the BK7 uncoated window to create the high-accuracy linear polarization state. The beam is then collected by the receiver end of our NLSP system using the achromatic doublet lens and optical fiber. The fiber feeds the visible wavelength Avantes spectrograph (VIS) from NLSP where we record 380 to $1100 \mathrm{~nm}$ in $>1300$ spectral pixels simultaneously.

The intensity minima occur near $120 \mathrm{deg}$ and $300 \mathrm{deg}$ orientation of the rotary stage encoder. We took measurements at $0.1 \mathrm{deg}$ steps from $109 \mathrm{deg}$ to $135 \mathrm{deg}$ and $289 \mathrm{deg}$ to $315 \mathrm{deg}$. The spectral pixels were binned by $20 \times$ resulting in 66 binned wavelengths covering the bandpass for each orientation measurement. Of the 66 , we chose to use 13 bins with larger count levels covering the bandpass. Of these wavelengths, we recover the polarizer orientation by fitting a parabola to the data set. The measurement setup was repeated a few times with and without Thor Labs blackout foil between the optical table and the glass. We found the same polarizer orientation within a measurement uncertainty as a standard deviation of deg with peak to peak variation below $0.1 \mathrm{deg}$. A tolerance analysis showed that this mild input elliptical polarization is highly extinguished by a Fresnel reflection at Brewsters angle, creating an error well below $0.03 \mathrm{deg}$ in measurement with a $<2 \%$ elliptically polarized input beam. Thus we use the \pm 0.04 deg tolerance for this term. We also have characterized the stabilized lab light source and find that the duration of the testing should not substantially impact the measurement fit. We note that the expected polarizer spatial variation of the extinction axis is smaller than $\pm 0.03 \mathrm{deg}$.

The laboratory measurement uncertainty for the BK7 window surface was $\pm 0.01 \mathrm{deg}$. The spectrograph angle determination uncertainty was $\pm 0.04 \mathrm{deg}$. The angular difference from the laboratory frame to the GOS frame is attributed uncertainty of $\pm 0.05 \mathrm{deg}$. Following an onsummit metrology campaign, we derive the $+Q$ orientation for this master polarizer by setting the rotary stage to $-8.59 \mathrm{deg}$ with an uncertainty of $\pm 0.065 \mathrm{deg}$ for $1 \sigma$ root-sum-square (RSS) combination of these three independent errors.

\subsection{Alignment of the Calibration Polarizers with VBI and MasterPol}

The master polarizer was initially used to align the FS CalPol1 Spare combined with the VBI blue channel using a 430-nm wavelength filter. The VBI blue had an Andor Balor 4k CMOS cameras which was used to collect flux. This VBI channel is mostly unpolarized as there is no analyzer in VBI and low polarization from the internal optics. ${ }^{20-26}$ We defocused the VBI camera significantly to avoid having any significant spatial structure in the images. We also note that the GOS polarizers are mounted well away from any instrument focal planes and thus are well away from conjugate to the VBI image plane. We fit the detected flux versus CalPol angle to detect the minimum in intensity through the crossed polarizers at GOS. The master polarizer was oriented in its stage at $-8.59 \mathrm{deg}$ (counter clockwise looking down) representing transmission along the $+Y$ telescope coordinate axis (master polarizer wires perpendicular to M1 tilt axis). This should create a beam oriented orthogonal to the M1:M2 polarization plane $\pm 0.065 \mathrm{deg}$ with which a GOS CalPol can cross (null intensity). The motor rotation position for null intensity will align the GOS CalPol transmission axis $(+Q)$ along the M1:M2 mirror group tilt axis $(+Q$ absolute coordinates) with the CalPol wires orthogonal to transmission. There were three data sets collected by DKIST tracking the Sun in the 3:47- to 4:37-pm timeframe on August 15, 2020. The Balor

J. Astron. Telesc. Instrum. Syst. $\quad 018004-9 \quad$ Jan-Mar 2021 • Vol. 7(1) 


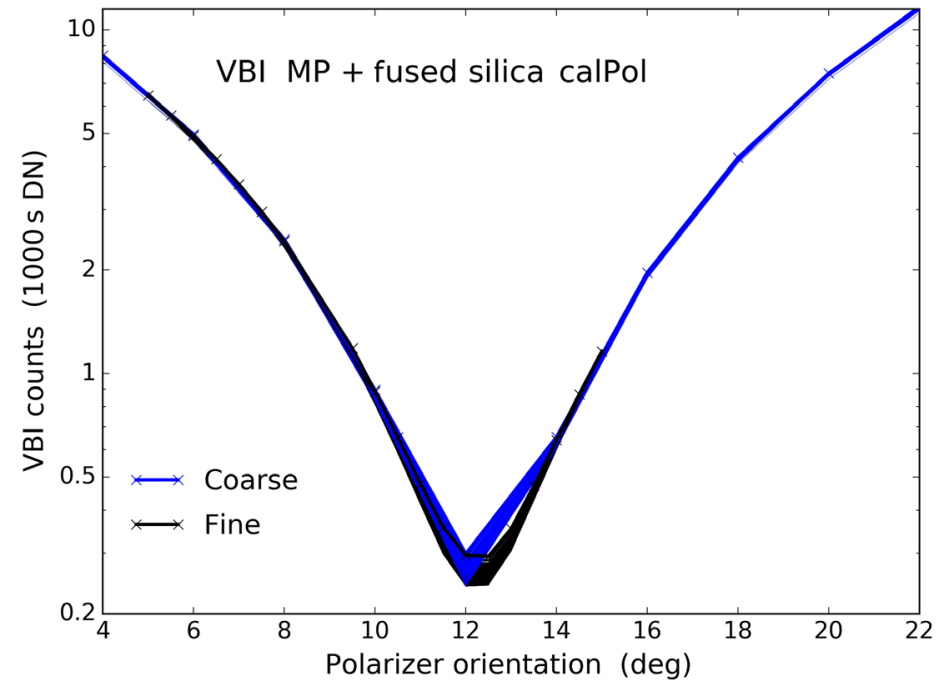

Fig. 5 The detected counts in VBI as a function of the CalPol orientation for the FS substrate CalPol1 Spare. Counts have been averaged in time over all 800 frames and then medianed spatially over all $8 \times 8$ binned pixels to provide a single number for all $4 \mathrm{k} \times 4 \mathrm{k}$ pixels for all 800 frames at each orientation. Blue shows the coarse angular sampling, and black shows more fine sampling.

camera has 16.9 million imaging pixels in $4104 \times 4128$ format. We apply $8 \times 8$ pixel binning (averaging) to each frame recorded to give 0.26 million independent results. For the fused silica polarizer (FSpol) tests, we recorded 800 frames at each orientation at $30 \mathrm{~Hz}$ cadence in bursts of 80 images.

We perform a simultaneous fit to two separate data sets collected with VBI. We sampled flux for a coarse set of angles and a more fine set of angles on the FSpol (CalPol1 Spare). Figure 5 shows the counts detected by the Balor in VBI blue as the FS CalPol is rotated down stream of the master polarizer. The VBI was set to record 800 frames at $30 \mathrm{~Hz}$ frame rate at each polarizer angle. For clarity, we average all 800 frames at each angle and then median all image pixels. The images do spatially vary by roughly $450 \mathrm{DN}$. The statistical noise properties of the images also vary spatially by more than a factor of 2 . The VBI data had a fairly constant shot noise $(\sigma)$ of $18 \mathrm{DN}$ with some patches up to $25 \mathrm{DN}$. We can also compute pixel-wise noise statistics within each 64 pixel box when doing the $8 \times 8$ spatial binning for each of the 800 frames to assure data quality. The VBI data had a spatially uniform intensity pattern with variation levels near $160 \mathrm{DN}$ but with some small but clear column structures from the Balor sensor electronics.

The fit to Malus' Law for rotating polarizers gives a three-variable equation for the detected intensity $I_{0} \cos ^{2}\left(\theta+\theta_{0}\right)+B$. Provided a wide enough angular sampling is obtained, we can fit for the peak intensity for parallel polarizers separate from the background. The background is a combination of dark/bias residuals as well as actual flux from imperfect polarizer contrast. The model fits the data to roughly $200 \mathrm{DN}$ peak to peak on a base of $8400 \mathrm{DN}$. There is clear photometric variation in the net flux at levels of $<100$ counts peak-to-peak seen as consistent frame by frame variation. Data were recorded late in the afternoon with cumulus clouds very close to the observatory. With multiple repeated data sets, this photometric issue could be reduced. However, as we show later, the fitting precision is better than $0.001 \mathrm{deg}$ with this photometric stability and other effects limiting accuracy. With these 4-ms exposures, the fits to $I_{0}$ suggest we would have expected up to 400,000 DN, well over the full well of the sensor. This background term B contains both residual dark subtraction as well as the leaked flux from imperfect polarizer contrast. For this polarizer pair in the lab, we have measured contrast ratios of at least 2000 . With this contrast, we would expect fewer than $200 \mathrm{DN}$ leaking through a beam with parallel polarizer transmission of 400,000 DN. Given that the data frames were not well dark subtracted, we simply note that we see a spatially variable background from $210 \mathrm{DN}$ to $270 \mathrm{DN}$.

The critical parameter fit from Malus' Law is the orientation offset $\left(\theta_{0}\right)$. This orientation allows us to rotate both CalPols to their proper position in absolute telescope coordinates 


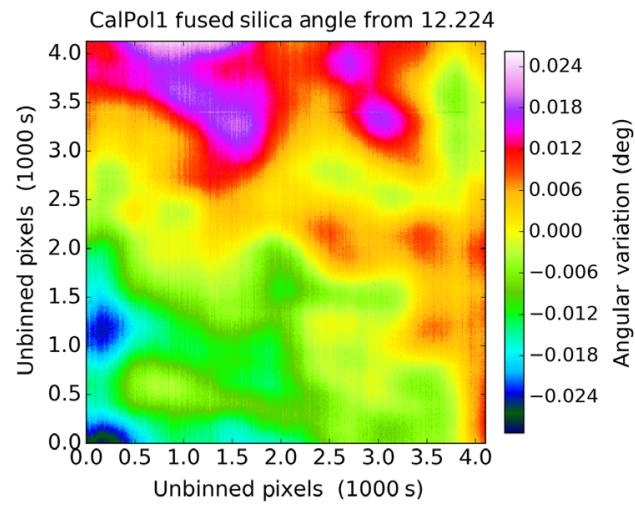

(a)

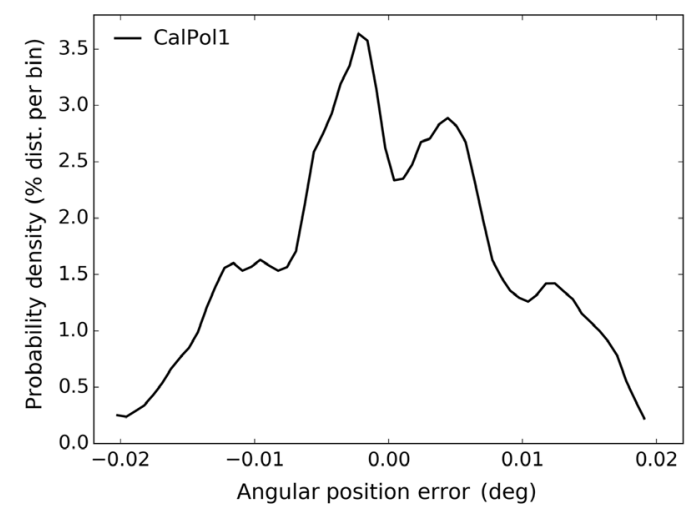

(b)

Fig. 6 (a) The best fit polarizer orientation to achieve minimum VBI counts with CalPol1 Spare on $\mathrm{a} \pm 0.025$ - deg scale. (b) The histogram of the best fit polarizer orientation about the minimum VBI counts centered about 12.224 deg.

Table 1 VBI $430 \mathrm{~nm}$ fit $\theta_{0}$.

\begin{tabular}{lccc}
\hline \hline Name & $\theta_{0}$ & $68 \%$ & $95 \%$ \\
\hline CalPol1 & $12.224 \mathrm{deg}$ & $\pm 0.009 \mathrm{deg}$ & $\pm 0.016 \mathrm{deg}$ \\
\hline \hline
\end{tabular}

referenced to the M1:M2 tilt axes. Figure 6(a) shows the spatial variation in the best fit polarizer orientation for FS. The scale runs from $\pm 0.025 \mathrm{deg}$ and contains patches that correlate with the spatial variation of transmission and contrast for these FS polarizers.

The best fit orientations do vary spatially. We derive the confidence intervals in the precision of the estimated polarizer orientations. Figure 6(b) shows a histogram of the best fit orientations about the focal plane median value. The FSpol is shown about a median of $12.224 \mathrm{deg}$ with bins running \pm 0.02 about the median. Table 1 shows the best fit orientation along with the confidence intervals for precision. We derive the cumulative distribution of errors from the histograms. These cumulative distribution functions (CDFs) show a measure of fit precision as assessed by spatial variation across the VBI sensor. We use them to define the $68 \%$ and $95 \%$ confidence without assuming Gaussian errors.

\subsection{Alignment of the Calibration Polarizers with NCSP and MasterPol}

We now compare the single VBI wavelength fit of the calibration polarization orientation with spectral data sets collected with NCSP. On Saturday, August 15, 2020, we ran scripts that coordinated bursts of five NCSP spectra as the CalPols were rotated down stream of the master polarizer. The Master Pol was set to the nominal orientation crossed with the M1:M2 tilt axis, and the CalPol was rotated down stream. The benefit of NCSP is the simultaneous recording of more than 1500 spectral pixels with continuous wavelength coverage from 380 to $1650 \mathrm{~nm}$. The complication is that the nominal NCSP optical configuration includes an analyzing polarizer in the beam which couples polarization artifacts into this measurement. The rotating CalPol does extinguish the $>99.9 \%+Q$ beam created by the master polarizer. However, rotating the CalPol rotates the linear polarization state injected into the telescope. The telescope has wavelengthdependent retardance which also changes with time as the telescope tracks the Sun. This retardance can cause the intensity passed by the NCSP analyzer to be skewed away from the nominal null between the master polarizer and CalPol. We used the Az coudé tracking mode to minimize system retardance by ensuring that the M5:M6 mirror group share a constant orientation between their respective planes of incidence with the coudé mirrors M7:NCSP. We also recorded data sets with and without the NCSP modulator in the beam. This in/out test can highlight systematic 


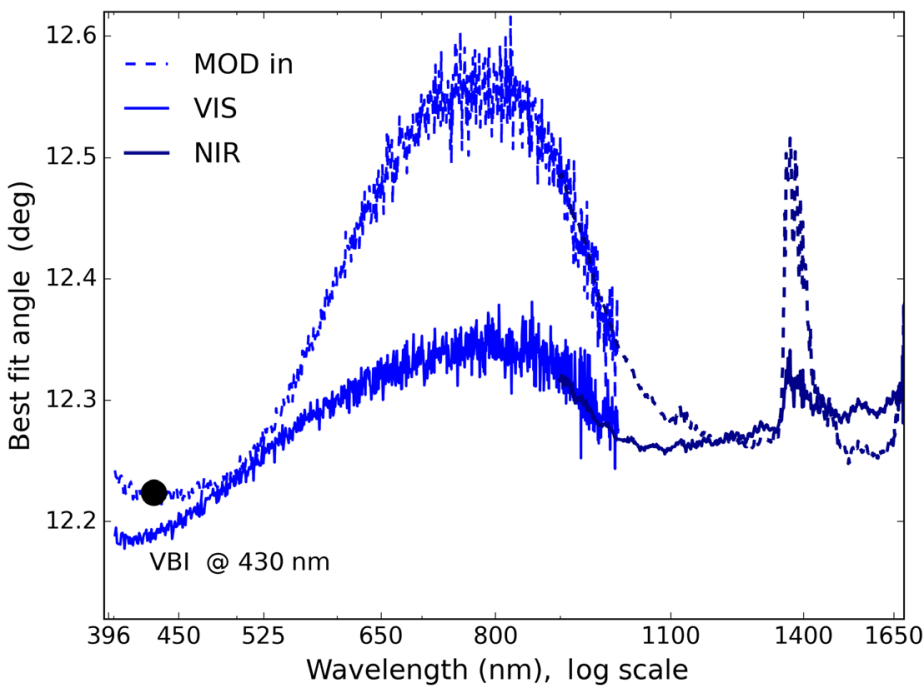

Fig. 7 The best fit angle to achieve minimum NCSP flux for the FS CalPol. Each spectral pixel is fit independently. Blue shows the VIS spectrograph while dark blue shows the NIR spectrograph. The dashed lines show the fit results when the NCSP modulator is in the beam parked at the nominal 0 deg rotational position. The single large black dot shows the best fit angle derived from the $430-\mathrm{nm}$ wavelength VBI data sets at $12.224 \mathrm{deg}$.

errors tied to the polarization response of NCSP. As VBI does not have an analyzer, the artifacts caused by system polarization are greatly reduced.

Using NCSP, we perform the same fit to Malus' Law as in VBI. We fit each spectral pixel separately but now have no spatial information as the NCSP fiber samples some particular patch of the focal plane. The NCSP data set looks very similar to Fig. 5 but with detected counts now varying from hundreds to a few thousand and with null count levels in the range of 0.1 to $20 \mathrm{DN}$. The NCSP fiber samples 2.9 arc sec of field using the 0.6 -mm-diameter fiber core and a magnification to $1.0 \mathrm{~mm}$ by the $5: 3$ focal length ratio of the lenses inside NCSP.

There is significant wavelength dependence to the best fit angle for null flux at magnitudes of up to $0.5 \mathrm{deg}$ with the modulator in the beam and $0.2 \mathrm{deg}$ with the modulator out of the beam. The wavelength dependence of this null flux angle is shown in Fig. 7. Dashed lines show the modulator in fits with the larger wavelength dependencies. The atmospheric absorption band wavelengths are significantly impacted with the 1300- to $1450-\mathrm{nm}$ bandpass. The variable atmosphere leads to photometric errors during our measurements, creating 0.3-deg systematic errors through the transparency variation. For both experiments, the coudé table angle was set to track the telescope azimuth, ensuring the M5:M6 group and M7:NCSP shared an incidence plane. The DKIST mirror coatings have zero retardance at wavelengths near 450 and $850 \mathrm{~nm}$. For the FSpol CalPoll Spare, there is agreement between the three measurements. The modulator-out measurement is within the range $12.19 \mathrm{deg}$ to $12.33 \mathrm{deg}$ for the entire band, in good agreement with the VBI measurement of $12.224 \mathrm{deg}$.

\subsection{Future Accuracy Improvements for Polarizer Orientation}

These preliminary fits show that VBI at $430 \mathrm{~nm}$ and NCSP over 390 to $1650 \mathrm{~nm}$ agree to better than $0.4 \mathrm{deg}$ for the FSpol. This initial fit is sufficient to proceed with fitting system model parameters and initial telescope mirror model database population. We anticipate running several more cross comparisons with VBI and NCSP at multiple wavelengths, pointings, and optical configurations to assess the systematic errors clearly impacting the fits of Fig. 7. We also show a perturbation analysis in Appendix B where the CalPol is assumed to be at different orientations and system model parameters are refit. For this paper and the initial DKIST commissioning process, we adopt the VBI values noting that future assessments will be required to bring this orientation below 0.1 deg orientation uncertainties. 


\section{System Model Calibration with NCSP and GOS Lamp}

We create an initial polarization model for DKIST and outline procedures for fitting a typical system model data set in this section. On March 6, 2020, we recorded several hours of NCSP data using the GOS ALS to create a preliminary lamp-based system polarization model. We collected these data months before installing or aligning the master polarizer. Without that absolute reference, we set the FS CalPol at a lower precision estimated location within \pm 10 deg of the DKIST coordinate system. As we are fitting the input Stokes vector components using a lamp which creates elliptical polarization, we simply set an offset angle between the CalPol and the $+Q$ reference frame in the analysis. Future on-Sun observations will have the polarizer set to the $+Q$ orientation, theoretically removing the need to fit for input $U$ terms. We also show here a sensitivity and perturbation analysis to demonstrate how sensitive the fitting models and errors are to the assumed CalPol orientation.

\subsection{System Calibration Data Acquisition and Diversity}

There are choices to be made for how many input states are collected, how many individual PolCal data sets are required, and at how many coudé table angle orientations when collecting a full system calibration data set. During this initial testing, we found we needed roughly $10 \mathrm{~min}$ per CS and we spent roughly $5 \mathrm{~h}$ calibrating the DKIST and NCSP system. With this duration and speed limitation, we planned to cover three telescope azimuths of 90, 180, and 270 combined with two telescope elevations of 15 and 75. We used a Dunn Solar Telescope (DST) standard procedure, setting the coudé laboratory table angle offsets to $0,90,180$, and 270 for each telescope Az/El combination (Table 2).

This grid gives 24 total polarization CSs (PolCals). Near the end of the day, we were able to acquire two extra Az/El combinations at non-standard pointings of [90 deg, $45 \mathrm{deg}$ ] and [0 deg, $104 \mathrm{deg}$ ] for a total of 26 . There were 12 total inputs representing the 10 -state sequence plus exposures at the beginning and end where we record modulated flux without any GOS calibration optics. We call these modulated flux measurements the clear exposures as GOS is clear of polarization components. We list in Table 3, the GOS polarizer (CalPol) and retarder (CalRet) orientations for our nominal 10-state sequence used to create a diverse set of Stokes vectors injected into the telescope. NCSP used six modulation states for each of these GOS input states. We implemented a double sided modulation for improved timing where we assume $180 \mathrm{deg}$ symmetry in the modulator retardance. We treat the modulator positions $0 \mathrm{deg}$ to $180 \mathrm{deg}$ as the same as $180 \mathrm{deg}$ to $360 \mathrm{deg}$. This avoided a long NCSP modulator motor move which would have taken a few seconds per step, substantially reducing duty cycle. With good optical beam centering, this assumption is valid within alignment tolerances and beam deflection.

We collected 1872 exposures for both VIS and NIR spectrographs. We used a 25-s lag time to coordinate NCSP modulation and GOS calibration motor move times. We set NCSP spectrographs to $900 \mathrm{~ms}$ exposures for 5.4 total seconds of integration and a $22 \%$ duty cycle. The VIS system was heavily filtered to avoid saturation and color balanced to achieve significant flux at

Table 2 GOS ALS + NCSP nominal data set.

\begin{tabular}{lcc}
\hline \hline Variable & $N$ & Description \\
\hline$N_{\text {mod }}$ & 6 & Number of NCSP modulation states \\
$N_{\mathrm{CS}}$ & 12 & Number of GOS calibration states \\
$N_{\mathrm{TA}}$ & 4 & Number of coudé table offset angles \\
$N_{\mathrm{Az}}$ & 3 & Telescope mount azimuth angles \\
$N_{\mathrm{El}}$ & 2 & Telescope mount elevation angles \\
$N_{\mathrm{PC}}$ & 72 & Spectra per PolCal $=N_{\text {mod }} * N_{\mathrm{CS}}$ \\
\hline \hline
\end{tabular}


Table 3 Sequence 10.

\begin{tabular}{lc}
\hline \hline CalPol & CalRet \\
\hline Out & Out \\
0 & Out \\
60 & Out \\
120 & Out \\
0 & 0 \\
0 & 60 \\
0 & 120 \\
45 & 30 \\
45 & 90 \\
45 & 150 \\
45 & 0 \\
Out & Out \\
\hline \hline
\end{tabular}

$393 \mathrm{~nm}$ wavelength. The VIS system used Hoya LB-120, LB-100, LB-40, and ND-10-reflective all stacked together. Scaling the lab characterization estimates of 8 electrons per count per co-add, we get roughly 400 electrons per count using 50 co-adds and an $18 \mathrm{~ms}$ integration time. The 18-bit encoder was linear through at least 150k counts and we derive a peak SNR near 7500. The NIR system gain is roughly $75 \times$ higher at 600 electrons per count per co-add. We used 36 co-adds and a 25 -ms integration. The peak SNR level is near 25,000.

\subsection{System Calibration: Alignment and Optical Challenges}

There are a number of limitations to this first preliminary data set. This system model will be improved in the early operations phase of DKIST as optical and mechanical alignments are improved and the facility instruments get installed.

For our 2020 data sets presented in this paper, the beam delivered to the coudé laboratory was estimated to only be stabilized to \pm 9.7 arc sec field angle (out of the 5 -arc min field). We did not have access to active Az-El-TA-dependent beam compensation using the look up tables for pointing the active mirrors M2, M3, and M6. Our NCSP optical feed presently precludes using the WFC imaging system simultaneously. The estimates for the optical alignment drifts with pointing derived from the WFC imager were \pm 1.6 arc sec on the coudé lab rotation axis, \pm 1.8 arc sec on the azimuth axis, and \pm 6.3 arc sec on the altitude axis in effective field angle at the time of our 2020 data sets. This is several times larger than the 2.9-arc sec NCSP fiber diameter though small relative to flat field variations expected for the DKIST calibration lamp.

This beam pointing instability causes several potential issues for this preliminary data. First, the CalRet and CalPol are not sampled on-axis with a constant beam footprint during all rotations of both optics. Different telescope pointings sample different patches of the optic and produce state-dependent system inputs (see HS18b ${ }^{66}$ Sections 6 and 7, and $\mathrm{H} 20^{80}$ ). Thus we are limited by spatial non-uniformity of both polarizer and retarder. Polarizer spatial behavior we have measured is fairly benign with measured transmission spatial variations of a few percent being the more dominant error source. The orientation of polarization is stable across the aperture to better than \pm 0.05 deg with wavelength dependence of roughly half this value. Contrast does also vary spatially by factors of several, but contrast remains high in all DKIST CalPols.

With the beam sampled near \pm 10 arc sec field angle, we illuminate different patches of the mirrors coupling in coating non-uniformity. We also have variable incidence angle as outlined in 
$\mathrm{HS} 17^{61}$ accounting for the $20 \times$ pupil demagnification. In addition, there is a slight decenter of $2.5 \mathrm{~mm}$ for the retarders and polarizers as currently mounted with respect to the telescope gut ray. Flexure adds another a fraction of a millimeter spatial variation as gravity deforms the calibration optic mounting system through the day. The beam footprint is 26.6-mm-diameter on the retarders. This decenter of roughly $10 \%$ footprint causes some mild variation of the retarder properties, even for the on-axis beam, increasing the fitting error (see HS18b ${ }^{66}$ ). This decenter also impacts polarizer errors through spatial transmission variation. Similarly, we have not yet implemented any M2 piston to compensate for focus changes caused by the varying thickness and refractive index of the various crystal retarders and polarizers. There is thus a very small, assumed negligible, difference in focus depending on which calibration optics are inserted in the beam.

The GOS lamp fluctuates in time, impacting the photometric stability. We compensate for the time dependence over several minute timescales through application of a linear trend correction. However, there is some higher speed flicker that remains uncorrected. Initial laboratory stability estimates showed $0.3 \%$ photometric variations at timescales spanning 0.1 to $4 \mathrm{~Hz}$ with a very flat power spectrum. These fluctuations, however, were very stable in time meaning that temporal averaging of many frames very successfully averages out this higher speed flicker. We measured lamp photometric drifts of $<0.5 \%$ per hour, roughly consistent with NCSP stability measurements we present below during a day of lamp calibration. Future testing is planned at $200 \mathrm{~Hz}$ in the coudé laboratory to assess this drift.

The NCSP was set to assume the 0-deg to 180-deg orientation modulation states were identical to the 180- to 360-deg states. This causes several known issues in modulation. For NCSP, the largest issue is coupling in the beam deflection through fiber transmission changes as well as possible retarder variation from decentered and tilted mounting.

We also have only a preliminary mechanical alignment of the retarder rotary stages against the DKIST beam using CMM and laser tracker tools to achieve 6 arc min tilt alignment of the rotary stages with at least double this expected from mechanical tolerances between the optic surfaces and the rotary stage. An optical reflection technique to reduce tilt error of the retarders and polarizers below 1 arc min is planned but has yet to be successfully performed. For now, we assume that the retarder tilts are $< \pm 0.3 \mathrm{deg}$, can be estimated using the angular sensitivities in Sueoka 2016, ${ }^{15}$ and produce on-axis errors similar to the off-axis field-dependent retarder spatial variation terms we published in HS18b. ${ }^{66}$ These terms are small and expect to be improved in near future.

The GOS CalRets each have their own beam deflection which causes angle dependent misalignments. There are two oiled retarders: one called the ViSP super-achromatic retarder (SAR) and another called the CN SAR (see HS18b ${ }^{66}$ for some details). The beam deflection was estimated for both these parts through interferometric measurements to be $<10$ arc sec. For the optically contacted calibrator (OCcal), one of the crystals was deterministically fluid jet polished to 0.05 arc sec deflection flatness, and the contacted pair was measured to have 1.2 arc sec beam deflection $\left(\mathrm{H} 20^{80}\right)$. This beam deflection near a focal plane causes the beam footprints to move slightly on downstream mirrors as a function of each mirror station for each retarder rotation throughout the CS. We have measured coating uniformity on several optics and have specifications for the allowable coating variation, but this is a very small but non-zero error that can be improved through reduced deflection in optically contacted retarders.

There has not yet been an absolute orientation assessment between the coudé laboratory and the telescope mount. The tolerance between M7 and M6 incidence planes results in some coudé offset being not strictly zero (e.g., when azimuth $=180 \mathrm{deg}$, table angle $=0 \mathrm{deg}$ ). This offset has minimal impact to the fitting error, as the modulation matrix fitting accounts for any rotations. However, when comparing with our lab metrology, the assumption of shared incidence planes will impact the metrology prediction of the modulation matrix as we assume sharing a $+Q$ incidence between M6 and M7. We outline below a small but significant change in the modulation matrix fitted below that could be partly attributable to the NCSP system not optically sharing a $+Q$ incidence plane with telescope mirrors when it assumed to be aligned in software.

We performed the mirror group calibration with the CalPol misaligned with the M1:M2 tilt axis by roughly $4.2 \mathrm{deg}$. We had not performed the on-Sun master polarizer alignment and used 
a laboratory metrology estimate. We include this polarizer offset orientation in all fits, but we do introduce some $U$ terms, which requires us to fit for $U$ input variables to achieve accuracy. Future fits will be done with the polarizer aligned at least $10 \times$ better than this preliminary orientation, allowing us to force certain system model variable terms to zero, allowing a better assessment of the accuracy limitations.

Though we have several optical challenges, these preliminary fits presented here show that the metrology and the system models agree, that we can fit the system to reasonable photometric accuracy, and that the many-variable fit routines are robust and stable. We anticipate alignment improvements as DKIST begins early operations in the coming year with the opportunity to repeat many of our calibrations presented here for improved accuracy. We also will not suffer many of these issues when calibrating the facility instruments as the AO wavefront sensor system, active alignment system, context imager(s) (CI), and improved optical alignment will all assist in stabilizing the beam pointing for future calibrations.

\subsection{Transmission Functions and Intensity Detrending}

There are many transmission factors included in a system model that can be degenerate. We independently fit transmission functions for optics that are inserted and removed from the beam during a CS as these fits are well conditioned. This includes the CalPol and CalRet at the GOS. We throughout this paper abbreviate the CalPol and CalRet as either. All other transmission values for mirrors, filters, fibers, etc., are commonly combined into a single system throughput variable $I_{\text {sys }}$ that scales the total detected flux. We fit normalized Mueller matrix elements for all optics and combine the resulting transmission into a single global value fit separately for each PolCal (see Appendix D for the normalized Mueller matrix equations and terms).

The mirror groups 1:2, 3:4, and 5:6 represent two mirror throughput terms each. The combined coudé mirrors from our flux budget include 7:8:9 and then the CN path of M9a to the CSM, the $\mathrm{CN}$ focusing mirror creating the $F / 18$ beam, and then the four NCSP fold mirrors. NCSP in transmission to the visible spectrograph includes a collimating lens, modulator, analyzer ( $-Q$, transmitting one polarization state), the focusing lens, and fiber feeding the spectrograph. The spectrograph itself has slit losses, grading and mirror losses, a variable linear blocking filter, as well as variable quantum efficiency on the sensor. We use different combinations of density filters and color balancing filters to bring both VIS and NIR spectrograph count levels and exposure times into balance at speeds close to the system limitations. The system intensity variable $\left(I_{\text {sys }}\right)$ represents the brightness of the source at each wavelength as well as the transfer and detection efficiency of the entire system. In addition, we must separate the (elliptical) polarization created by the optics of the system. The $I_{\text {sys }}$ variable nominally corresponds to photoelectrons detected for unpolarized flux incident on the entire system ahead of the DKIST primary (M1) or the calibration lamp.

The modulated exposures where no CalPols or retarders are in the telescope beam are commonly called "clears." Figure 8 shows the mean clear spectrum before and after all 26 PolCals taken during the NCSP testing of the lamp. These are the $I_{\text {sys }}$ estimates fed into the system model fitting modules. We also note that we use these measurements later in a much more simple fit as the system throughput without any fitting. Note the 59.7-m optical path between GOS and NCSP can introduce substantial atmospheric absorption in the oxygen bands, water bands, etc. at 950 , 1150 , and $1400 \mathrm{~nm}$.

\subsection{Intensity Normalized Modulated Spectra}

The individual data sets are spectrally smooth and contain obvious clocking misalignment spectral oscillations caused by the imperfect cancelation of one crystal net retardance with the other in a compound retarder pair $\left(\right.$ see $\mathrm{H} 20^{80}$ ). The spectra agree well with orthogonal analyzers between VIS and NIR spectrographs. We show the 72 spectra for the first PolCal in Fig. 9(a). Blue shows the VIS spectrograph data normalized by the mean of the modulated clear exposures. Green shows the NIR normalized data set. In Fig. 9(b), we show the 26 different PolCals where each color represents a particular modulation state. The first 12 PolCals were taken with the telescope at elevation $15 \mathrm{deg}$, whereas the second group of 12 PolCals had the telescope elevation at $75 \mathrm{deg}$.

J. Astron. Telesc. Instrum. Syst. $\quad$ 018004-16 Jan-Mar 2021 • Vol. 7(1) 


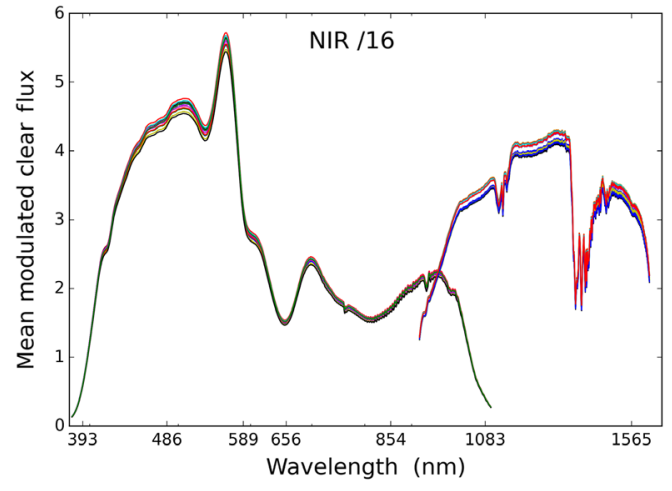

(a)

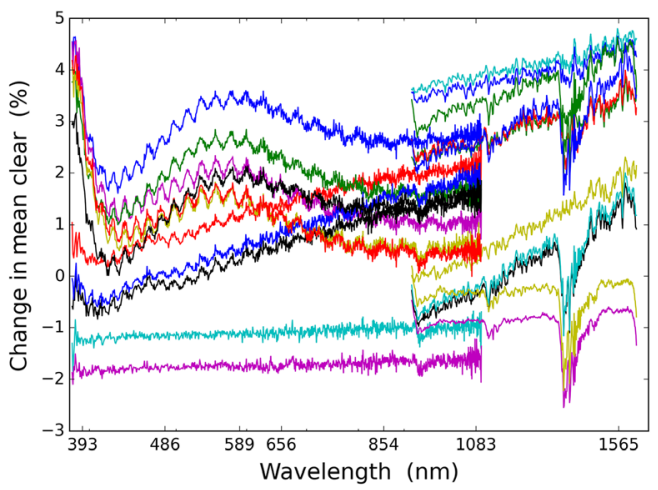

(b)

Fig. 8 (a) The 26 NCSP lamp $I_{\text {sys }}$ estimates computed as the mean of both sets of modulated spectra without any CalPols or retarders inserted near Gregorian focus (commonly called clears). The $Y$ axis is millions of photoelectrons. The NIR spectra have been divided by 16 for convenient plotting range. (b) The change in time from the first $I_{\text {sys }}$ estimate computed as the difference divided by the sum in units of percent. The beam alignment issues mentioned above couple in the flat field (spatial variation of intensity at the focal plane) as well as alignment and fiber injection issues through the changing field angle ( \pm 30 arc sec). Variation in the atmospheric absorption bands is also present and obvious near $1400 \mathrm{~nm}$ wavelength.

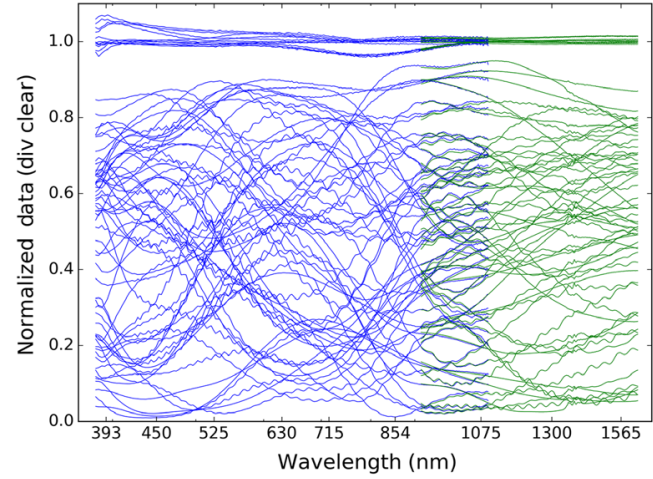

(a)

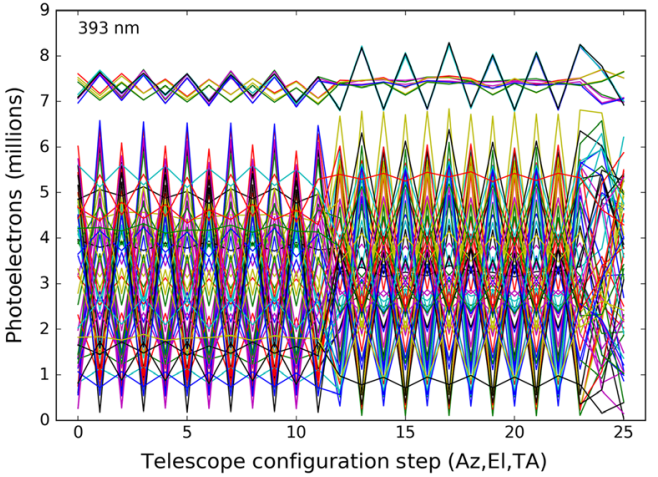

(b)

Fig. 9 (a) The mean-clear-normalized intensity ratios for the first of 26 NCSP lamp PolCals. The visible spectrograph data are shown in blue, whereas the near-infrared spectrograph data are shown in green. There is good agreement in the overlap wavelength range of 900 to $1080 \mathrm{~nm}$. (b) The detected photoelectron levels for $393 \mathrm{~nm}$ wavelength as a function of all 26 telescope optical configurations. Each color represents a different modulation state (6) and CS step (12) for the 26 different PolCals. The first 12 data sets were at an elevation of 15 deg with the second 12 data sets at an elevation of $75 \mathrm{deg}$. The last two steps are at intermediate telescope pointings. The upper curves show a change in polarization magnitude and orientation. The colorful pattern repeats for each block of 12 configurations showing that rotating the azimuth and coudé table angle produces the expected stable symmetries. The modulated clear exposures are near 7 to 8 million photoelectrons. The polarization of the input beam causes the modulation of these clear exposures at count levels between 7 and $8 \mathrm{M}$. The steps with the polarizer and retarder in the beam span nearly the full range of brightness levels with a dynamic range of at least 1000.

The last two PolCals were at intermediate telescope Az, El pointings. We note the data were collected with azimuths of $90 \mathrm{deg}, 180 \mathrm{deg}, 270 \mathrm{deg}$ with the coudé table offset in integer multiples of $90 \mathrm{deg}$. This gives the symmetry to the individual configuration steps. There is substantial symmetry with $180 \mathrm{deg}$ rotations of optics in polarimetry, and this behavior is reproduced in Fig. 9(b). 


\subsection{Temporal Detrending Within a PolCal}

There is an optional data analysis step to perform a linear flux trend correction over the duration of a PolCal sequence. We anticipate this option will always be used as the benefits are significant whether tracking the Sun or using the calibration lamp. The two modulated clear flux measurements at the beginning and end of each CS are averaged over the modulation cycle. The temporal instability of the lamp brightness or atmospheric transparency variation is corrected to first order with a linear detrend. The mean of all modulated clear exposures is recorded before and after the initial 10-state CS. The linear slope is derived between these before and after means. The flux at each time within a CS is adjusted according to this linear slope correction. The intensity correction is applied within each GOS optic step, but not within each modulation state at this time.

The M1:M2 mirror group does partially polarize the incoming solar flux. The telescope does contain time-variable cross talk when tracking the Sun causing some small variation in the Stokes vector at the modulator for these two clear measurements separated by roughly a few minutes up to $15 \mathrm{~min}$. For the lamp measurements, the mount and coudé table are parked and stable. However, the atmospheric transparency changes between two modulated clear measurements should be much larger than the flux levels from intrinsic telescope polarization changes over the few minute duration of a typical sequence. The mirror retardance and diattenuation change when tracking the Sun. Only for extreme near-zenith pointings would there be more than a few degrees Az/El axis rotation over a 7-min data set. The average of the modulated clear measurements should represent a high-accuracy measure of photometric variability over the few minutes of a PolCal. We note that our fitting routines update the telescope geometry on a per GOS calibration state basis, which would account for telescope changes at faster than 30-s cadence. We know this error exists and can estimate it for any particular wavelength using the mirror group model, CS duration and pointing should photometric errors ever show residual linear trends.

The telescope changes in azimuth, elevation, and table angle occur at every step of the CSs and are updated in the fitting routines at nominal cadences faster than $30 \mathrm{~s}$. The geometry changes are thus not tracked on timescales shorter than the modulation cycle, which allows calibration while using Az/El tracking for all but the highest solar elevations where the coudé table rotation rates exceed the table speed limits. The thermal perturbations of the retarder are corrected on timescales longer than the several minutes of a PolCal.

Figure 10 shows the variation in measured brightness with time and wavelength derived from the mean of the modulated clear exposures. Figure 10(a) shows the normalized difference spectra

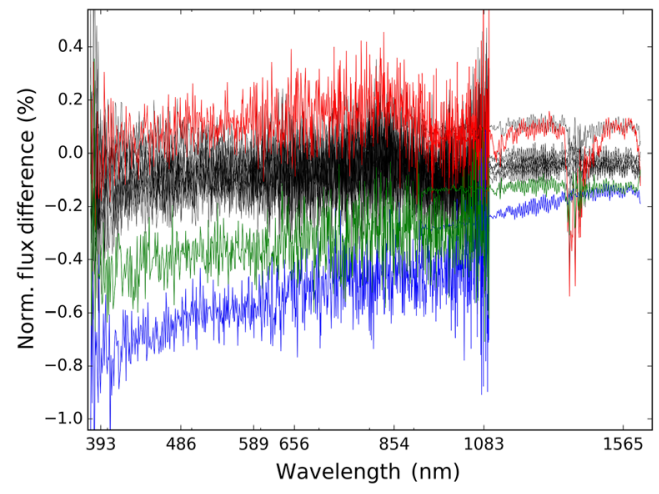

(a)

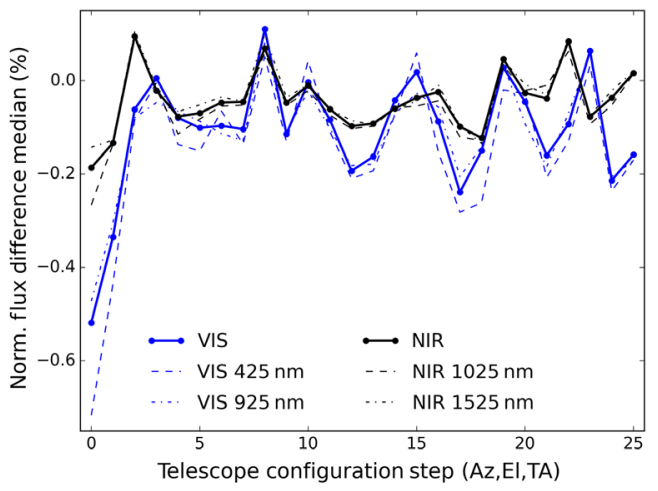

(b)

Fig. 10 The wavelength and time dependence of the mean of the modulated clear intensity spectra. (a) The normalized difference in percent between the mean of the clear exposures before and after the 10-state CS. Three large outliers corresponding to PolCals 0,1 , and 8 are shown as blue, green, and red. (b) The median of all wavelengths as dots and the thicker solid line for each spectrograph (VIS and NIR) now plotted for each PoICal as the telescope configuration changes. The first two PolCals of the day showed the largest brightness changes of $-0.5 \%$ VIS and $-0.2 \%$ NIR. The dashed lines show $50 \mathrm{~nm}$ of bandpass about each central wavelength in the legend. Shorter wavelengths vary more than longer wavelengths for both spectrographs. 
in percent between the mean of the modulated clear exposures at the start of the sequence (exposures 0 to 5) and those at the end of the sequence (exposures 66 to 71). The visible spectrograph has shot-noise limits near $0.1 \%$ RMS with some moderate wavelength dependence. A few spectra stand out as having changed by more than $0.5 \%$ brightness at most wavelengths during the duration of the 72 exposure PolCal. We highlighted three large outliers for PolCals 0,1 , and 8 as blue, green, and red, respectively, in Fig. 10(a). The near-infrared spectrograph has much higher signal-to-noise ratios and saw correspondingly smaller normalized differences in lamp brightness, though there are still a few obviously different spectra. This is a reasonable proxy for lamp brightness as the telescope mount was parked during the exposures. The strong wavelength dependence for the blue curve from $-0.8 \%$ lamp brightness change at $400 \mathrm{~nm}$ wavelength falling to $-0.2 \%$ change at $1500 \mathrm{~nm}$ wavelength suggests a temperature change as well as a lamp power dissipation change. We also can see variation in the atmospheric absorption bands near 1350 to $1450 \mathrm{~nm}$ wavelength.

Figure 10(b) shows the gradient between beginning and end mean clear exposures for select wavelengths for each of the 26 different CSs recorded during the data set. The first two data sets show a stronger gradient of $-0.5 \%$ for the VIS spectrograph and $-0.2 \%$ for the NIR spectrograph. The dashed lines highlight different bandpasses with shorter wavelengths varying more than longer wavelengths generally for all exposures but not in all cases. The lamp output power changes impact both brightness and effective color.

\subsection{Noise Model, Merit Function, and Fitting Method}

$$
\begin{gathered}
\sigma_{\text {shot }}^{2}=I_{0}=g * \mathrm{DN}, \\
\mathrm{SNR}=\mathrm{SNR}_{0} * \sqrt{\frac{I}{I_{0}}} .
\end{gathered}
$$

The model for statistical noise in NCSP spectra consists of two components. The shot noise computed from photon statistics has the variance equal to the mean photon count level $\left(I_{0}\right)$ as in Eq. (9). The units of $I$ must be in photoelectrons so we convert from measured count values (DN) using the gain $(g)$ in photoelectrons per DN measured during system characterization. We use the nominal signal-to-noise ratio $\left(\mathrm{SNR}_{0}\right)$ at some detector flux value $\left(I_{0}\right)$ to account for the system gain and scale the statistical noise to the detected flux following $\sqrt{n}$ as in Eq. (10). We include the read noise component at zero exposure level as $\sigma_{\text {read }}$ :

$$
\varepsilon^{2}=\sum_{i=1}^{N_{\mathrm{spc}}}\left[\frac{I_{\text {model }_{i}}-I_{\mathrm{data}_{i}}}{\sqrt{I_{\mathrm{data}_{i}}+\sigma_{\mathrm{read}_{i}}^{2}}}\right]^{2} .
$$

We compute the difference between the data and model in units of statistical significance using the shot-noise and read-noise appropriate to each spectral pixel in each measurement. We divide the difference between data and model intensities by the RSS of the shot-noise and read-noise terms. As the variance is the mean count level after gain correction per Eq. (9), we use the measured data $\left(I_{\text {data }}\right)$ for the shot-noise term $\left(\sigma_{\text {shot }}^{2}\right)$. The read-noise levels are also added from system characterization. Using the statistical significance, we account for the large dynamic range in relative count levels seen above in Figs. 8 and 9 as the detected flux range varies by more than three orders of magnitude. We show this error metric in Eq. (11). We note that the subscript $i$ denotes the spectral pixel in NCSP. We use a wavelength-independent read noise as both VIS and NIR systems have roughly constant noise values for each pixel across the sensors $\left(\sigma_{\text {read }_{i}}=\sigma_{\text {read }}\right)$ however for other systems and for DKIST instruments, a pixel-dependent read noise is easily implemented per Eq. (11). 


\section{System Model Fitting Complexity: 17, 36, 62, and 137 Variables}

We outline a physical motivation for including or ignoring the many variables in a telescope and instrument system model in this section. Most variables are assumed to have negligible influence on the optical path. For instance, many simplifications are possible to the 16 element Mueller matrices for the 10 to 40 optics between the atmosphere and a DKIST instrument sensor. A very common assumption is to model a group of mirrors sharing incidence planes with tight tolerances as just three variables. Powered beams introduce depolarization through an average over many incidence angles, but these terms are small and we ignore the nine depolarization variables in the Mueller matrix for now. ${ }^{93-102}$ We estimated the DKIST M1:M2 mirror depolarization to be entirely diagonal depolarization at amplitudes below 0.002 in HS17. ${ }^{61}$ Mirror tilt misalignments can introduce additional retardance and diattenuation variables though we ignore these four variables. As an example, we demonstrate in this section that we can reasonably group 10 mirrors with 160 independent Mueller matrix terms into just 3 physical variables of transmission, $Q$ diattenuation, and $U V$ retardance. Retarders can have time-dependent properties through thermal changes (see HS18a ${ }^{63}$ and $\mathrm{H}_{2} 0^{80}$ ) but an assessment is required to show if including this variation improves the model. We show in this section some examples of system models with increasing complexity to give a physical basis to variables included. We demonstrate how including particular variables improves the photometric fitting error.

We developed two separate software packages with options to fit a range of variables with increasing complexity. This ensures agreement between multiple independent software packages and gives opportunity to identify any issues with the different approaches. We show here fits of progressively more complexity where we include physical phenomena allowing us to compare fit results with metrology of various DKIST optical components. We list the various model variables in Table 4. The first column describes the variables. The second column shows specific number of variables used when fitting a system model to our 26 PolCal lamp data set. We have the option to ignore the polarization caused by optics upstream of the GOS. We can include constrained fits for just the input Stokes $Q$ in the plane of the M1:M2 mirror tilt axis $\left(Q_{\text {in }}\right)$. We also can include fully elliptical fits $\left(Q_{\text {in }}, U_{\text {in }}\right.$, and $\left.V_{\text {in }}\right)$. This is listed in the first row of Table 4 as 0,1 , or 3 possible variables. In all of our models, we fit the transmission of the CalPol and the transmission of the CalRet. However, we have the option to fit this transmission function for each of the 26 PolCal's separately to assess if there is any time or temperature dependent changes to transmission. This is listed in the second and third row of Table 4 as 1 or 26 possible variables. We always fit the diattenuation and retardance for two groups of DKIST mirrors as we assessed the mirror retardance is one of the most dominant terms. These variables are listed in the fourth and fifth rows of Table 4. The three elliptical CalRet parameters can be fit independently for each of

Table 4 Assessed variables in 26 PolCal fitting.

\begin{tabular}{lc}
\hline \hline Variable description & Number \\
\hline Stokes inputs at GOS $\left(Q_{\text {in }} U_{\text {in }} V_{\text {in }}\right)$ & 0,1 , or 3 \\
Transmission CalPol: $t_{\text {pol }}$ & 1 or 26 \\
Transmission CalRet: $t_{\text {ret }}$ & 1 or 26 \\
DKIST mirror group 3:4 $\left(\tau_{34}, \Delta_{34}\right)$ & 2 \\
DKIST mirror group $5: 6\left(\tau_{56}, \Delta_{56}\right)$ & 2 \\
CalRet elliptical retardance & 3 or 78 \\
NCSP modulation matrix & 24 or 5 \\
NCSP mirror group $\left(\tau_{\text {ncsp }}, \Delta_{\text {ncsp }}\right)$ & 2 or 0 \\
NCSP mod. elliptical ret: ER $R_{\text {ncsp }}$ & 3 or 0 \\
Normalized system throughput: $l_{\text {sys }}$ & 26 or 0 \\
\hline \hline
\end{tabular}


Table 5 Fit scenarios.

\begin{tabular}{lccc}
\hline \hline Model & ER & $I_{\text {sys }}$ & Mod \\
\hline Vars & 3 & 0 & 5 \\
Vars & 3 & 0 & 24 \\
Vars & 3 & 26 & 24 \\
Vars & 78 & 26 & 24 \\
\hline \hline
\end{tabular}

the 26 PolCals for 78 total variables. They can also be restricted and fit globally assuming constancy throughout the day for 3 total variables. This is sixth row of Table 4 . We note that some prior telescope calibration efforts have recorded very long CSs with many $10 \mathrm{~s}$ of input states with an allowance for linear temporal variation of retardance within each single CS. We also have implemented this option but for this paper ignore yet another 78 variables for the CalRet.

The modulation matrix can be constrained in a variety of ways. The seventh row of Table 4 lists the 24 free variables of an unconstrained modulation matrix of 4 Stokes parameters by 6 modulation states. The constrained option we demonstrate here is to group the 10 NCSP mirrors and only fit a $Q$ diattenuation and a $U V$ retardance along with the three elliptical retardance parameters for the NCSP modulator. The transmissions of all these mirrors are degenerated with the system throughput variables, are normalized, and combined. We also note the modulation matrix has several techniques for enforcing normalization, with some examples in Appendix D.3. Thus we list eighth and ninth rows of Table 4 the physically constrained models for 2 mirror polarization terms and 3 elliptical retardance terms.

We can assume stability of the unpolarized input beam (lamp or Sun) for the initial fitting process. The $26 \mathrm{PolCal}$ data sets after normalization by the modulated clear exposures can either be given a separate free variable ( 26 total) or we can ignore these terms (shown later to be small) to reduce the model complexity significantly. This is shown as the last row of Table 4

We choose to outline the progressively more complex variable scenarios using 17, 36, 62, and 137 variables by number and type in Table 5 . Our first software analysis package fits the more simple models with a current limit up to 62 variables (with 26 PolCal inputs). The second software package is the official DKIST data analysis pipeline anticipated for use with the suite of instruments. This package can accommodate many options with several hundred possible variables. We show the calibrator elliptical retardance (ER) variables in the second column of Table 5. We show the system intensity variables in the third column. We show the modulation matrix variables (Mod) in the fourth column of Table 5.

In our first scenario with 17 total variables, we make the assumption to fit the 24 independent modulation matrix elements as a grouping of mirrors sharing incidence planes ( 2 variables) followed by a rotating elliptical retarder ( 3 variables). In the second 36 variable scenario, we relax the 5 physically constrained variables and fit all 24 elements of the modulation matrix freely. Both 17 and 36 variable scenarios ignore the difference between the mean of the modulated clear exposures and the actual unpolarized input flux. In the 62 variable scenario, we add 26 independent $I_{\text {sys }}$ variables. This allows the incoming Stokes vector intensity to vary from the mean modulated clear value which normalizes the data to $\sim 1$. The $I_{\text {sys }}$ variables are noted in the third column of Table 5. The official DKIST software modules are then used in the 137-variable scenario to include the fit to independent CalRet models for every CS (PolCal) anticipating thermal perturbations through the day. This gives rise to 26 different elliptical CalRet fits for each PolCal giving 78 independent variables, adding 75 variables to the fit as noted in the second column (ER) of Table 5 .

\subsection{Photometric Errors: Comparing 17, 36, 62, and 137 Variable Models}

The best fit model should reproduce the measured flux with statistically similar errors for all individual data sets without any outliers. We assess the photometric errors for their statistical properties. We ensure that all PolCal sequences, GOS states, and wavelengths have similar error 


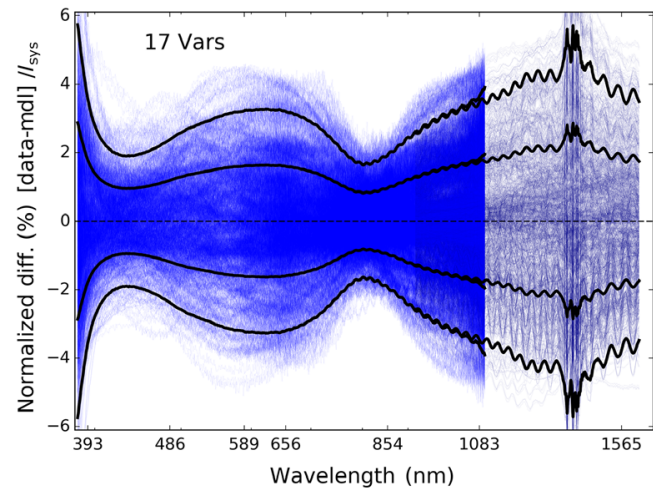

(a)

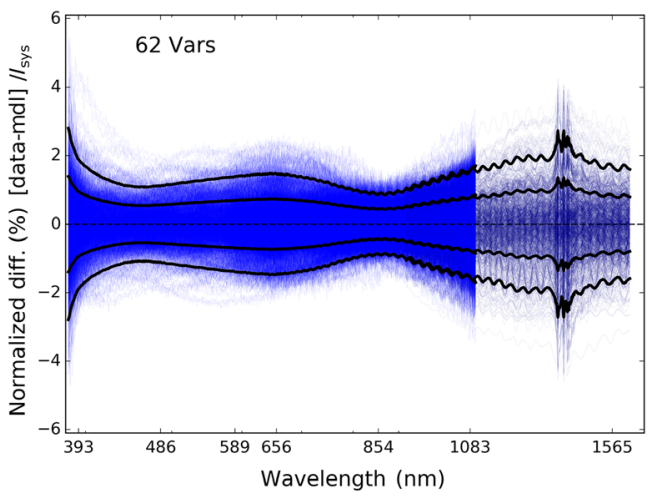

(b)

Fig. 11 (a), (b) The normalized residual photometric differences between the model and data in units of \% flux. The differences are computed as data-the best fit model after both spectrograph data sets have been divided by the $I_{\text {sys }}$ estimate as the mean of the modulated clear exposures. (a) The 17-variable fit normalized errors and (b) the 62-variable model fit normalized errors. Both models used the CalPol at 87.224 deg. The thick black lines show $\pm 1 \sigma$ and $\pm 2 \sigma$ errors.

behavior and are being fit within error limits. Our shot- and read-noise limits are far below several other error sources thus we are limited by systematic errors. We compare the 17-variable model and 62-variable model photometric fitting errors in Fig. 11. Figure 11(a) shows the 17variable fitting errors, whereas Fig. 11(b) shows the 62-variable model fitting errors. Similar results are seen for 36 and 137 variable models with slightly different behavior. At each wavelength we compute the standard deviation of all 1872 photometric residual errors for each model. The thick solid lines of Fig. 11 show the $\pm 1 \sigma$ and $\pm 2 \sigma$ error spectra. We see a significant increase in photometric residual errors in the atmospheric absorption band near 1300 to $1450 \mathrm{~nm}$ wavelength.

We also performed a simple perturbation analysis using the official DKIST software modules and the 137 system variable scenario. The best fit CalPol1 Spare orientation of 87.224 deg was perturbed by $+0.5 \mathrm{deg}$ and $+1 \mathrm{deg}$ to assess the change in wavelength-dependent photometric errors as well as the coupled perturbation of all system model variables. We detail more of this perturbation analysis in Appendix B. This perturbation shows that the photometric errors are stable without significant change when the CalPol is rotated by up to $1 \mathrm{deg}$, well above our fitting errors. We show the mean photometric $1 \sigma$ errors averaged across the 393- to 1083-nm wavelength bandpass in Table 6 . We show the nominal four models with the polarizer oriented at $87.224 \mathrm{deg}$ as the top section. The perturbed polarizer orientation models are shown in the bottom two rows.

In Fig. 12, we show the $1 \sigma$ error spectra for all six model cases run in Table 6 . The 17-variable model with the blue line has $\sigma$ above $1 \%$ with a significant wavelength trend. The bandpass average is near $1.3 \%$. Coupling of retarder clocking oscillations and the 1400-nm atmospheric

Table $61 \sigma$ Photometric error.

\begin{tabular}{lc}
\hline \hline System model & $1-\sigma(\%)$ \\
\hline 17 variables & 1.33 \\
36 variables & 0.77 \\
62 variables & 0.61 \\
137 vars, CP $87.2 \mathrm{deg}$ & 0.60 \\
137 vars, CP $87.7 \mathrm{deg}$ & 0.58 \\
137 vars, CP $88.2 \mathrm{deg}$ & 0.57 \\
\hline \hline
\end{tabular}




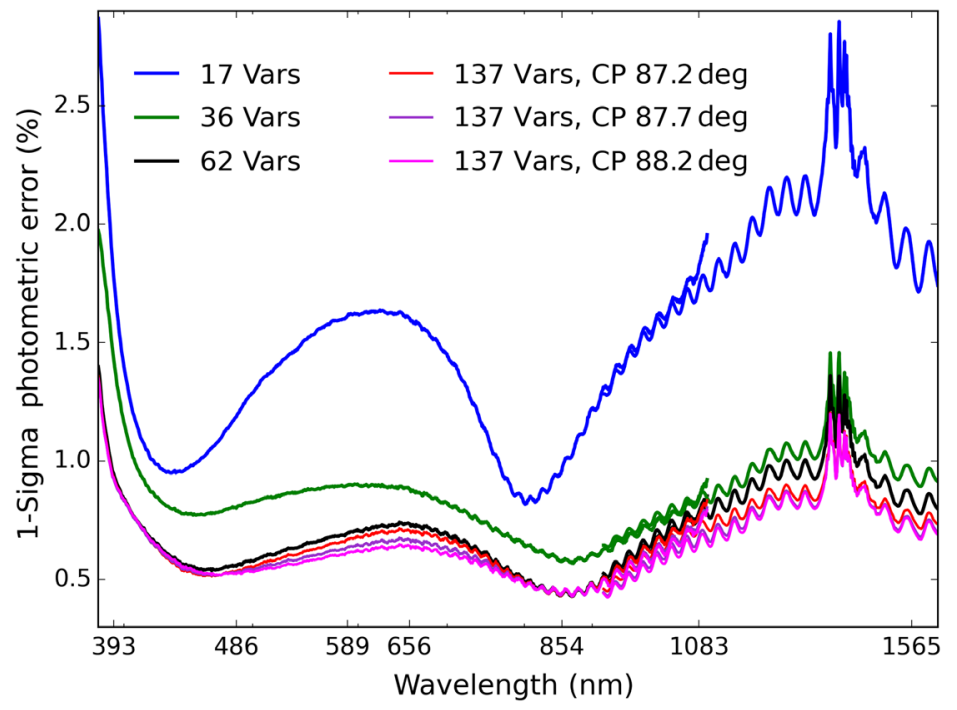

Fig. 12 The $1 \sigma$ normalized photometric errors for the 1872 spectra at each wavelength for the different variable model fits. The blue, green, and red curves show the 17, 36, and 62 variable models, respectively. The three different 137 variable models are shown in red, purple, and magenta with all three lying very close to the 62-variable model curve. Red shows the nominal 87.2 deg CalPol1 Spare orientation denoted as CP in the legend with the other colors representing perturbations away from the best fit polarizer orientation per Table 6.

band are significant. When adding 17 modulation variables to create the 36 -variable fit, the normalized photometric errors reduce strongly to $0.8 \%$ and the wavelength dependence also substantially reduces. The additional 26 variables from fitting the unpolarized input further reduce the error to $0.60 \%$ and further reduce the wavelength dependence. The red curve shows the nominal 137 -variable system model photometric errors very close to the 62 -variable model errors. These additional 75 variables corresponding to retarder temporal variation do not substantially reduce the photometric error. Per Table 6 , we only see a $0.01 \%$ change. The magenta and purple curves correspond to perturbing the CalPol orientation. There is hardly any spectral change in errors, and the bandpass averaged error from Table 6 only changes by another $0.02 \%$ or $0.03 \%$.

The photometric residual errors reasonably obey Gaussian statistics though they are a combined system response to lamp temporal variation, alignment errors, and other optical instabilities. We derived CDFs of the errors to assess that they are smooth continuous functions. After accounting for the spectrally variable $\sigma$, the photometric errors do seem to follow a Gaussian distribution with some few small outliers at the outer $3 \sigma$ limits at $0.3 \%$ of the distribution ( $1 \%$ to 99.7\%). This Gaussian behavior was seen in all models with 36, 62, and 137 variables.

\subsection{NCSP System and 17 Variable Constraints: 5 Modulation Variables}

The 17-variable system model heavily constrains the NCSP coudé optics for modulation to include only the 5 variables per Tables 4 and 5 . We do this heavy constraint to assess the choice to initialize the modulation matrix variables either as a direct solve using measured fluxes or to force a physical model prediction. We show here that the modulation matrix fitting results are stable without requiring significant initialization in the more complex scenarios. The 10 mirrors from M7 through the NCSP focus become grouped. We created a mirror-coating model for all 10 mirrors. Some of our coating models mismatch measurements by more than 10 deg retardance at wavelengths shorter than $450 \mathrm{~nm}$. However, for the purposes of establishing a baseline model for 10 grouped mirrors, we believe that the model is good enough to establish the modulation matrix fits as reasonable and physical (see Appendix $\mathrm{C}$ for more details on the NCSP mirror modeling and Appendix C.8 for the modulation matrix). Figure 13(b) shows the diattenuation of the combined coudé lab optics ahead of the NCSP modulator in the local $Q$ incidence plane. Figure 13(a) shows the retardance in the local $U V$ plane. The initial metrology estimates used as initial values 


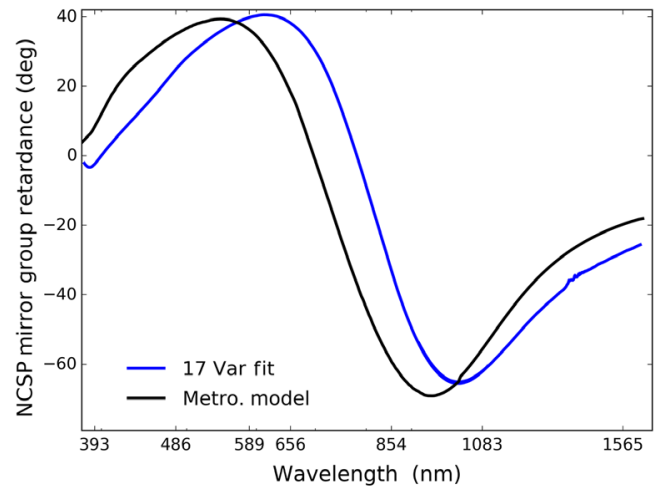

(a)

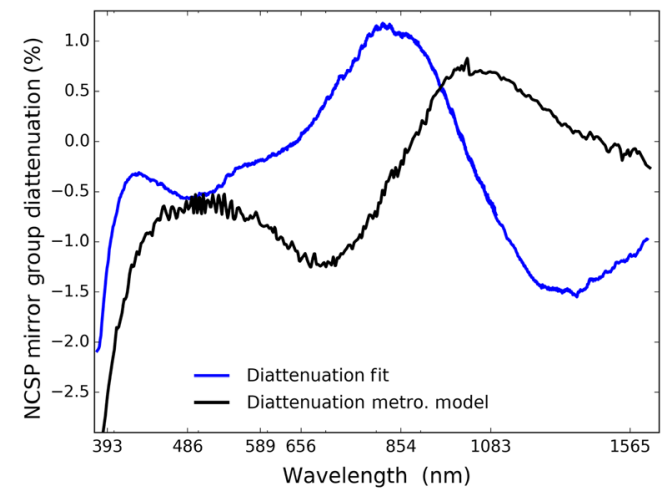

(b)

Fig. 13 The 10 mirror group (a) retardance metrology model and fit and (b) diattenuation metrology model and fits. The mirrors in the coudé lab from M7 through the last NCSP fold were combined. Black curves show models detailed in Appendix C. Blue curves show the fit results to the DKIST system calibration with 17 total model variables.

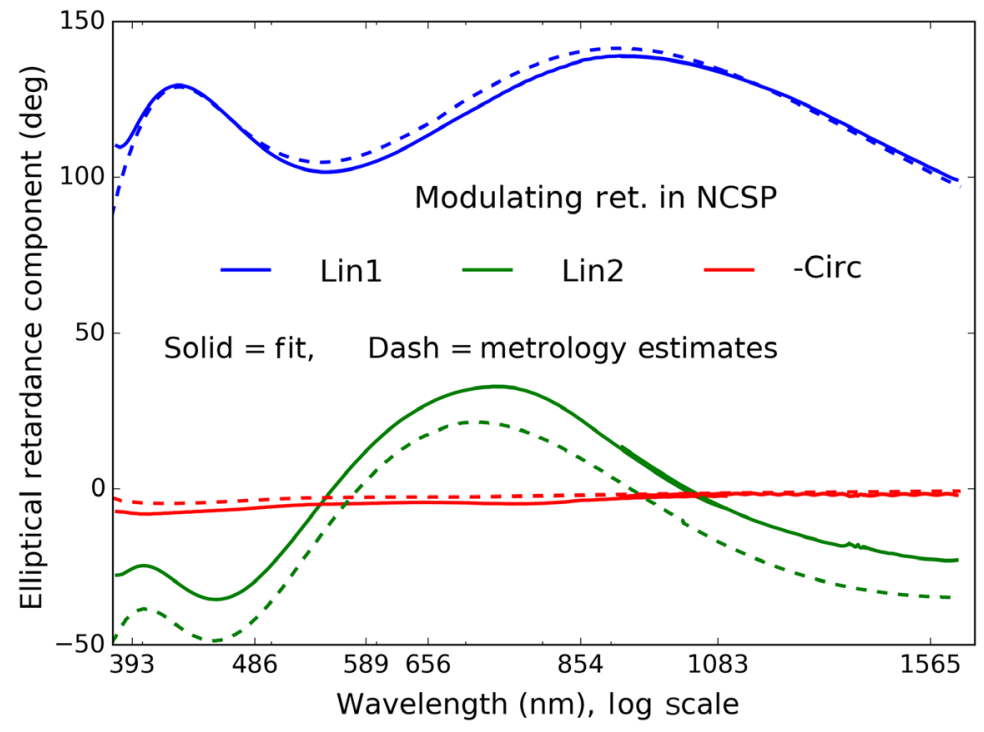

Fig. 14 The NCSP polycarbonate 5-layer modulator elliptical retarder metrology model (dashed lines) and 17-variable system fit results (solid lines). Note that the NLSP metrology had a sign flip on the circular component from $\mathrm{H} 20^{80}$

are shown in black. The metrology model is not particularly accurate as we did not attempt to account for some mirrors at significant fold angles, and we did not perform polarization metrology on other mirrors.

Figure 14 shows the three elliptical components fit for the NCSP modulating elliptical retarder. The dashed lines show the lab metrology (see $\mathrm{H} 20^{80}$ ). When the 2 mirror variables and the 3 modulator elliptical retardance variables are combined, the 5 variables can be used to create the 24 modulation matrix elements.

\subsection{Modulation Matrix Elements: 24 Variables}

Within the 36-variable system model, the 24 modulation matrix elements are entirely unconstrained free variables. In the 17-variable system model, the 24 modulation elements are derived from only 5 variables. We see very similar fit outputs for the modulation matrix elements in these two scenarios. The 24 modulation matrix element variables are within \pm 0.1 to better than \pm 0.05 of the more constrained 5 modulation variables as substantial functions of wavelength. 


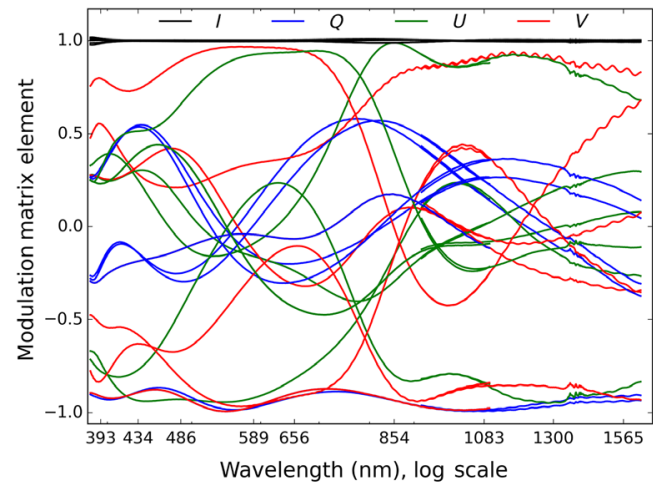

(a)

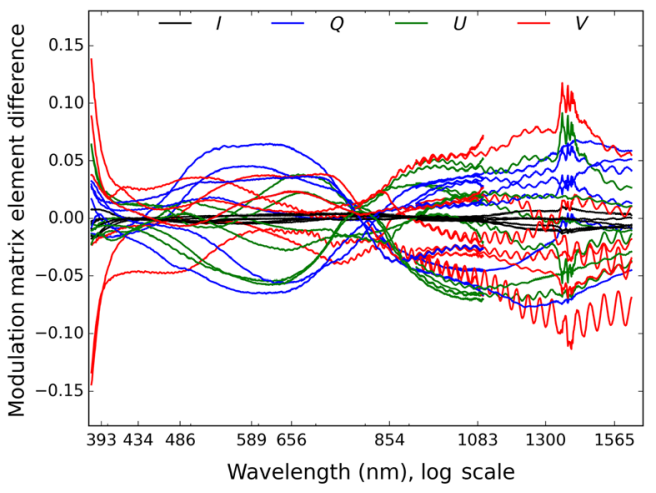

(b)

Fig. 15 (a) Modulation matrix elements fits for both VIS and NIR spectrographs using the 36variable system model. (b) The difference between the 36-variable and 17-variable system model modulation matrix elements. There are six blue curves for $Q$ modulation, six green curves for $U$, six red curves for $V$, and six black curves for $I$. The orthogonal analyzation difference between spectrographs shows good agreement in the wavelength region of overlap.

We show the 24 modulation matrix element variables Fig. 15(a). We color each element spectrum by the four Stokes parameters being modulated. Each color has six curves, one for each modulation state. The $I$ terms are not all at or below 1 as we did not enforce modulation matrix intensity normalization.

Fig. 15(b) shows the difference between modulation matrix elements for the 24 modulation variable versus 5 modulation variable models. We discuss this in detail later in Appendix D.3. The low magnitude of the element differences shows that we can recover all 24 matrix elements close to a very constrained physical model of just $Q$ diattenuation and $U V$ retardance for a grouping of 10 mirrors followed by a three variables for a rotating elliptical polycarbonate retarder. The similarity between these modulation matrices gives confidence in our physical model above in Figs. 13 and 14.

At infrared wavelengths, we can see some influence of the CalRet fitting errors coupling into the modulation matrix elements. We note the spectral oscillations in modulation as being caused by the clocking misalignment spectral oscillations from the CalRet (see $\mathrm{H} 20^{80}$ for details). We expect correlated errors both from the simultaneous global fit coupling these terms as well as the anticipated thermally forced spectral drift in these retardance oscillations driving photometric errors during the measurements. We also see some erratic spectral variation in the wavelengths impacted by atmospheric absorption near 1300 to $1450 \mathrm{~nm}$. This variable atmospheric transmission also causes photometric errors which couple into systematic errors of all model variables.

\subsection{Mirror Group Diattenuation and Retardance: Four Variables ( $\tau_{34}$ and $\left.\tau_{56}\right)$, $\left(\Delta_{34}\right.$ and $\left.\Delta_{56}\right)$}

We show the polarization properties fit for the two DKIST mirror groups articulated in elevation and azimuth in Fig. 16. Figure 16(a) shows the retardance of the two mirror groups in degrees phase. The thin dot-dashed lines show the initial guesses derived from the 17-variable system model fits above, barely distinguishable from the 36 variable fits. The dashed lines show a metrology model for the mirror groups derived using new Woollam ellipsometric measurements of the M3, M5, and M6 samples (see Appendix C for details on the ellipsometry). We have M4 measurements but the incidence angle is $\sim 4 \mathrm{deg}$ with negligible retardance. The dark green curve shows a single-mirror sample measured with NLSP from H19 ${ }^{67}$ at 45-deg incidence. As we showed in $\mathrm{H} 19,{ }^{67}$ there is substantial variability in the polarization each sample expected from typical manufacturing tolerances. The M3:M4 mirror group is essentially a single $45 \mathrm{deg}$ fold and we expect results similar to the ellipsometry and the NLSP metrology. For the M5:M6 model, we use ellipsometry measurements at the appropriate 15-deg and 30-deg incidence angles. There is some influence of $\sim 1 \mathrm{deg}$ retardance in the 1400-nm wavelength atmospheric absorption band for $\tau_{34}$. 


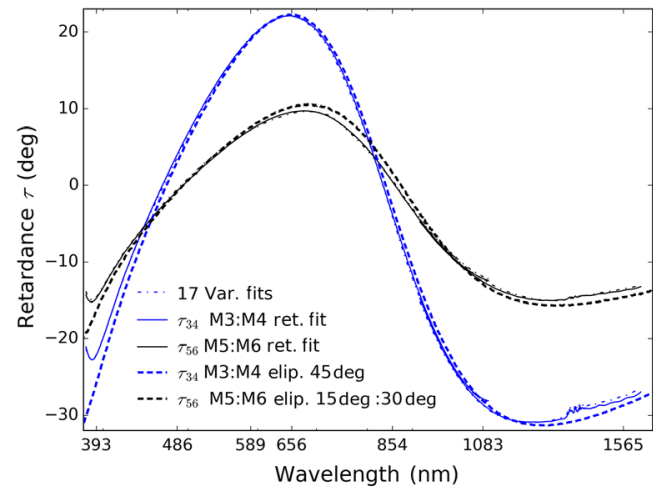

(a)

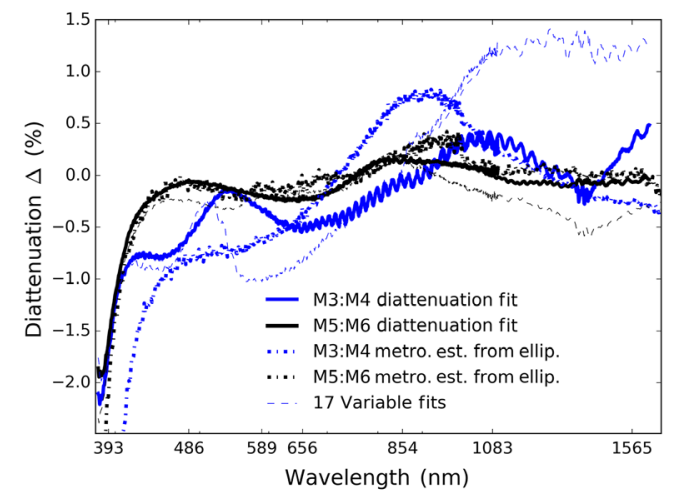

(b)

Fig. 16 (a) The DKIST mirror group retardance fit in degrees phase. Blue shows the M3:M4 group fit. Black shows the M5:M6 group fit. The solid lines show the 36-variable system model fits. The thin dot-dashed lines show the 17-variable system model fits barely distinguishable except at the longest wavelengths. The dashed lines show the metrology prediction for each mirror group based on the Woollam ellipsometry at the appropriate incidence angles. (b) The best fit to mirror diattenuation with the 36-variable system model as the thick solid lines. The thin dashed lines show the fits in the 17-variable system model scenario fits. The thicker dot-dashed lines show the diattenuation model derived from the ellipsometric data.

The mirror group diattenuation in Fig. 16(b) shows agreement well below $0.3 \%$ for the M5:M6 group between fitting scenarios. However, changes at 1\% levels for the M3:M4 group diattenuation terms are seen. We show the initial guesses used in the fit as the dashed lines computed as results from the 17-variable models fits above. We include ellipsometric measurements from Woollam as the dot-dashed lines (see Appendix C for details on the ellipsometry). The M3:M4 diattenuation is very significantly different above the noise levels with obvious coupling of retarder clocking oscillations into the best-fit $X$ values and diattenuation at $\sim 0.2 \%$ amplitudes. However, these mirrors are highly reflective and the general diattenuation signals are below $1 \%$ for all but the shortest wavelengths.

\subsection{Summary: System Model Fitting From 17 to 137 Variables}

These preliminary fits show that we can reproduce the 1872 NCSP measured fluxes to $0.5 \%$ levels with 62- and 137-variable models. The two software packages tested give comparable and stable results. The metrology for mirror coatings and retarders gives a very reasonable approximation of the system with the fit values close to expectations. We began with 17-variable model fits to show that the 24-variable modulation matrix matches metrology when fitting a grouping of mirrors and the rotating polycarbonate retarder installed in NCSP. The modulation matrix free parameters match the metrology model closely. This 17 -variable model had normalized photometric errors already at $1.3 \%$ for all 1872 exposures. The additional modulation degrees of freedom improved this fit to $0.8 \%$ normalized photometric error. We then introduced 26 free parameters representing fits for the unpolarized input flux as different from the estimate of the average of all modulated exposures without GOS polarizing optics in the beam (clears). These extra 26 variables improved the photometric errors by only $0.16 \%$ and did not perturb the prior 36 variables substantially. We show all the model variable changes between scenarios and all additional variable fits in Appendix B. The addition of 78 more retardance variables to account for thermal perturbation of the retarder increased the number of variables by more than $2.2 \times$ while only negligibly improving the photometric errors from $0.61 \%$ to $0.58 \%$, a relative change of only $5 \%$. The thermal behavior of the retarders and fitting for their time dependence requires more analysis and execution of already-planned thermal testing in upcoming observing runs.

The four DKIST mirror group parameters are the main output of this fitting phase. Both retardances and diattenuations are quite stable with the variable exchanges. These model outputs also agree well with multiple metrology tools. With this preliminary fit to the DKIST M3:M4 and 
M5:M6 groups, we can populate these terms of our system polarization database. Then we proceed to show how a daily instrument calibration would work using this database to derive a modulation matrix and several auxiliary parameters when doing a limited fit on a single PolCal. We plan substantial future work toward cross comparing instruments and identifying the limiting errors when DKIST begins observing with the suite of SPs presently undergoing installation.

\section{On-Sun Calibrations: Modulation Matrix and GOS Retardance}

The daily calibrations for the DKIST instrument modulation matrices are anticipated for multiple wavelengths and configurations. Calibrations use the best-fit mirror group model parameters for the telescope as outlined in the previous section. These daily fits always derive a modulation matrix along with fits to several calibration optic parameters. The same modulation matrix should be derived regardless of which CalRet or sequence is used within the error limits. Stability analysis after installation may suggest some instruments or configuration modes may be more stable and require less frequent calibration. Derivation of an instrument modulation matrix involves multi-variable fits to at least one PolCal data set. We outline some options for variable choices in Table 7. Fits now involve interaction with the DKIST system model database to look up the current value for the M3:M4 and M5:M6 mirror group polarization properties, shown as the first four rows. Depending on the stability of the DKIST primary and secondary mirror coating or the lamp mirrors, we can optionally fit or use database lookup values for the $Q U V$ partial polarization created by optics upstream of the GOS calibration unit for 0 to 3 variables. Similarly, we can fit variables or use database values for the transmission of the CalPol $\left(t_{\mathrm{pol}}\right)$ and CalRet $\left(t_{\mathrm{ret}}\right)$. Retarder properties, if stable, can also become database lookup tables. The nominal procedure tested here is to fit for the transmission of the two GOS optics, three elliptical parameters for the CalRet, and a full modulation matrix. The most DKIST instruments anticipate anywhere from 4 to 10 modulation states giving a range of modulation matrix elements from 16 to 40 variables.

The DKIST first light instruments have options for continuously rotating or discretely stepped modulation of the various retarders. In the case of VTF, the liquid crystal-based modulator is electrically controlled. The minimum number of modulation state variables is 16 to solve for the 4 Stokes input vector components with 4 photometric measurements. For NCSP, here we used 6 modulation states for 24 variables. Nominal defaults for ViSP, DL-NIRSP, and CN are between 6 and 10 states for 24 to 40 modulation matrix element variables. We obtained DKIST calibration data observing solar disk center on three separate days in August 2020. We compare those on-Sun data set calibrations with some derived using the GOS lamp.

Table 7 PolCal inputs.

\begin{tabular}{lc}
\hline \hline Name & Description \\
\hline$\tau_{34}$ & Database \\
$\Delta_{34}$ & Database \\
$\tau_{56}$ & Database \\
$\Delta_{56}$ & Database \\
$Q(U V)_{\text {in }}$ & 0 to 3 vars \\
$t_{\text {pol }}$ & 0 to 1 var \\
$t_{\text {ret }}$ & 0 to 1 var \\
$I_{\text {sys }}$ & 1 var \\
CalRet & 3 vars \\
Mod mat & 16 to 40 vars \\
\hline \hline
\end{tabular}


Different instruments sample the DKIST beam at different field angles. Instruments all have opto-mechanical methods to select a particular field offset from the telescope bore sight by using slit stepping or scanning mirrors mounted near pupil planes. We estimated the dependence on field angle for a range of DKIST configurations in HS17. ${ }^{61}$ For this preliminary commissioning model, we ignore the field of view dependence for the DKIST M3:M4 and M5:M6 mirror groups. We do, however, fit the field dependence of the modulation matrix with the option to fit all spatial and spectral pixels of all instruments separately. The DKIST polarimetry modules will calibrate whatever wavelength or field angle provided the variables and lookup table values. As we showed in HS17, ${ }^{61}$ the incidence angle range on M3 is 4.4 deg peak to valley across the beam center footprint. However, the incidence range for chief and marginal rays varies by only $0.38 \mathrm{deg}$ on M3 for the full 5.0-arc min field angle DKIST beam. This increases to $0.9 \mathrm{deg}$ for M5 and M6 following the off axis parabola M4. These range of angles create small cross talk variations of roughly \pm 0.02 in the system Mueller matrix elements at the extreme field edge (see for example HS17 $7^{61}$ Figs. 18 and 19).

\subsection{Daily PolCal Fitting Variables: Calibrator Elliptical Retardance}

Presently, the three elliptical CalRet parameters are fit simultaneous with the modulation matrix elements for every instrument calibration (called a PolCal). We assess fit quality here by taking the 26 lamp PolCal data sets used to derive the system model and use them to individually derive fits to the NCSP modulation matrices along with the associated CalRet and polarizer properties per Table 7. We show the 26 different elliptical retarder fit results for the retardance of the ViSP SAR derived with the 10-state sequence in Fig. 17(a). The NLSP metrology curves are also showed as the three dashed lines of the same color. The metrology is nearly indistinguishable from the fit results. We also note that we fit a 16-state PolCal data set collected at the same time and also find nearly identical results.

We also show the elliptical retardance derived for OCcal along with our NLSP metrology in the middle graphic of Fig. 17. This fit using the DKIST group model retardance values gives us results very near our NLSP lab metrology from $\mathrm{H} 20^{80}$ shown as the set of three dashed lines. We note that we get the same results to better than one part in 10,000 when running the fits with slightly perturbed different initial conditions. We have used the 17-variable, 62-variable, and 137-variable system models as initial guesses. In particular, the bottom curve in Fig. 17(b) compares 137-variable module fits with a lab metrology-based initial guess and using the 36-variable

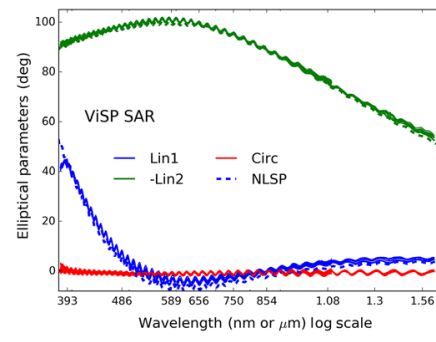

(a)

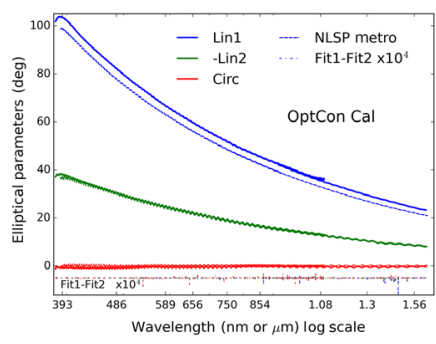

(b)

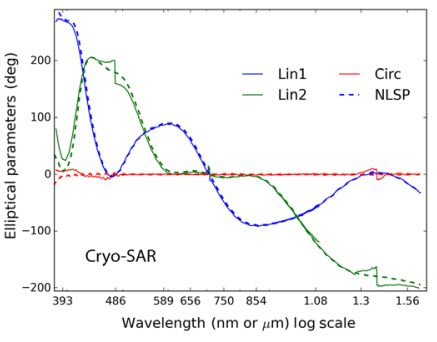

(c)

Fig. 17 (a) The elliptical retardance parameters fit to the 26 different PolCals for the 26 different data sets recorded with the ViSP SAR using the 10-state sequence. The first linear component is shown in blue, the second in green, and circular retardance is in red. The three elliptical parameters fit to the NLSP metrology are also shown as the dashed curves of the same color, almost indistinguishable from the individual fits. Note that we reversed the sign of the Lin2 component for clarity. (b) The elliptical retardance parameters fit for the OptConCal for a single PolCal data set. The solid lines show a fit with the initial guesses as the ViSP SAR, very far from the final value. The dashed lines show our NLSP metrology from the aperture center spatial map from $\mathrm{H} 20{ }^{80}$ The thin dot dashed line compares the fits to the system model with different initial guesses multiplied by 10,000 showing the fits converge to nearly identical retardance values to better than 0.001 deg retardance. (c) Parameters fit to the CN SAR with dashed lines showing NLSP metrology in $\mathrm{HS} 18 \mathrm{a}^{63}$ and spatial mapping in $\mathrm{H} 2 \mathrm{O}^{80}$ 
model outputs as initial guesses. The algorithm converges to an identical fit result to better than four decimal places for an error of less than a few parts per million.

We show the elliptical retardance spectra fit to 10-state calibrations with the 6-crystal $\mathrm{MgF}_{2}$ retarder called the CN SAR in Fig. 17(b). We choose a particular elliptical retarder model that forces the magnitude to go through zero near $700 \mathrm{~nm}$ wavelength. We note that this retarder for our 10-state CS goes through zero V calibration efficiency near 0.5 and $1.4 \mu \mathrm{m}$ wavelength (see $\mathrm{H} 20,{ }^{80} \mathrm{Fig}$. 16). These many variable model fits are poorly conditioned for some bandpass about these values. We also note that we do not expect a retarder model fit to the retarder Mueller matrix to be smooth and continuous (see Chipman Ch 28). ${ }^{88}$ We simply choose this model for convenience.

\subsection{On-Sun Calibrator Retardance Temporal Drifts}

The stability of the CalRet is a major limitation to overall accuracy. Fits to the calibration retardance were stable in time over roughly $30 \mathrm{~min}$ each to better than $0.5 \mathrm{deg}$. The OCcal in particular was better than 0.2 deg and mostly noise limited. We show the retardance temporal drifts in Fig. 18. Figure 18(a) shows the ViSP SAR retardance temporal drifts. Figure 18(b) shows the OCcal. There is little spectral variation of significance. The atmospheric absorption band around 1300 to $1450 \mathrm{~nm}$ wavelength clearly introduces \pm 2 deg errors. But the derived fits do not show time-dependent drifts of spectral clocking oscillations (though the spectral oscillation temperature sensitivity was shown in $\mathrm{H} 20^{80}$ ). This shows quite good spectral stability as well as verifying the predictions that the OCcal would be substantially more stable than the six-crystal ViSP SAR retarder.

\subsection{Daily PolCal Fitting Variables: Modulation Matrix Elements}

The 24 elements of the modulation matrix represent the many optics starting at M7 and ending at the detector element through the analyzer. The modules should remove all geometrical effects, deriving identical modulation matrix element spectra regardless of which CalRet, which CalPol, which CS, which mode (PolCal versus GroupCal), etc. We compare modulation matrices derived from three observations each using two retarders in Fig. 19. The data were collected on August 14, 2020, tracking solar disk center. Each calibration took roughly $10 \mathrm{~min}$. Azimuth and elevation angles changed by $<2$ deg during the data collections with the coudé table changing by about twice this amount when using the El-Az tracking mode. Three data sets were recorded

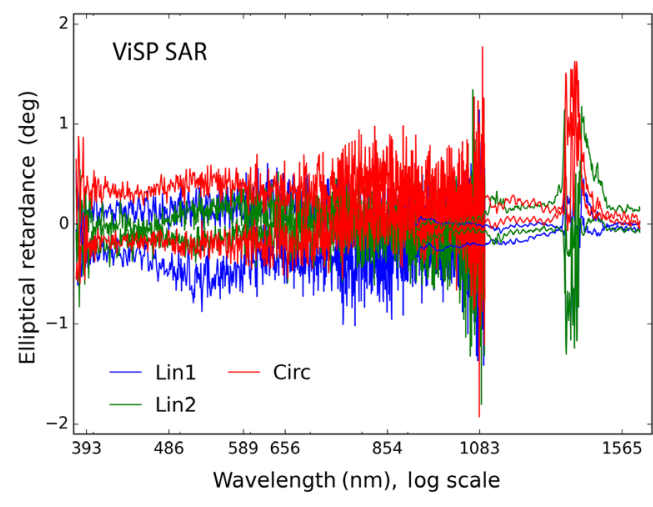

(a)

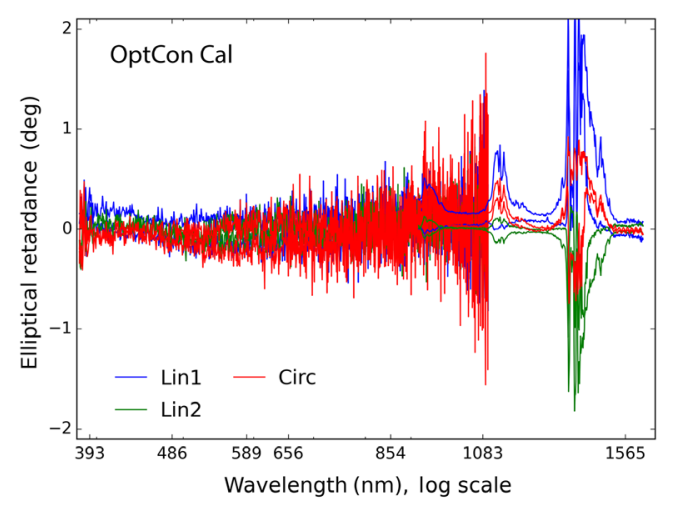

(b)

Fig. 18 The elliptical retardance parameters fit change in time computed as the difference between the first fit and subsequent fits. (a) The ViSP SAR elliptical retardance parameter drifts and (b) the OCcal elliptical retarder parameter drifts. Blue shows the first linear component, green shows the second linear component, and red shows the circular component. The atmospheric transmission band variability around 1300 to $1450 \mathrm{~nm}$ wavelength drives errors up to \pm 2 deg. The ViSP SAR in (a) shows some statistically significant differences above shot noise levels at \pm 0.5 deg magnitudes. The OCcal shows smaller differences at 0.2 -deg magnitudes, mildly above shot noise limits. 


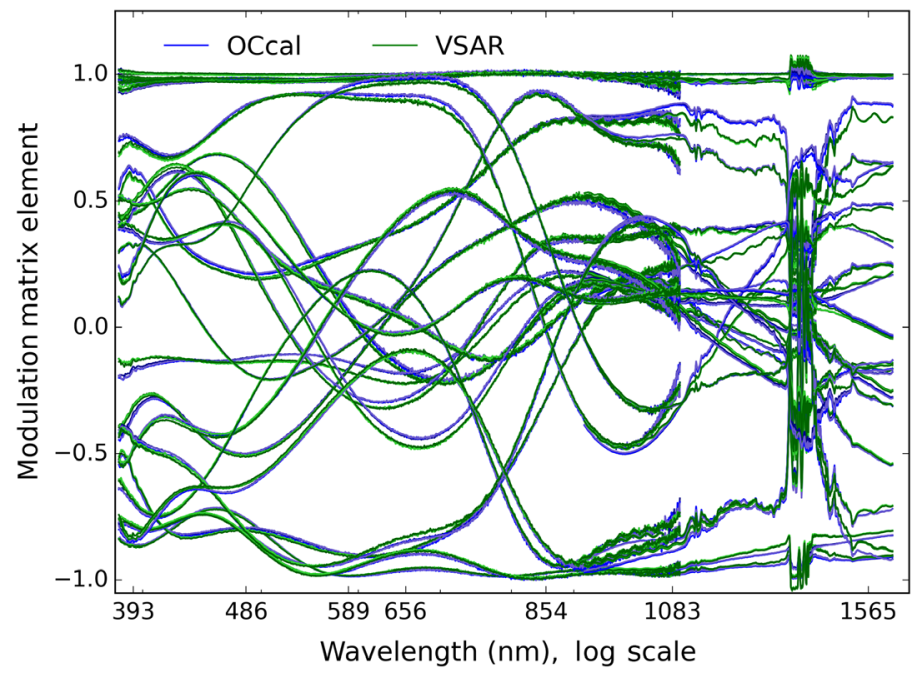

Fig. 19 The three on-Sun derived modulation matrices for two retarders (six curves total). The three ViSP SAR derived matrices are shown in green shades. The three OCcal derived matrices are shown in blue shades.

contiguously with the ViSP SAR and then three more data sets were recorded with the OCcal. Each set was fit independently. The blue curves show the 24 modulation matrix elements derived with the three OCcal data sets. The green curves show the elements derived with the three ViSP SAR data sets. The instability in the 1300- to 1450 -nm wavelength atmospheric absorption band causes much more severe fitting issues than other atmospheric features. On this particular day, there were clouds at observatory level within a few hundred yards of the facility. We anticipate improvement in stability on more dry days with a stronger atmospheric inversion layer suppressing convection around the observatory. Additional small mismatch between fits can be seen where the VIS and NIR spectrographs overlap.

\subsection{On-Sun Modulation Matrix Temporal Drifts}

The temporal drifts in the three repeated fits to the modulation matrix elements are shown in Fig. 20(a). Differences were computed by subtracting the first fit from the subsequent fits

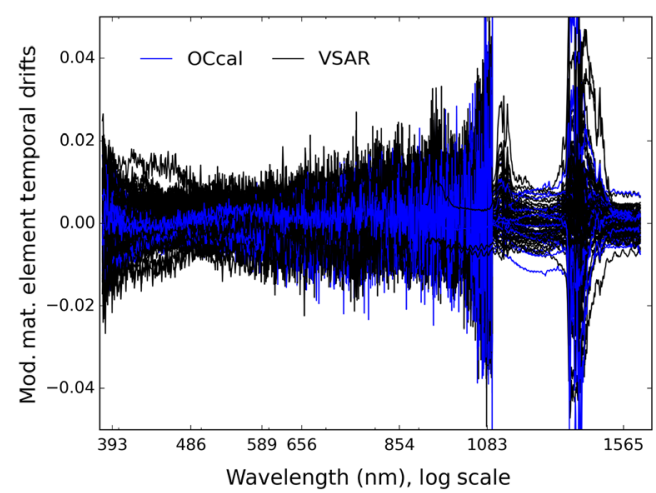

(a)

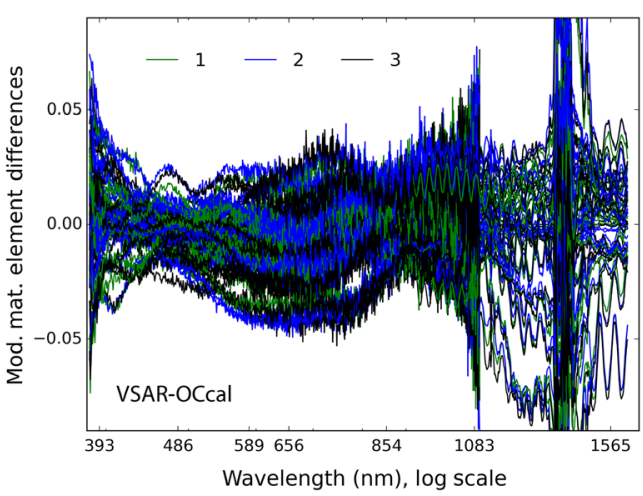

(b)

Fig. 20 (a) The on-Sun derived temporal drifts in three subsequent modulation matrix fits for both the VSAR shown in black and OCcal shown in blue. (b) The difference between on-Sun derived matrix elements comparing the two retarders computed as VSAR minus OCcal for three subsequent repeated measurements. The fits should separate modulation from calibrator retardance completely with no systematic error above shot-noise limits. Atmospheric absorption influences the large band of errors near $1300 \mathrm{~nm}$ wavelength. 
representing changes of roughly 10 and 20 min separately for each CalRet. In Fig. 20(b), we compare the temporal differences between modulation matrix elements derived with the two different retarders. Differences were computed as the fit using the ViSP SAR subtracted from the fit using the OCcal. All three fit pairs were separated by roughly half an hour.

\section{Summary}

This paper presents successful preliminary full-system PolCals of DKIST. A metrology tool called the NCSP was designed, built, and deployed to measure DKIST system PolCals over the 390-to $1600-\mathrm{nm}$ bandpass while also providing a highly accurate absolute polarization reference frame. Data both on-Sun and with the DKIST calibration lamp were collected and calibrated. Fits of up to 137 variables compare very well with metrology.

We showed design and fabrication elements of the NCSP in Sec. 2. A new custom master polarizer with $\pm 0.065 \mathrm{deg}$ orientation accuracy when combined with laser tracker equipment and on-telescope installation procedures was built and used on-Sun. This gives the DKIST polarization reference frame a high orientation accuracy. Preliminary fits were presented in Sec. 3 showing absolute orientation alignment of the two current DKIST alignment polarizers using both the DKIST VBI as well as NCSP. We note that the spatial variation of the angle of polarization is below \pm 0.03 deg with a gradient-like spatial pattern along with a wavelength dependence of similar magnitude. This is far smaller than the alignment tolerances for our master polarizer presented in Sec. 2. The transmission spatial variation couples into photometric errors for beam footprints away from the optical bore-sight, as discussed in Sec. 4.

The NCSP recorded DKIST system calibrations using the Gregorian focus ALS following procedures similar to what many astronomical telescopes would perform. We derived mirror group polarization parameters. The fitting outputs showing successful comparisons with metrology covering relatively simple 17-variable fits to complex 137-variable system models. Section 4 outlined the basic method for fitting and data processing. Section 5 showed system models of increasing complexity and the associated improvement in photometric fitting errors. Section 5 also compared DKIST mirror group retardance and diattenuation with new ellipsometric metrology results as well as our in-house system metrology presented in $\mathrm{HS} 17,{ }^{61} \mathrm{H} 19,{ }^{67}$ and, $\mathrm{H} 20{ }^{80}$ The transmission and retardance fits to the suite of DKIST CalRet s compared well with metrology presented in Secs. 4 and 5, as well as our prior metrology results in HS18a, ${ }^{63}$ $\mathrm{HS} 18 \mathrm{~b},{ }^{66}$ and $\mathrm{H} 20{ }^{80}$ We also created an updated NCSP and DKIST mirror group model shown in Appendix C. We used this to compare the NCSP instrument modulation matrix with constrained and unconstrained models. Some mild photometric error can be caused by the modulator beam deflection. Our metrology efforts have provided estimates for all 137 model variables with all fits close to expectations and metrology uncertainties. We also outlined a perturbation analysis to demonstrate photometric error changes and fit outputs in response to orientation changes for the DKIST CalPols.

The normalized photometric fitting errors shown in Sec. 5 improved from 1.3\% when using only 17 variables to better than $0.6 \%$ when using 137 variables. A 62-variable model that did not fit for CalRet time dependence (through thermal forcing) did almost as good with $0.61 \%$ normalized photometric fitting errors. We showed in Sec. 5 that the photometric errors are reasonably approximated by a Gaussian distribution with no major outliers. The fits are stable and all data sets contributing to the fit have roughly equal photometric error following a simple statistical distribution.

We presented the first solar PolCal observations with DKIST in Sec. 6. We followed the anticipated DKIST calibration procedure where we derive an instrument modulation matrix as well as fits to the calibration optic parameters using a single CS. The fit outputs are stable in modulation matrix element to better than \pm 0.01 . The fit elliptical retardance parameters are also stable to \pm 0.2 deg for the OCcal with \pm 0.5 deg stability derived for the ViSP SAR. We outlined in Sec. 4.2 several significant optical limitations to these preliminary results. As more DKIST observations are collected, we anticipate a more thorough analysis of longer term stability and limitations of fitting accuracy when including or not many variables outlined here in Sec. 5. 
We extend our conclusions about retarder thermal properties by showing direct-attach thermal measurements as well as multiple IR remote temperature measurements on a quartz test retarder (QTR) as well as all DKIST CalRets in Appendix B. We showed the three $\mathrm{SiO}_{2}$ retarders both heat roughly $6^{\circ} \mathrm{C}$ in $30 \mathrm{~min}$ of exposure to the $300-\mathrm{W}$ DKIST beam with more of that temperature rise concentrated as $4^{\circ} \mathrm{C}$ in $10 \mathrm{~min}$. All three $\mathrm{SiO}_{2}$ retarders saw roughly $6^{\circ} \mathrm{C}$ change in 12 min of cool down. The $\mathrm{MgF}_{2}$ retarder, as predicted in $\mathrm{HS} 18 \mathrm{a},{ }^{63}$ showed much lower heating response of $1.5^{\circ} \mathrm{C}$ in $30 \mathrm{~min}$. These retarder temperature drifts drive several possible error sources. We showed in $\mathrm{H} 20^{80}$ that the largest term was the spectral drift of retardance spectral oscillations caused by the retarder pair clocking misalignments. We also showed in $\mathrm{HS} 18 \mathrm{a}^{63}$ and

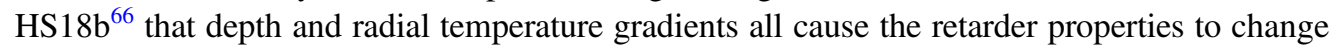
with time. We showed in $\mathrm{HS} 18 \mathrm{~b}^{66}$ spatial variation of retardance produces errors when using a rotating retarder away from the optical bore-sight. However, our on-Sun calibrations of Sec. 6 showed that we achieved \pm 0.2 deg fitting stability when using a beam \pm 30 arc sec field angle away from DKIST bore-sight over $30 \mathrm{~min}$ of solar illumination.

The system model presented here for DKIST shows how a range of metrology tools can be combined with $>100$ variable system model fits to provide a well constrained physical model of a very complex optical system. We showed the photometric improvement and perturbation analysis for various options for fitting a range of physical properties. These variables all have a physical basis such as thermal dependence of retarder properties or uncertainties in reference polarizer orientation. This preliminary system model has several areas for improvement and continued analysis outlined in this paper anticipated in the near future as the observatory and several facility spectropolarimetric instruments come online.

\section{Appendix A. Model Fitting Output Stability and Perturbations}

We highlight in this section additional model fitting outputs and comparisons with metrology. In particular, we show the additional various transmission functions derived for the GOS polarizers and retarders at $F / 13$ compared with the collimated lab measurements sometimes showing fringes.

\subsection{A.1 System Model Fits-QUV Input Beam Polarization: Three Variables}

The GOS lamp consists of a quartz tungsten halogen bulb fitted inside an $F / 3$ elliptical reflector with a protected silver coating. A theoretical three lens afocal zoom system was conceptually designed to relay the $F / 3$ beam to the $F / 13$ Gregorian focus. The last lens was substituted with two commercial condenser lenses for a total of four lenses in the design. To package this system in the very confined space of the GOS enclosure, a 45-deg incidence fold angle mirror was placed after the second lens in a diverging beam. An Edmund Optics protected silver-coated mirror was used for this fold, having polarization properties as shown in Appendix C.6. The mirror diattenuation did rise strongly toward short wavelengths but the elliptical reflector and four other lenses complicate predicting the polarization of this lamp input beam. Figure 21 shows the GOS lamp input beam fit $Q U V$ spectra as blue, green, and red lines, respectively. The 17variable fit is included as the dashed lines and was also used as the initial guesses for the 36-variable fits. The 36-variable model fit output is shown as the solid lines. The 62-variable model fit is shown as the thicker set of dashed lines. There is up to $1 \%$ difference between the $Q$ fits for the 17-variable case and subsequent cases. The $U$ and $V$ fits are in general smaller in magnitude and more stable with an increasing number of model variables. We note though that the 36, 62, and even subsequent 137-variable models (not shown) do not differ by more than a small fraction of a percent for this partially polarized elliptical input beam.

\subsection{A.2 System Model Fits-GOS Transmission: Two Variables}

Figure 22 shows the transmission derived for the CalPol in black and for the CalRet (ViSP SAR) in blue. The thin dashed lines show the 17-variable fit outputs. The thicker solid lines show the 36-variable fits. We do not show the 62-variable model fits or the 137-variable model fits as they

J. Astron. Telesc. Instrum. Syst. $\quad$ 018004-32 Jan-Mar 2021 • Vol. 7(1) 


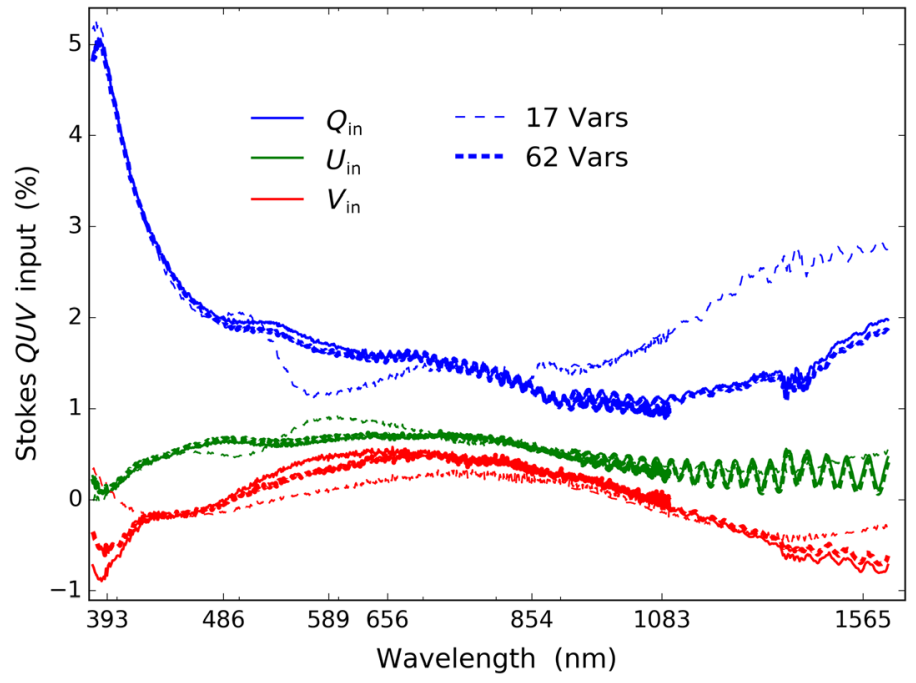

Fig. 21 The partial polarization parameters for the input Stokes vector created by the lamp optics. Blue shows $Q_{\text {in }}$, green shows $U_{\text {in }}$, and red shows $V_{\text {in }}$. The solid lines show the nominal 137variable system model scenario. Dashed lines show the 62-variable scenario, nearly indistinguishable from the solid lines. Dot-dashed lines show the 17-variable secnario.

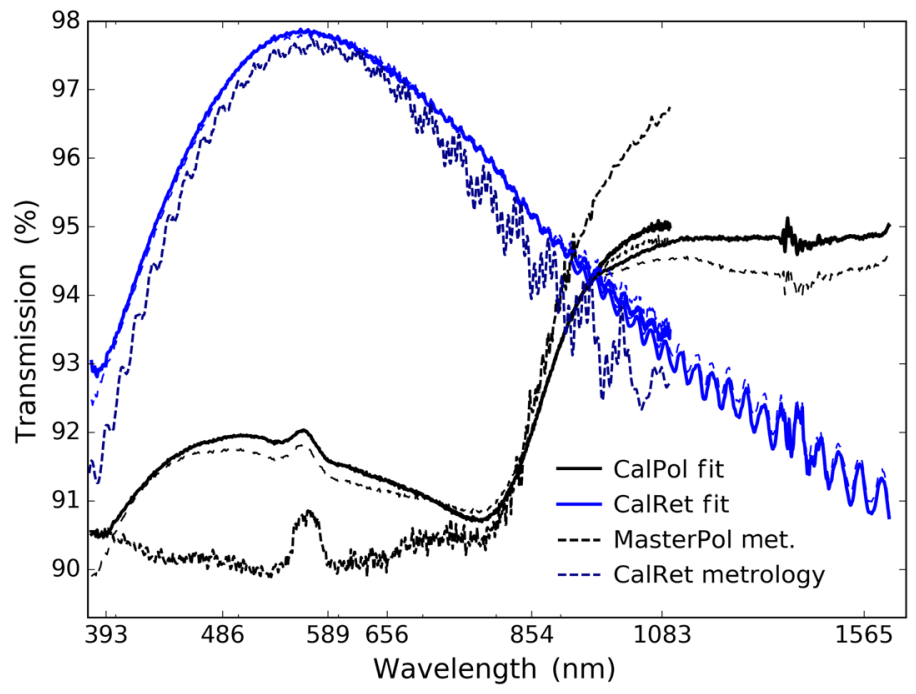

Fig. 22 The global transmission functions fit to all 26 PolCal sequences during the mirror group model. The CalPol transmitted linear polarization state is shown as black. Fits to 17-variable models are shown as thinner dashed lines. Fits to the 36-variable model are shown as solid lines. The ViSP SAR retarder is shown as blue. Lab metrology comparisons are shown as dot-dashed black and dark blue.

are only different at magnitudes below $0.02 \%$ transmission. We also show as dot-dashed dark blue lines the NLSP metrology results from the laboratory spatial Mueller matrix mapping using a collimated input at few millimeter diameter. This collimated metrology is more sensitive to the interference fringes compared to the $F / 13$ DKIST Gregorian focus beam per H18, HS18a, and $\mathrm{H} 20 .^{63,63,80}$ This is separate from the clocking misalignment spectral oscillations in the CalRet that give rise to correlated errors in transmission seen in the system model fits particularly at longer wavelengths per $\mathrm{H} 20 .{ }^{80} \mathrm{We}$ do not have lab metrology on this particular CalPol, so we graph transmission of one linear polarization state through the master polarizer as the dot-dashed black lines. These two polarizers are of the same type (UVT-240a) and with the same conformal protective coating. Some small differences are expected. 


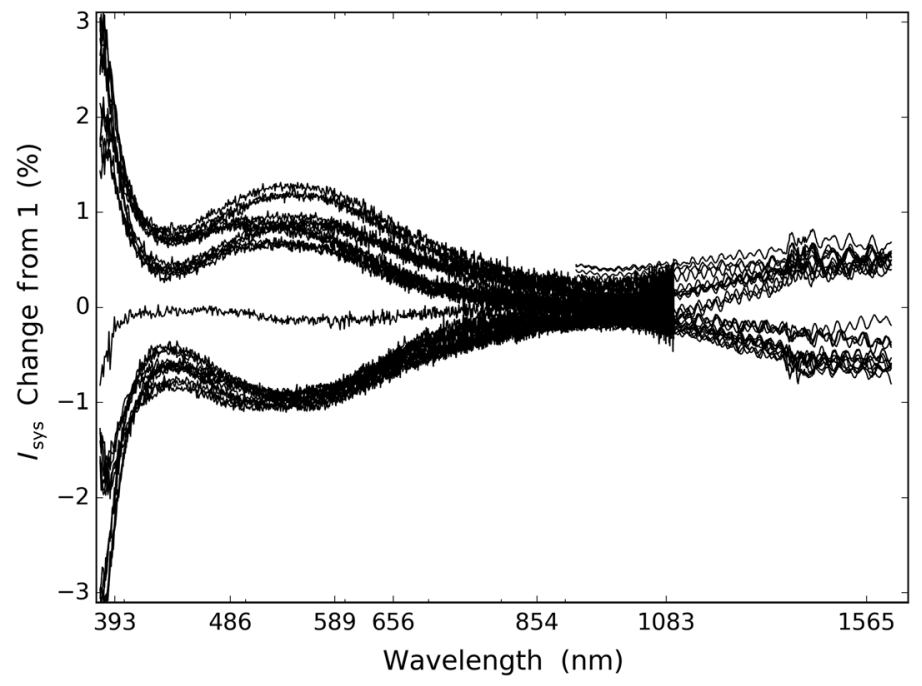

Fig. 23 The change in $I_{\text {sys }}$ in percent from the average of the modulated clear flux for the 62-variable model.

\subsection{A.3 System Model Fits-I $I_{\text {sys }}$ : 26 New Variables for a 62 Total Variable Case}

We add 26 new variables to the 36-variable model scenario by including fits to the unpolarized system intensity value $\left(I_{\text {sys }}\right)$ for each PolCal block of 72 spectra. This increases the total number of variables from 36 to 62 . As we normalized our data set by the mean of the modulated clear exposures, when we add this degree of freedom, we are fitting the change from 1 caused by the difference between the unpolarized system flux $\left(I_{\text {sys }}\right)$ and the mean of the clears. Figure 23 shows the change to the $I_{\text {sys }}$ values fit in the 62 -variable model. These $I_{\text {sys }}$ values show the difference between the average of the modulated clear exposures was roughly $1 \%$ from the best fit value for the unpolarized flux component for the Stokes vector entering the system. Each PolCal is recorded over a range of telescope geometries with significant symmetries. These differences nominally scale with the partial polarization of the lamp input spectrum. There is substantial symmetry in Fig. 23 as we only observed at elevations of 15 deg and 75 deg while using a pattern with integer multiples of 90 deg in Azimuth and coudé table angle.

\subsection{A.4 137 Variable Models: 78 Separate Calibration Retarder Elliptical Parameters}

We anticipate thermally forced drifts in retardance parameters for the CalRet as well as coupling of photometric errors from many variables into the retardance parameters. The calibration lamp is a major thermal source in the GOS enclosure. Our three elliptical parameter fits to the ViSP SAR during the 17, 36, and 62 model scenarios fit our previously reported lab metrology rotated by 23 deg combined with -1 multiplying the first linear component ( $\mathrm{HS}_{18 b^{66}}$ and $\mathrm{H}_{20} \mathrm{O}^{80}$ ). We note differences between model fit outputs are $<3$ deg magnitude with very clear fitting of the clocking error induced spectral oscillations.

We have shown several examples of thermal impacts on the retarder in HS18a ${ }^{63}$ and HS18b. ${ }^{66}$ We detail in Appendix B below the significant thermal testing done with the DKIST CalRets and polarizers in January 2020. We show the difference between the 26 elliptical retarder parameters for each PolCal time and the single global fit to one elliptical retarder parameter set in Fig. 24(a) with the CalPol at the 87.2-deg orientation. Figure 24(b) shows the temporal change in each of the 26 individual retarder parameter set fits from the mean value of each elliptical parameter. We showed thermal simulations in $\mathrm{HS}_{18 \mathrm{a}^{63}}$ Appendix B as well as $\mathrm{H} 20^{80}$ Appendix C.7.3. We showed temperature gradients can induce spectral changes of a few degrees retardance in each component. We show some temperature measurements of our retarders under the $300-\mathrm{W}$ beam load as well as the lamp heating in Appendix B. We can also clearly see the atmospheric 


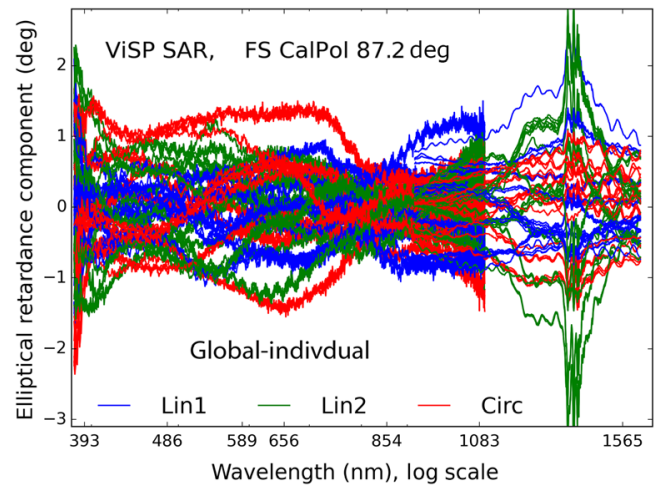

(a)

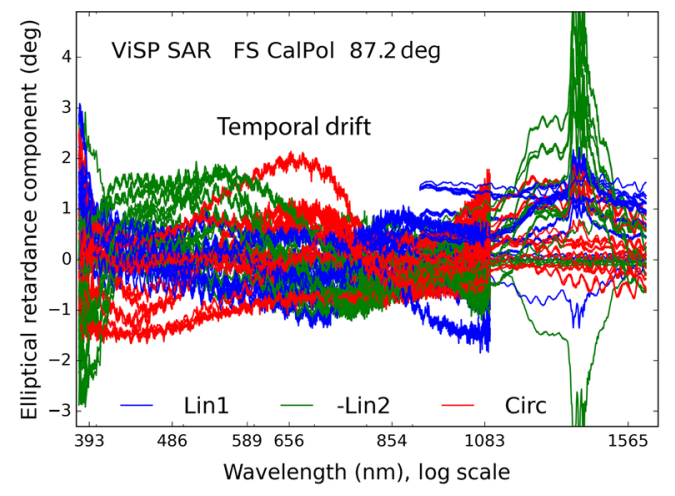

(b)

Fig. 24 (a) The difference between the single global retarder 3 variables and the 26 individual elliptical retarder fits with 78 total variables to the ViSP SAR. (b) The temporal drift of the three elliptical retardance parameters from the first PolCal. Both of these graphics show the model with the CalPol at $87.2 \mathrm{deg}$. The atmospheric absorption band near 1300 to $1450 \mathrm{~nm}$ have very large error levels. We do not find any significant temporal trends that correlate with our temperature measurements.

absorption bands more than double the retardance change in some cases showing the coupling between photometric errors and retardance changes.

\subsection{A.5 PolCal Fit: Transmission of GOS Polarizers and Retarders}

We show in Fig. 25 the fit results for the transmission of the CalRet and FS CalPol when using the ViSP SAR in Fig. 25(a). The 26 individual ViSP SAR transmission spectra should be the same as the globally fit single transmission spectrum derived from GroupCal. We also show the transmission from the single PolCal with the 16-state sequence using the ViSP SAR for comparison. Note for these tests the same polarizer (CalPoll Spare) was used for all tests.

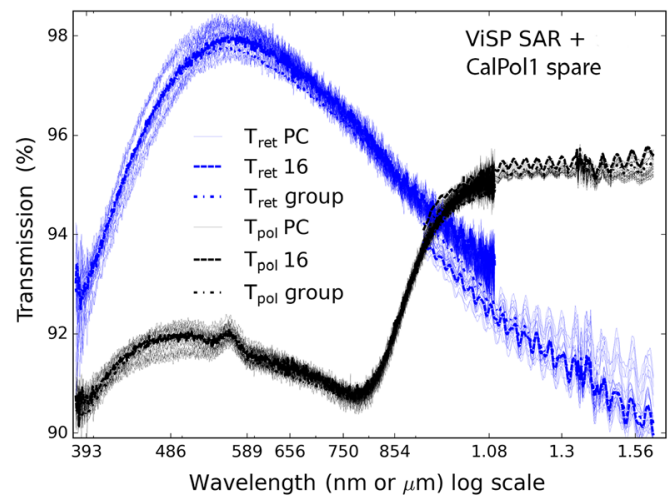

(a)

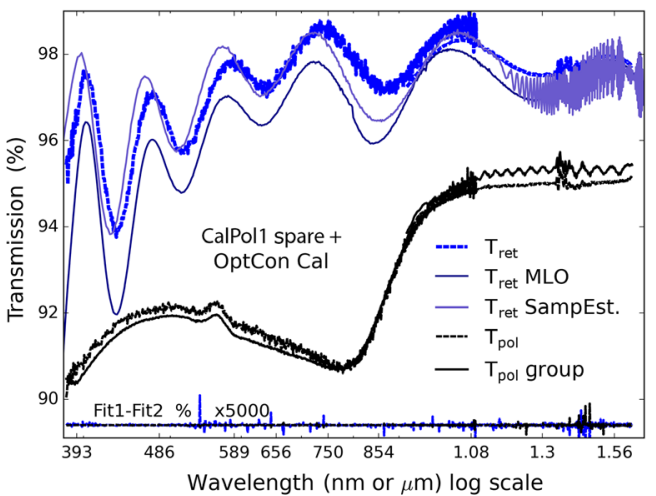

(b)

Fig. 25 (a) The transmission fit for the 26 different PolCals recorded with the ViSP SAR and the 10 -state sequence as thin colored lines. The thicker dashed lines show the individual PolCal fit to the 16-state sequence using the ViSP SAR. The dot-dashed line shows the globally fit transmission when doing the group calibration procedure to all 26 PolCals with the ViSP SAR and 10-state sequences. (b) The OptConCal transmission as dashed blue and CalPol1 Spare transmission as dashed black. Both curves are derived from the single 10-state PolCal. Dark blue shows the transmission measurement done by Meadowlark Optics (MLO) for the part after they performed the optical contact bond. The slate blue line shows the transmission estimated from thin BK7 coating witness samples. The globally fitted CalPol1 transmission derived from the simultaneous GroupCal fit to all 26 ViSP SAR data sets is shown for reference as $T_{\text {pol }}$ group. The dot dashed line compares the fits to the OptConCal data sets with different initial guesses multiplied by 5000. 


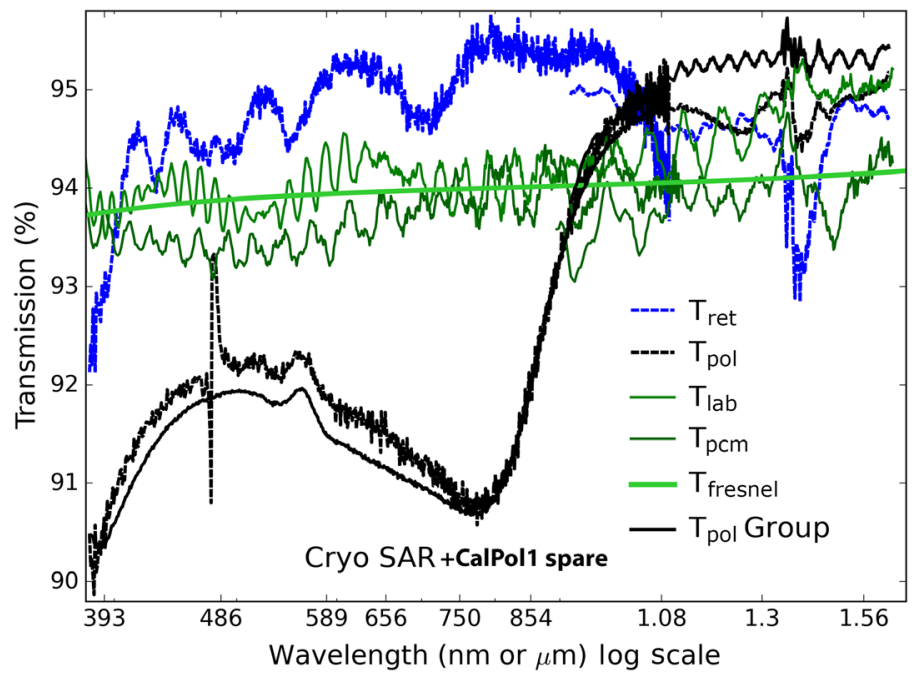

Fig. 26 The transmission fitted for the CryoSAR in blue. Lab metrology references in green. Theoretical transmission model in light green. CalPol1 Spare fit transmission in black. The CalPol1 Spare transmission derived during group model fit is the dashed black line. See text for details.

The OpConCal has its own transmission spectrum but the CalPoll spectrum derived should match that derived with any other retarder.

The optical contacted calibrator (OptConCal) is shown in Fig. 25(b) along with lab metrology. The PolCal fit transmission compares quite favorably with the metrology, matching to better than $1 \%$ absolute error and showing the same spectral oscillations expected from the coating witness samples. The dark blue line shows a Varian Cary transmission measurement for the optic done at Meadowlark Optics facility after they performed the optical contact bond on the coated quartz crystals. The slate blue line shows an estimate of the transmission when using 0.2-mmthick BK7 substrates as coating witness samples. The Varian Cary resolves some of the spectral fringes at wavelengths longer than $1 \mu \mathrm{m}$ for these thin samples. The dot dashed line shows the difference between the fits to the OptConCal data set when using different initial guesses. The fits converge to the same transmission values to roughly four decimal places. The Fit1-Fit2 curve is multiplied by 5000 to show erratic single spectral pixel errors at magnitudes of a few parts per 10,000. These very small residual differences show the fits converge to nearly identical transmission values to better than $0.0002 \%$ regardless of initial conditions. The globally fitted CalPol1 Spare transmission derived from the simultaneous GroupCal fit to all 26 ViSP SAR data sets is shown for reference as $T_{\text {pol }}$ group. Fits to this variable should agree regardless of retarder when fitting the same optic (CalPoll Spare).

We show the transmission derived for the CN SAR in the $F / 13$ DKIST beam in Fig. 26 as the thick blue line. The set of green lines show the lab transmission measurements for a collimated beam with this retarder mounted in our NLSP system along with the CN polychromatic modulator (PCM). The SAR and PCM should have identical unpolarized light transmission as both assemblies consist of six uncoated $\mathrm{MgF}_{2}$ retarders with the same five oil layers between the six crystals (see HS18a ${ }^{63}$ and $\mathrm{H} 20^{80}$ for details). The theoretical Fresnel losses accounting for all 12 optical interfaces in the crystal stack are shown in Fig. 26 as the bright green smooth line for $T_{\text {fresnel }}$. We also show the FSpol transmission derived from the same data set as the thicker black line. The thin black line is the same FS CalPol transmission derived during the group calibration fitting procedure to all $26 \mathrm{ViSP}$ SAR data sets is shown for reference as $T_{\text {pol }}$ group.

\subsection{A.6 Fitting Stability with Polarizer Orientation Error}

The fitted variables in the system model are highly coupled. One major uncertainty in the polarization error budget is the absolute orientation of the polarization coordinate reference, set by the 
master polarizer described in the main text. We show how the 36-variable system model fit variables change upon perturbation of the CalPol orientation by \pm 0.5 deg in Fig. 27. These fitted variables can change substantially with complex wavelength dependence, while little change in the photometric fitting error is seen. As we outlined in Sec. 5, the relative photometric error only changed by $5 \%$ from $1-\sigma$ photometric errors of $0.60 \%$ to $0.57 \%$. We show these curves to demonstrate the system model variable wavelength dependence and widely different sensitivities to the CalPol orientation. Though all variables are coupled, they reproduce the measured photometry similarly well.

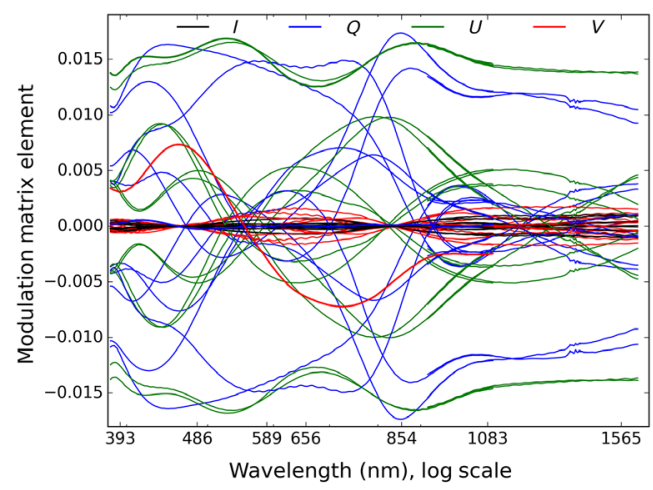

(a)

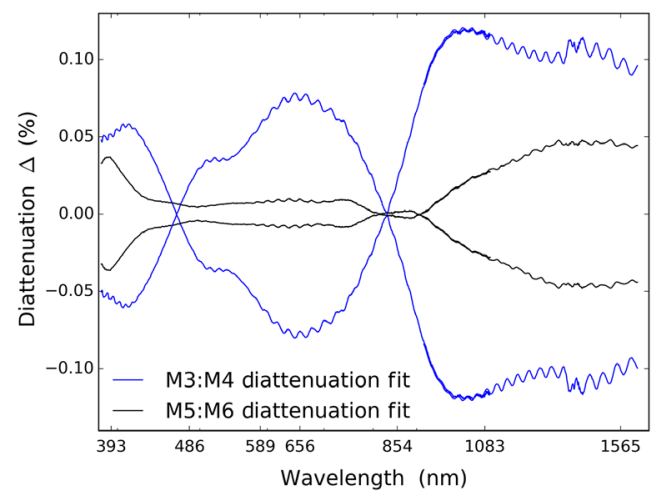

(c)

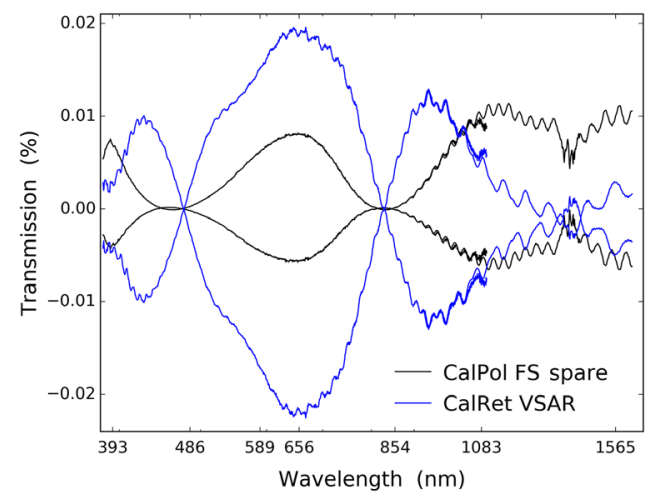

(e)

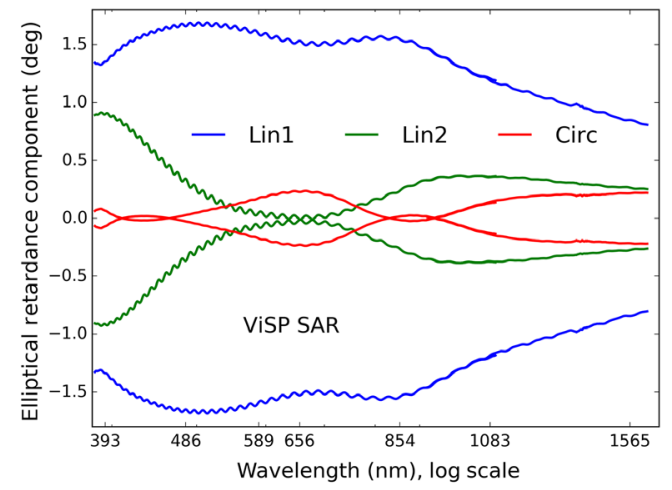

(b)

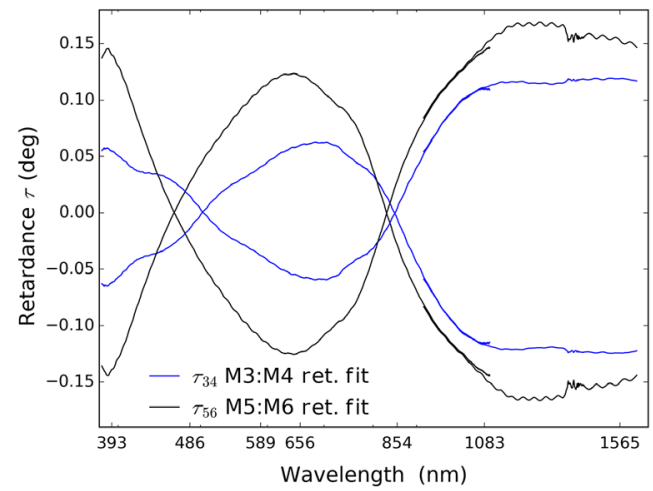

(d)

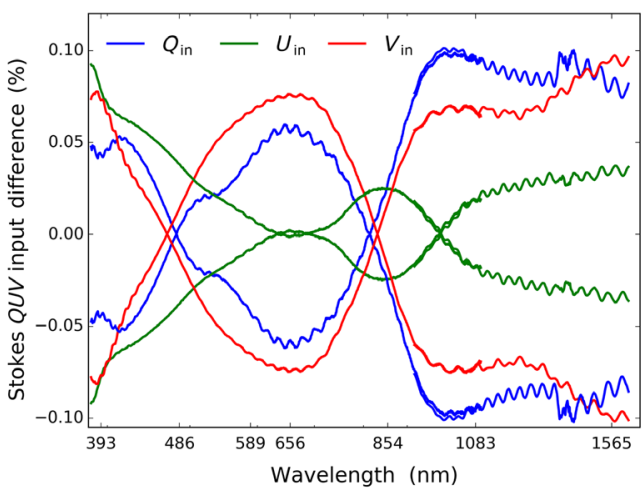

(f)

Fig. 27 The perturbation of the fitted variables in the 36-variable system model when changing the CalPol input orientation by \pm 0.5 deg. (a) The 24 modulation matrix elements; (b) the three CalRet parameters; (c) the DKIST mirror group diattenuation below $\pm 0.12 \%$; (d) the mirror group retardance changes below \pm 0.17 deg; (e) transmission of the CalPol and CalRet (ViSP SAR) at levels below $\pm 0.02 \%$; and (f) the lamp input $Q U V$ spectra below $\pm 0.1 \%$. We note the photometric errors did not substantially improve under this polarizer orientation perturbation. 


\section{Appendix B. Thermal Polarization Optic Testing}

Thermal remote sensing tools now provide direct feedback on optic temperatures relevant to astronomical instrument systems. Given the CalRet thermal concerns and many variables in finite element models, we installed sensors to monitor optic temperatures in real time along with historical data logging. We correlate the remote sensing tools with direct attach thermocouples (TCs) to show an example of retarder temperature monitoring to assess the optic and also provide assurance for data quality. DKIST was used during January 2020 to test the thermal performance of the polarization optics. We tested the QTR, described in $\mathrm{H} 20^{80}$ along with all DKIST CalRets and polarizers. We had installed temporary TCs directly onto the QTR surface as well as to the uncoated substrate side of the polarizers. We also used the IR sensors in the GOS. With these direct measurements as well as remotely sensed temperatures, we can assess the validity of our temperature models and set expected timescales for thermal drifts of retardance.

The GOS has two IR sensors measuring in the 8 - to $14-\mu \mathrm{m}$ wavelength range. The sensors are slightly different models with different optical beam diameters and footprints. The IR1 has a 20-mm spot leaving the lens with the spot size decreasing to $9 \mathrm{~mm}$ at $450 \mathrm{~mm}$ then diverging out again to $32-\mathrm{mm}$ diameter at $800 \mathrm{~mm}$ distance. This IR 1 sensor has a stated accuracy of $\pm 1 \%$ or $\pm 1^{\circ} \mathrm{C}$ The repeatability is a factor of two lower at $\pm 0.5 \%$ or $\pm 0.5^{\circ} \mathrm{C}$. The IR 2 sensor used a 22 to 1 focus ratio beam. The beam is only $7-\mathrm{mm}$ diameter leaving the lens with a footprint diverging to $9 \mathrm{~mm}$ at $200-\mathrm{mm}$ distance, $18 \mathrm{~mm}$ at $400 \mathrm{~mm}$, and $36 \mathrm{~mm}$ at $800 \mathrm{~mm}$. The $\mathrm{C} 1$ model has the same accuracy and repeatability in temperature with a $150-\mathrm{ms}$ response time. The sensor range is $-20^{\circ}$ to $180^{\circ} \mathrm{C}$.

The IR1 sensor beam hits the polarizer level $14.2 \mathrm{~mm}$ off of center with a 14-mm-diameter beam footprint. The IR 1 sensor beam hits the retarder level $21.5 \mathrm{~mm}$ of optic center with a 12 mm-diameter beam footprint. The IR1 sensor also likely can see the aperture edge for the master polarizer level with a 17-mm-diameter beam footprint decentered on the aperture by $49.8 \mathrm{~mm}$. The IR2 sensor beam hits the polarizer level decentered by $43.3 \mathrm{~mm}$ with a 15 -mm-diameter beam footprint. The IR2 sensor beam hits the retarder level decentered by $30.0 \mathrm{~mm}$ with an 18 mm-diameter beam footprint. The IR2 sensor beam is clipped on the master polarizer level stage. Figure 28 shows an optomechanical model of the IR sensor beam layouts relative to the retarder level 1 and the polarizer level 2 .

There were two TCs that were installed in direct contact to the top of the QTR shown in Fig. 28(b). There are many TCs in the GOS enclosure itself. We used TC3 and TC4 taped directly onto the crystal quartz retarder substrate just outside the nominal illuminated aperture of the QTR. These TCs have response times better than $0.3 \mathrm{~s}$ and can operate to at least $175 \mathrm{deg}$ temperatures.

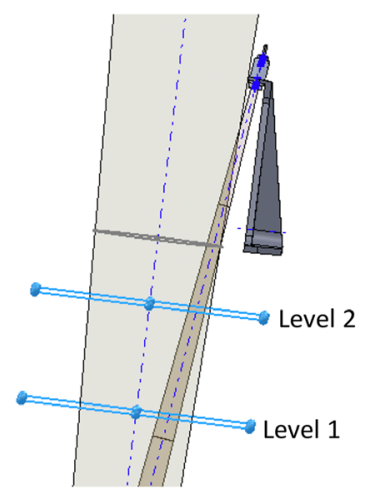

(a)

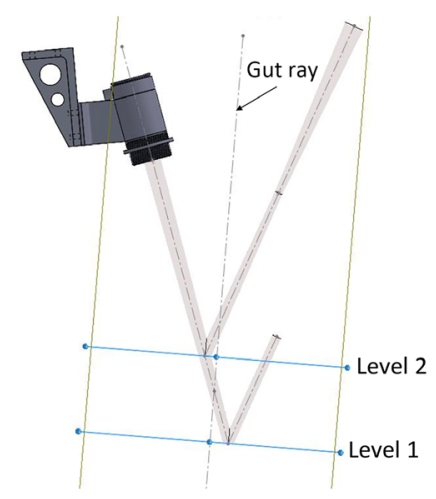

(b)

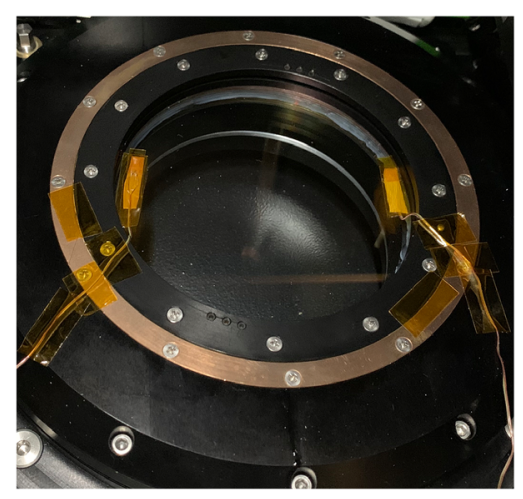

(c)

Fig. 28 (a) An optomechanical model of the IR1 sensor designed to measure temperatures for the polarizers on level 2 and for retarder centers on level 1. (b) The IR2 sensor which is well off-center on both levels. Sensors provide telescope operators with real-time temperature information and historical logging for data quality assurance. (c) Two TCs directly attached to the QTR mounted in level 1 above DKIST Gregorian focus used during on-Sun thermal testing. TCs were taped onto the optical surface just outside the optic clear aperture. 


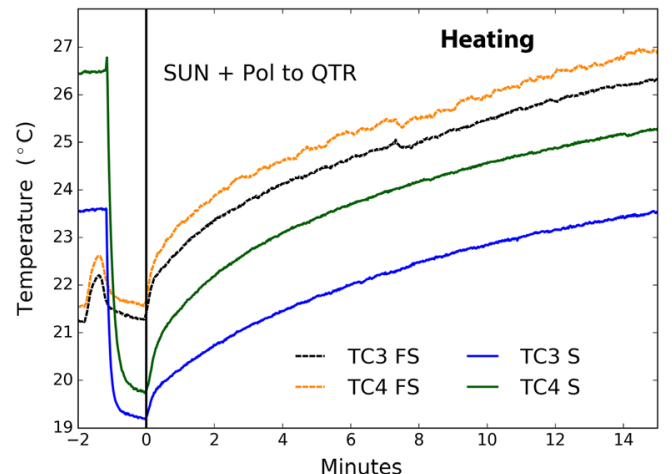

(a)

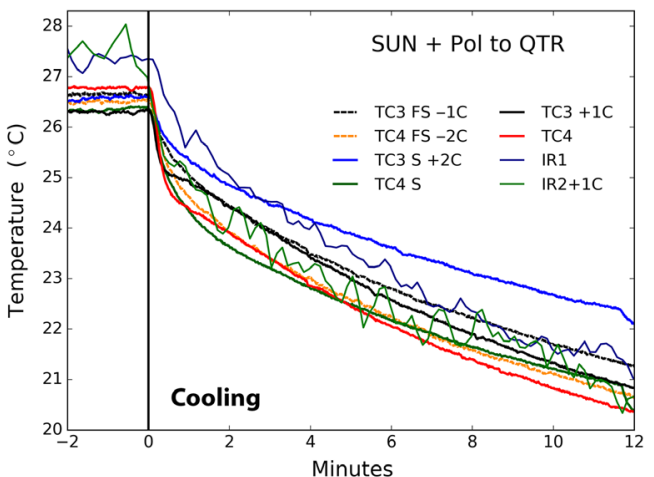

(b)

Fig. 29 (a) The TC temperatures for two separate tests over a 15-min span on the QTR. (b) Cooling of the QTR for three optic options on level 2 above: FS CalPol1 Spare polarizer denoted FSpol above, the Spol above, and no polarizer optic above.

The thermal testing was done on January 23 and 24, 2020. Observations were done with a photometric sky and winds in the 5- to 15-knot range, with the dome shutters open. On the morning of the 23, there were TCs taped on the uncoated substrate side of the FSpol called CalPoll outside the illuminated aperture directly touching the uncoated glass side. The optical configuration is for polarizing wires facing down toward DKIST M3, uncoated glass up toward M2 with the TC side having an easier vertical convection path. The QTR was installed below CalPol1 also with two TCs attached outside the illuminated aperture. These two optics (the CalPol1 Spare + QTR) were illuminated with the GOS ALS (Lamp) for roughly $2.5 \mathrm{~h}$.

The two solar illuminations of the QTR with the polarizers mounted in the beam give fairly similar thermal curves. Figure 29 shows the TCs data. Note no IR sensor data are available when the polarizers are in the beam ahead of the retarder. The optic starting temperatures were different between the two tests. The cool-down history is also different between both tests. The orange and black curves in Fig. 29 show the two TC data sets with the FSpol (CalPoll Spare) mounted ahead of the QTR. The blue and green curves show the QTR data but with a sapphire test polarizer inserted in the beam ahead of the QTR.

The solar illumination was stopped by closing the primary mirror (M1) covers. This process takes roughly $30 \mathrm{~s}$ as a linear stage moves covers across the mirror aperture. Figure 29(b) shows the cool-down rates of the QTR under all heating tests. The two TCs (TC3 and TC4) were measured when the QTR was used behind the CalPol1 Spare FSpol as the dashed black and dashed orange curves. The sapphire polarizer (Spol) was then used ahead of the QTR giving rise to the solid blue and green curves. Then no polarizer was used and the QTR saw the full 300-W beam. The solid black and red curves show the TCs. The significantly more noisy dark blue and dark green curves show the two IR diode sensors observing the front of the QTR.

The temperatures measured by the IR sensors were very similar between sapphire and FS substrate polarizers. Figure 30 shows the two illumination tests. The FS substrate results shown as solid curves while the sapphire substrate results are shown as dashed curves. The IR1 sensor is looking $14.2 \mathrm{~mm}$ off center of the polarizers and stabilized around $130^{\circ} \mathrm{C}$ for both polarizers within $3 \mathrm{~min}$. The IR2 sensor footprint is $43.3 \mathrm{~mm}$ off center near the edge of the illuminated portion of the optic. The TC for the FSpol shown as the red curve stabilized near $55^{\circ} \mathrm{C}$ and was mounted with a decenter near $58 \mathrm{~mm}$, near the edge of the $60-\mathrm{mm}$ radius of the FSpol substrate. We note that thermal models of the thin polarizer substrates do create strong temperature gradients from center to edge. Our heat absorption predictions suggest a few watts is absorbed through the aluminum wire reflection losses. The FSpol substrate is 1-mm thick while the sapphire substrate is only $0.7-\mathrm{mm}$ thick but with roughly 30 times increased conductivity.

\subsection{B.1 DKIST $\mathrm{MgF}_{2}$ Cryo SAR}

On January 24, we tested the three first-light DKIST CalRets in the full $300-\mathrm{W}$ beam. The CN SAR thermal models per HS18a ${ }^{63}$ suggest that this retarder should have absorbed flux at $0.5 \mathrm{~W}$ 
Harrington et al.: Polarization modeling and predictions for Daniel K. Inouye Solar Telescope, part 7...

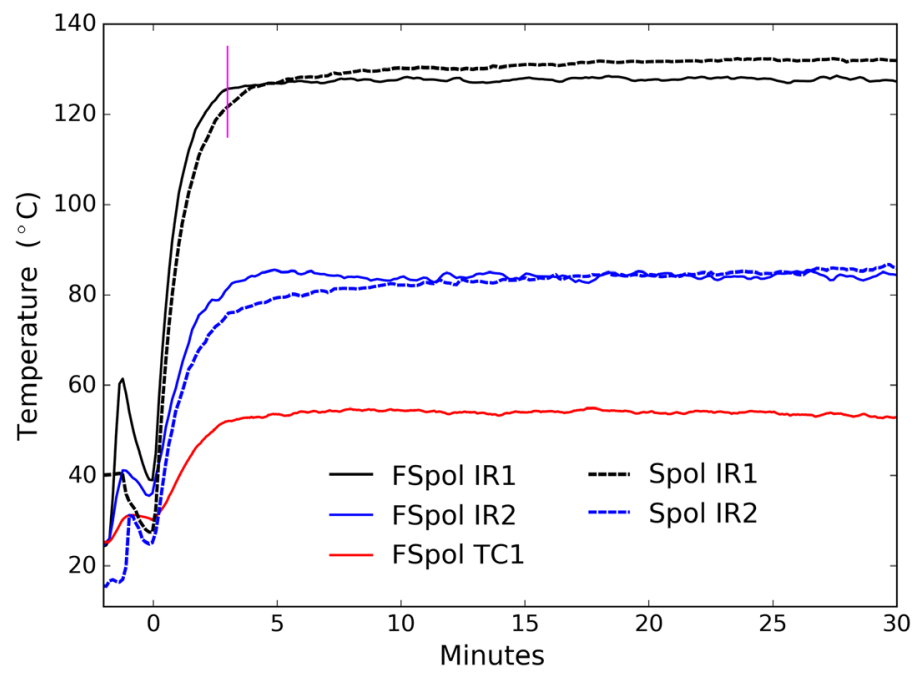

Fig. 30 Temperatures measured for the two polarizer types. Solid lines show the FSpol. Dashed lines show the Spol substrate. Both polarizers reached quite similar temperatures. Only the FSpol had a directly attached TC1.

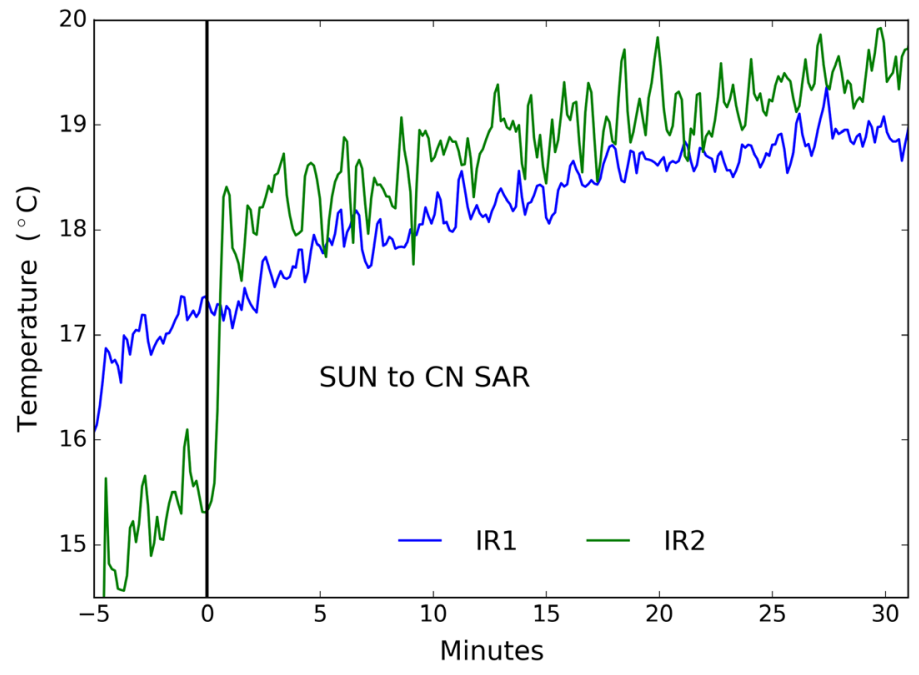

Fig. 31 Temperature measured for the $\mathrm{MgF}_{2}$ CalRet (Cryo SAR) during direct illumination in the $300-W$ beam. We note some mild influence likely from back-scatter off the 2.8 -arc min field aperture stop. The full 5-arc min field stop was not yet available for full $300-\mathrm{W}$ operations at the time of testing.

levels for the thermal model predictions at an airmass of 1 and the $\mathrm{MgF}_{2}$ crystals absorbing wavelengths longer than about $6000 \mathrm{~nm}$. The temperatures measured in Fig. 31 show a $1.5^{\circ} \mathrm{C}$ rise over 30 min of full illumination. The illumination begins with one IR sensor seeing a step in voltage as the green curve over a few seconds. The scattered light is different between these two IR sensors and the back reflected diffuse light from the 2.8-arc min field stop aperture at Gregorian focus nearby is an issue. Both sensors then track slow increases over the $30 \mathrm{~min}$ of illumination. We expect with the lower absorption that we measure a much smaller temperature change with this optic than with the QTR.

\subsection{B.2 DKIST Quartz Retarders}

The optical contact calibration retarder (OCret) and the ViSP SAR were tested sequentially. Figure 32 shows the $\sim 30 \mathrm{~min}$ heating period for both optics measured with the two IR diode 


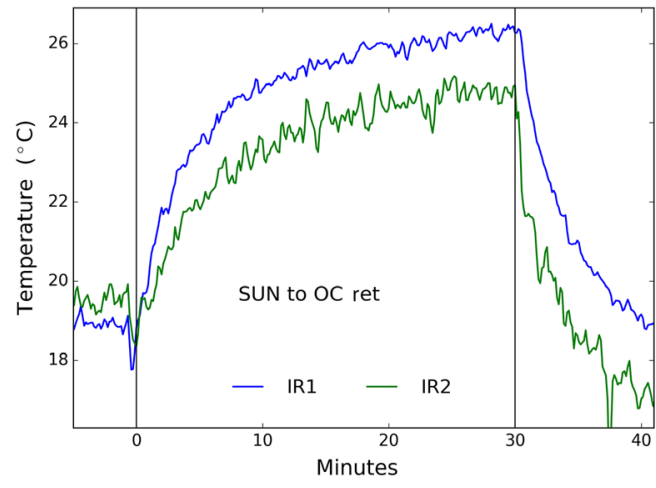

(a)

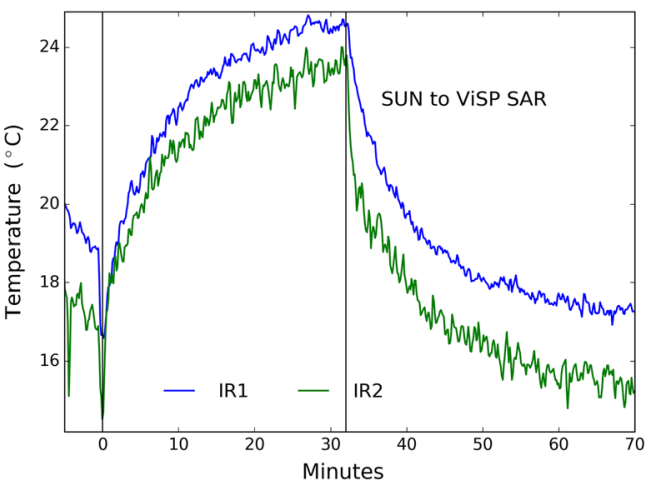

(b)

Fig. 32 (a) The optical contact retarder IR sensor temperatures and (b) the ViSP SAR. Both quartz retarders were illuminated in the full $300-\mathrm{W}$ beam without polarizers upstream.

sensors. Figure 32(a) shows the optical contact retarder, whereas Fig. 32(b) shows the ViSP SAR. The beginning and ending of illumination are noted with vertical solid black lines. The ViSP SAR had an extended cool-down period of over $30 \mathrm{~min}$, whereas the OCret was only measured during cooling for roughly 10 min before other testing commenced.

\subsection{B.3 Thermal Timescale Summary for Polarizers and Retarders}

We showed direct and remote temperature measurements for the DKIST retarders and polarizers in this section. The quartz-based retarders showed $6^{\circ} \mathrm{C}$ to $8^{\circ} \mathrm{C}$ temperature rise in the full $300-\mathrm{W}$ beam over $30 \mathrm{~min}$ illumination. Similar temperature rises were observed with both FS substrate and sapphire crystal substrate polarizers mounted ahead of the retarder. Retarder cool-down timescales were similar under all illumination scenarios with roughly $5^{\circ} \mathrm{C}$ drops in $15 \mathrm{~min}$. The MgF2 retarder does not absorb IR wavelengths and only rose $1.5^{\circ} \mathrm{C}$ in $30 \mathrm{~min}$ of direct exposure to the full $300-\mathrm{W}$ DKIST beam. The polarizers temperature response was very significantly faster with temperature rises of $90^{\circ} \mathrm{C}$ in just $3 \mathrm{~min}$, consistent with our preliminary thermal testing. We note that these polarizers are rated to thousands of hours of operation at temperatures of $250^{\circ} \mathrm{C}$ and maintain contrast at temperature. We confirm the strong radial gradient of temperature with the IR1 diode measuring close to center with temperatures $70^{\circ} \mathrm{C}$ above the TC outside the illuminated aperture but directly touching the polarizer substrate only $20^{\circ} \mathrm{C}$ above initial starting temperatures.

\section{Appendix C. Additional Mirror Coating Measurements for DKIST}

We show that metrology on coating witness samples can provide a very good initial system metrology model for astronomical telescopes provided the actual coating samples are directly measured. Many flavors of enhanced protected silver coatings have similar reflectivity, but the diattenuation and retardance terms vary substantially even for the same coating formula from the same vendor. We have procured more mirrors and assessed many more samples for DKIST since $\mathrm{H} 19^{67}$ was published. These include DKIST coudé lab installations and NCSP as well as optics internal to the various DKIST instruments (ViSP, DL-NIRSP, and CN). We show in this section measurements and updated models used in the system modeling. We also show the ellipsometric measurements covering 0.2 to $>35 \mu \mathrm{m}$ wavelengths along with comparisons to our own measurements.

\subsection{C.1 New Ellipsometric Measurements for DKIST Mirror Groups}

Figure 33 shows new ellipsometric measurements from Woollam. The equipment was unable to measure polarization at incidence angles at below $15 \mathrm{deg}$. Data were recorded from $15 \mathrm{deg}$ to 


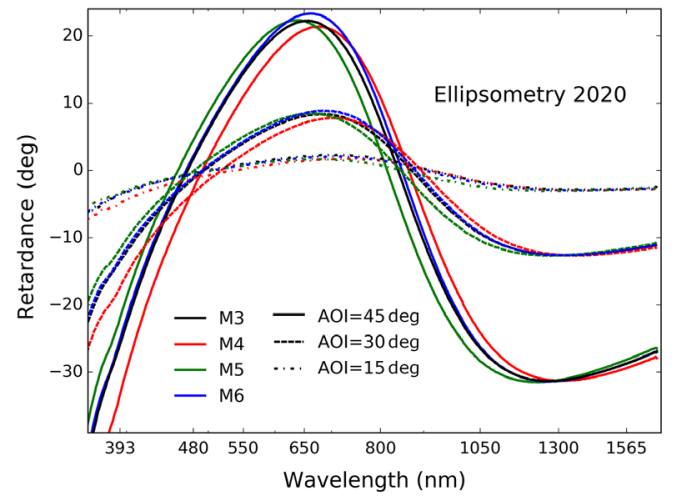

(a)

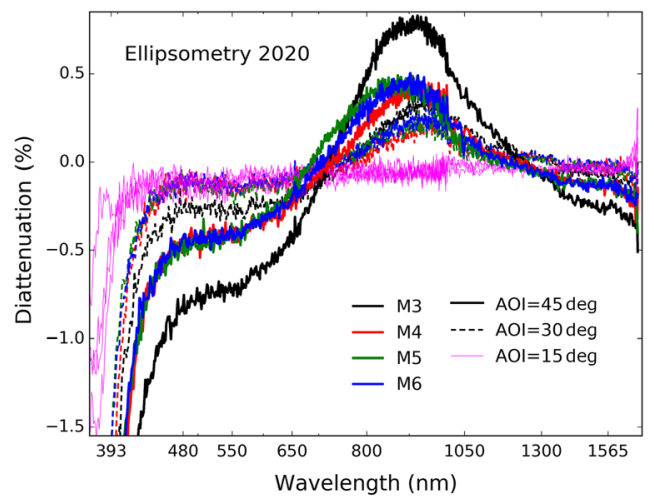

(b)

Fig. 33 (a) Retardance and (b) diattenuation derived from the new ellipsometry data for these particular mirror coating witness samples (DKIST M3 to M6) covering incidence angles of $15 \mathrm{deg}$, $30 \mathrm{deg}$, and 45 deg.

either $60 \mathrm{deg}$ or $75 \mathrm{deg}$ in steps of $5 \mathrm{deg}$. We see similar results to our internal metrology system in $\mathrm{H}_{1} 9^{67}$ at 45 -deg incidence. Figure 33(a) shows retardance derived from the Mueller matrix ellipsometry on the M3, M4, M5, and M6 coating witness samples. Figure 33(b) shows diattenuation. We only show incidence angles of $15 \mathrm{deg}, 30 \mathrm{deg}$, and $45 \mathrm{deg}$ as representative of the range for the DKIST mirror groups used in the main text.

\subsection{C.2 Extension of the DKIST Coating Models to the Infrared}

DKIST has an operational requirement to deliver an optical beam covering wavelengths at least as long as $28 \mu \mathrm{m}$. For the first-light instrument suite, the $\mathrm{CN}$ has filters at wavelengths significantly longer than the 1.6- $\mu \mathrm{m}$ cut off in our NCSP metrology tool. Existing CN filters include spectral lines of Mg VIII at $3.03 \mu \mathrm{m}$, Si IX at $3.94 \mu \mathrm{m}$, and CO at $4.65 \mu \mathrm{m}$. We have confidence that the metrology model presented here can be easily extended to longer wavelengths. We also do not anticipate any greatly different system behavior given the magnitude of polarization spectral features measured in these samples.

We show ellipsometric measurements at an incidence angle of 45 deg in Fig. 34 for the nominal DKIST enhanced protected silver used in mirrors 2 to 7 , many of the DL-NIRSP

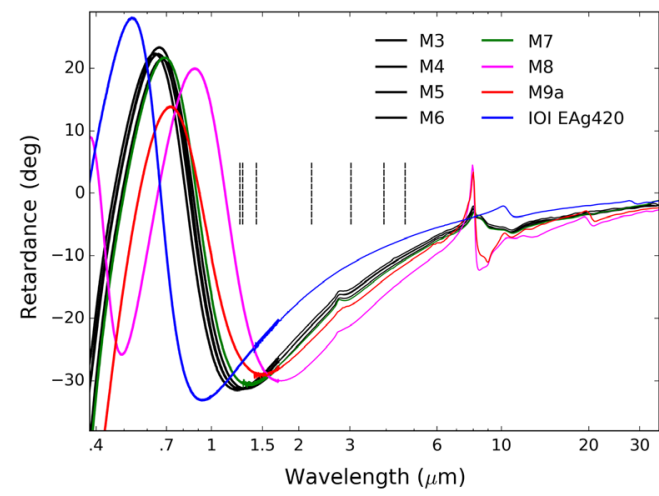

(a)

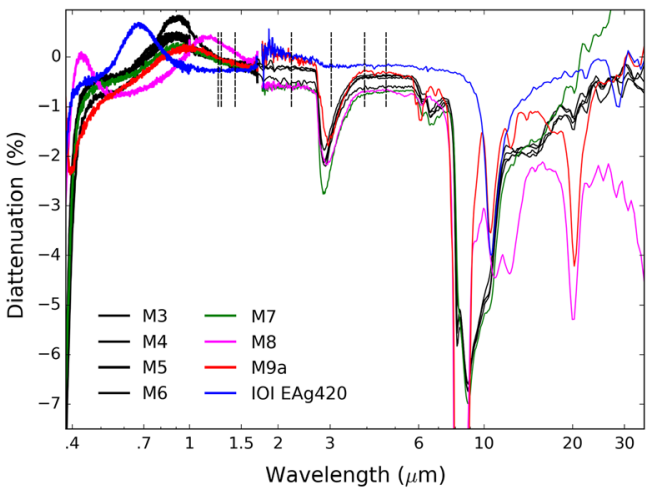

(b)

Fig. 34 (a) The retardance and (b) diattenuation measured on coating witness samples at an incidence angle of $45 \mathrm{deg}$ for the many layer protected silver coating used on DKIST mirrors M3, M4, M5, and M6. We also include mirrors combined to modulation matrices with M7, M8, $\mathrm{M} 9 \mathrm{a}$, and the IOI formulation used on DKIST M9 as well as some ViSP and NCSP mirrors. Vertical dashed black lines show wavelengths for the current infrared $\mathrm{CN}$ instrument filter set. 
instrument mirrors, and all of the $\mathrm{CN}$ instrument mirrors. We note that we have measurements covering a range of incidence angles from 30 deg to 60 deg on many samples to inform future calibration models derived in the IR. Given the success of fitting the system model parameters presented here, we conclude that will be straightforward to extend a few mirror polarization terms to the infrared.

\subsection{C.3 DKIST M9a and DL-FM1: Zygo Standard Protected Silver}

We show few layer protected silver-coated mirrors from Zygo in Fig. 35. The DKIST coudé mirror M9a as well as the DL-NIRSP first-fold mirror (DL-FM1) were both coated with this style silver. The NLSP measurements in September 2019 show that the DKIST optic coatings are significantly different than the model and the historical sample. However, the coating formula, reflectivity, and spectral smoothness are as expected.

We also compare NLSP diattenuation with the $S$ and $P$ reflectivity measurements from Zygo during optic acceptance testing. Note that the reflected diattenuation and in-band reflectivity for DL-FM1 and M9a measured by Zygo was already published in H19, ${ }^{67}$ Appendix C.5, Fig. 60. The diattenuation in $\mathrm{H} 19^{67}$ incorrectly included a factor of 0.5 in the computation of the diattenuation following the definition as the normalized reflectivity difference. The Zygo metrology has been corrected here and agrees within better than $0.5 \%$ to NLSP measurements with very similar spectral behavior.

\subsection{C.4 DKIST M8: EMF AG99 Protected Silver and Comparison with DL F0O-201}

Evaporated metal films (EMF) coated the eighth DKIST system mirror M8 in the coudé laboratory. We show NLSP reflected retardance and diattenuation at 45-deg incidence from many layer protected silver-coated mirror samples in Fig. 36. Note that unpolarized reflectivity of these EMF samples at 8-deg incidence angle was published in $\mathrm{H} 19^{67}$ Appendix C.8 and Fig. 63. The M8 off axis parabola chief ray is incident at roughly $5 \mathrm{deg}$. The magenta curves show a nominal vendor model prediction before M8 was actually coated. We show the DL-NIRSP mirror F00201 coated by EMF as well, though not necessarily with the same formula and over a year earlier. The two coatings are significantly different in polarization response. Even though both coatings are of the AG99 coating family, the exact spectral behavior is significantly different. As all curves are spectrally smooth, and the diattenuation magnitude is $<2 \%$, this coating family should have little impact on DKIST system calibrations.

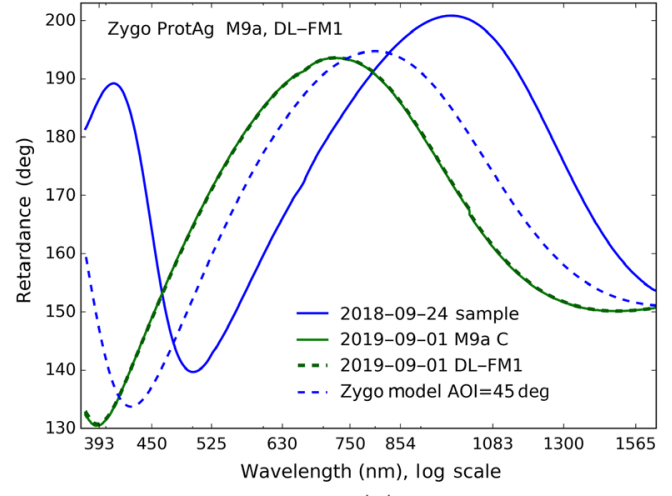

(a)

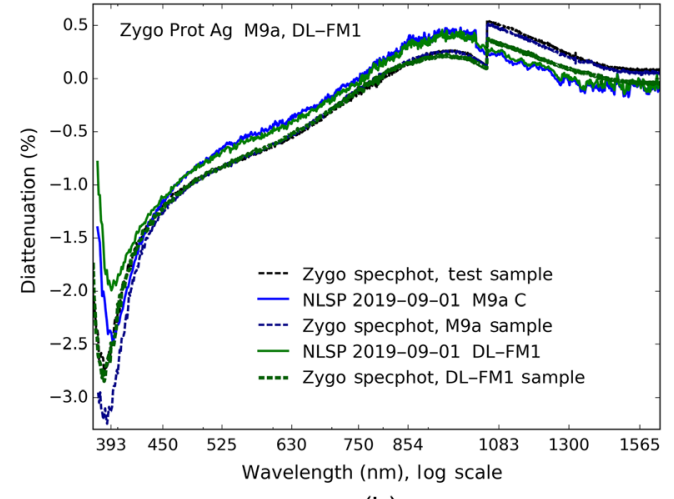

(b)

Fig. 35 (a) The retardance and (b) diattenuation derived at an incidence angle of 45 deg for a many layer protected silver coating from the commercial vendor Zygo used on DKIST mirror M9a and DL-NIRSP fold mirror 1 (DL-FM1). 


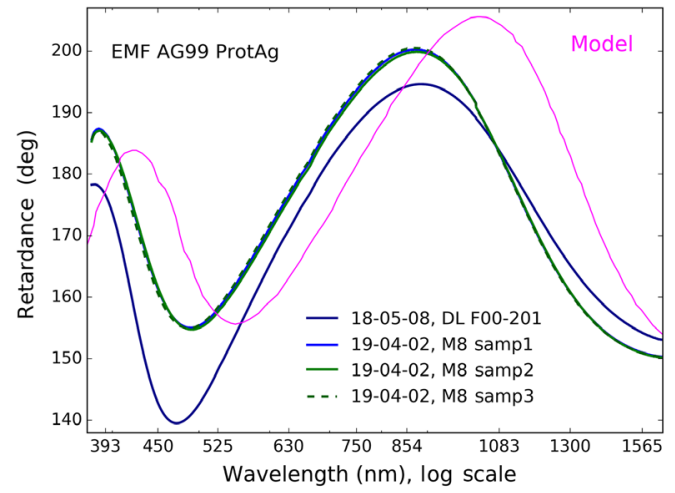

(a)

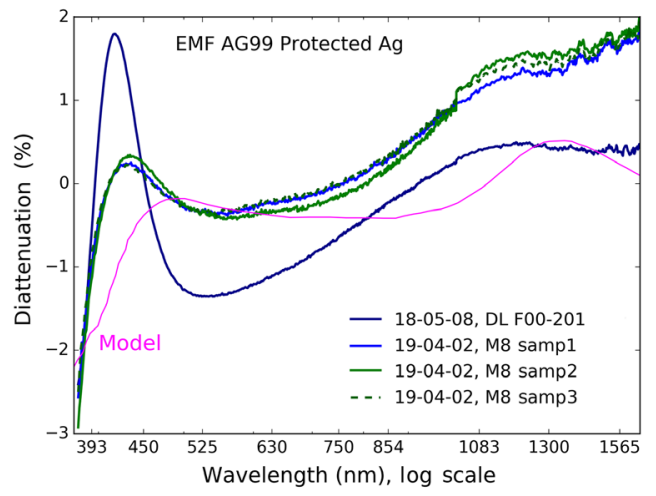

(b)

Fig. 36 (a) The retardance and (b) diattenuation derived at an incidence angle of 45 deg for samples for a many layer protected silver coating from the commercial vendor Dynasil EMF. The three DKIST M8 samples are compared with the DL-NIRSP spectrograph optic F00-201. The vendor model for the coating family is shown in magenta.

\subsection{C.5 NCSP F1: IOI coating on Dream Elliptical 6" Flats and ViSP Fold and Feed}

An Infinite Optics Inc. (IOI) enhanced protected silver coating with run 6-8065 was shot in December 2019 using the EAg1-420 formula. The same formula was previously used on the first- and second-ViSP feed mirrors. These mirrors were stripped and recoated after the results published in $\mathrm{H} 19^{67}$ after some mild scuffing was discovered inside the clear aperture. To achieve uniformity and consistency on the relatively large ViSP optics, IOI performed two separate coating runs. Coating run 6-7879 contained the ViSP fold mirror 1, denoted F1. Coating run 6-7881 contained the ViSP feed mirror 1, denoted FM1. In Fig. 37, we show the IOI reflectance measurements at $8 \mathrm{deg}$ for the EAg-420 two-layer protected silver coatings deposited on the ViSP instrument feed optics and NCSP fold mirror 1. The ViSP FM1 is shown in green while F1 is shown in blue. In Fig. 37(a), the wide UV to IR bandpass is shown. In Fig. 37(b), the nominal visible to NIR bandpass is highlighted showing reflectivity always above $96.5 \%$ with good spectral smoothness, unlike the original coating data shown in H19. ${ }^{67}$

We procured four witness samples for our lab polarization testing with NLSP. We show the NLSP reflected retardance and diattenuation at 45-deg incidence for the EAg1-420 two-layer

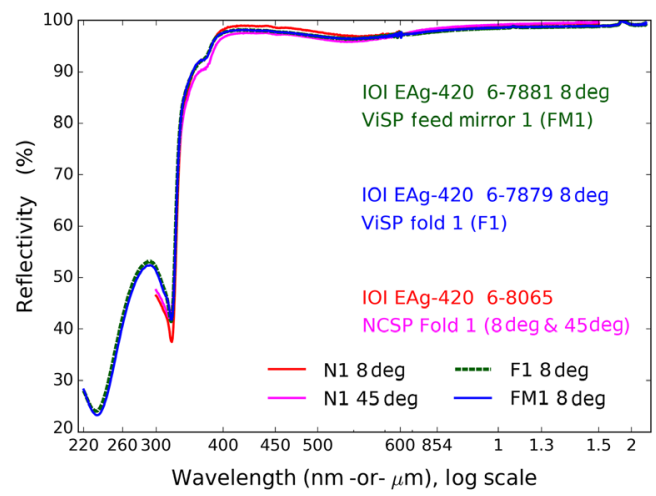

(a)

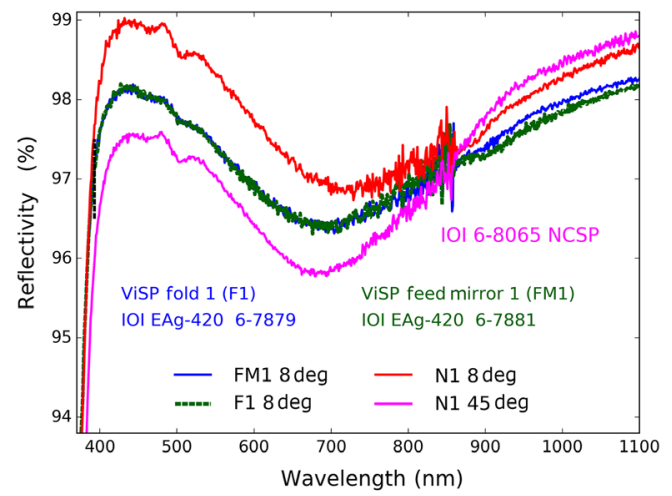

(b)

Fig. 37 The reflectivity measured at 8-deg incidence angle by $10 \mathrm{I}$ for the stripped and recoated ViSP instrument feed mirrors. (a) The full 220- to 2500-nm bandpass measured at IOI. The FM1 coating is shown in green, whereas the F1 coating is shown in blue. (b) The ViSP nominal bandpass with reflectivity over $96.5 \%$. The vertical black line shows $97 \%$ reflectivity at $393 \mathrm{~nm}$ wavelength. 


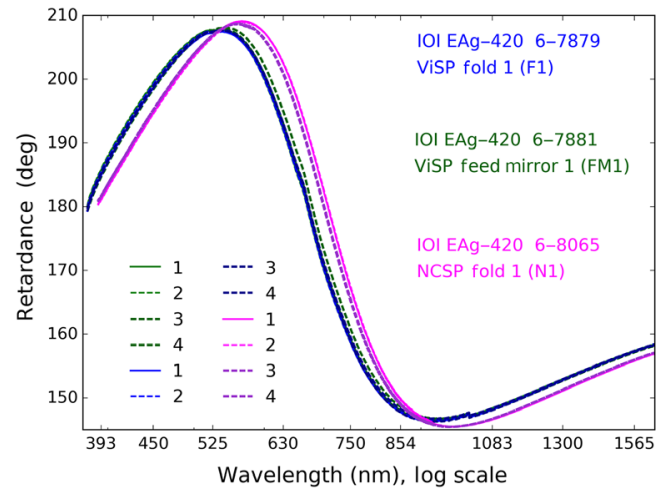

(a)

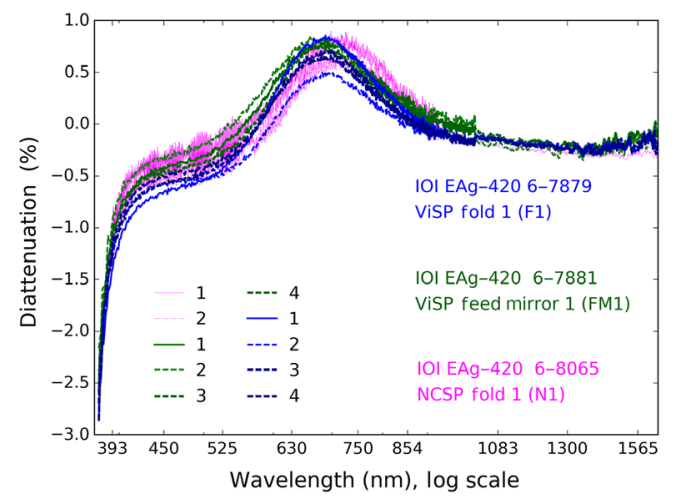

(b)

Fig. 38 (a) The retardance and (b) diattenuation derived at an incidence angle of 45 deg for a few layer protected silver coating (EAg-420) from the commercial vendor IOI. This coating was used on the ViSP instrument feed mirror 1 (FM1) shown in green, and fold mirror 1 (F1) shown in blue. We also used this coating but from shot 6-8065 on the NCSP elliptical fold mirror 1 shown in magenta.

protected silver formula in Fig. 38. We tested each of the four samples for each coating run independently and label them numerically. The graphics color shows NCSP fold 1 (N1) as shades of magenta, ViSP F1 as shades of blue, and ViSP FM1 as shades of green. All samples are very consistent in retardance. The diattenuation varies by up to $0.5 \%$ but there are known systematic errors in the NLSP diattenuation measurements of this magnitude in addition to the incidence angle variation with mounting of samples. However, the spectral shapes of the samples are all very consistent with diattenuation magnitudes below $1 \%$ for all but the shortest wavelengths.

\subsection{C.6 NCSP F2 and F4: Edmund Optics Protected Silver 2" and 6" Flats}

The NCSP second-fold mirror is a 6-in.-diameter Edmund Optics protected silver-coated fold at an incidence angle of $45 \mathrm{deg}$. The fourth NCSP fold is also the same formula purchased at the same time. We show NLSP measurements of protected silver-coated mirrors from Edmund Optics in Fig. 39 at an incidence angle of $45 \mathrm{deg}$. We note that we also measured a 6-in.-diameter mirror used in the DKIST VBI as well as four separate 1-in.-diameter samples.

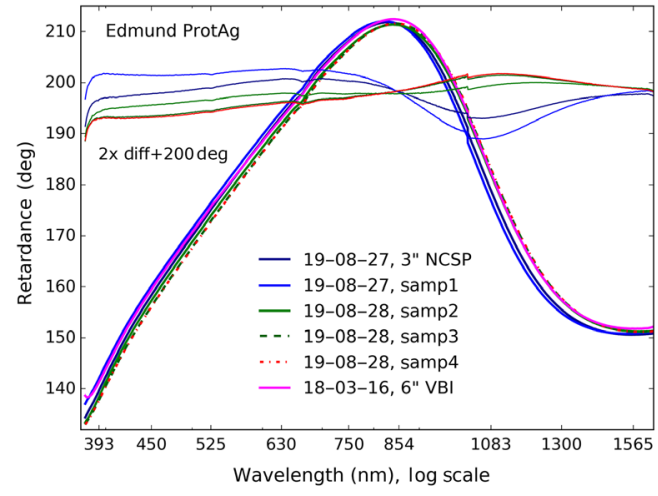

(a)

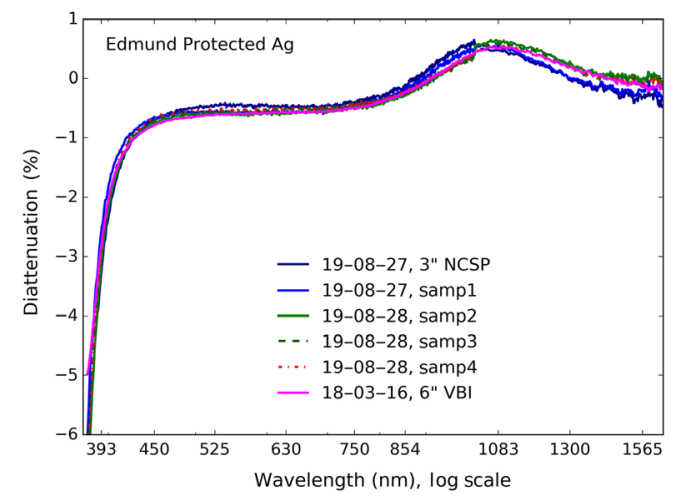

(b)

Fig. 39 (a) The retardance and (b) diattenuation for an incidence angle of 45 deg derived for a standard protected silver coating from the commercial vendor Edmund Optics. We show measurements on a large VBI instrument mirror, the actual 3 in. NCSP mirror and four separate 1 in.-diameter samples These Edmund coatings have been very stable and repeatable. We show in (a) the retardance difference multiplied by 2 and shifted up by 200 deg for clarity. 


\subsection{C.7 NCSP F3: Thor Labs Protected Silver 3" Flat}

We show protected silver coated mirrors from Thor Labs in Fig. 40. The 2-in.-diameter NCSP fold mirror was directly measured and is shown in blue. This NCSP mirror coating is substantially different from the same catalog number mirrors bought at 1-in. diameter and tested for $\mathrm{H} 19^{67}$ shown in green. No guarantee is made by most vendors about coating polarization or repeatability so this is not unexpected. The wavelength shift of the retardance and diattenuation peaks suggest the dielectric coating layers are roughly half as thick.

\subsection{C.8 NCSP System Model for 10 Combined Mirrors and Modulator}

The system model and modulation matrix for NCSP requires estimates of the polarization properties for the mirrors M7 through the modulator. We replicate the M7 properties from measurements presented in $\mathrm{H}_{1} 9^{67}$ as well as presenting new metrology here. In Table 8, we outline the mirrors and a representative coating model for the NCSP feed optics. In the

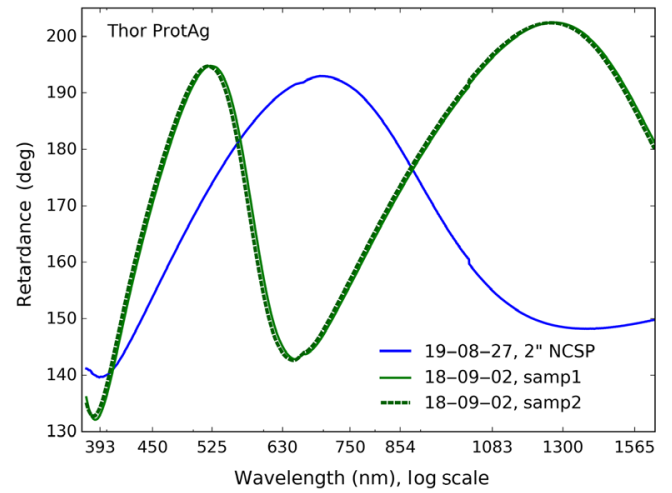

(a)

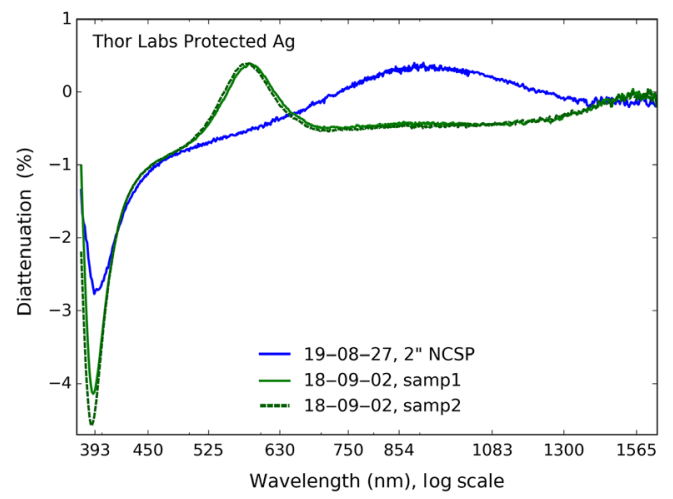

(b)

Fig. 40 (a) The retardance and (b) diattenuation derived at an incidence angle of 45 deg for a standard protected silver coating from the commercial vendor Thor Labs. We note significant change between the NCSP fold mirror from 2019 shown in blue and the two samples shown in green that were purchased and tested a few years ago.

Table 8 NCSP mirrors and one- or two-layer coating models.

\begin{tabular}{|c|c|c|c|c|}
\hline Opt & $\mathrm{AOI}$ & Coat & Fold & Formula \\
\hline M7 & 45 & DEAg & $Y$ & $4.8 \mathrm{ZnS}, 119 \mathrm{Al}_{2} \mathrm{O}_{3}$ \\
\hline M8 & 5.4 & EMF & $x$ & Ignore \\
\hline M9 & 10 & IOI EAg450 & $x$ & $30 \mathrm{ZnS}, 30 \mathrm{Al}_{2} \mathrm{O}_{3}$ \\
\hline M9a & 9 & Zygo & $x$ & $140 \mathrm{Al}_{2} \mathrm{O}_{3}$ \\
\hline CNscn & 4 & DEAg 16BB07 & $x$ & Ignore \\
\hline CNfoc & 1.1 & DEAg 16BB21 & $x$ & Ignore \\
\hline NCSP 1 & 45 & IOI EAg420 & $Y$ & 55-nm ZnS \\
\hline NCSP 2 & 45 & Edmund PAg & $Y$ and $c 90 X$ & 100-nm ZnS \\
\hline NCSP 3 & 10 & Edmund PAg & $x$ & $100-\mathrm{nm} Z \mathrm{nS}$ \\
\hline NCSP 4 & 10 & Thor PAg & $x$ & $165-\mathrm{nm} \mathrm{SiO} 2$ \\
\hline
\end{tabular}


first column, we show the optic name. In the second column [angle of incidence (AOI)], we show the incidence angle. In the third column (coat), we name the coating vendor and formula if available. In the fourth column (fold), we list the orientation of the fold axis for the mirror. Most mirrors are in simple horizontal $(X)$ or vertical $(Y)$ fold configurations. The second fold mirror NCSP FM2 folds the beam down $(Y)$ but also is clocked 90 deg horizontally to the beam (c90X). The fifth column (formula) of Table 8 shows the coating formula model used for the dielectric layers.

For DKIST M8, the EMF coating can be ignored at an incidence angle of 5.4 deg. DKIST M9 is a flavor of the IOI protected silver coating we model as two layers. The removable DKIST pickoff mirror M9a was coated by Zygo with their standard silver. The two $\mathrm{CN}$ feed mirrors are at very low incidence angle and we ignore them for now, though the formula is the standard DKIST enhanced silver with coating runs listed above and coating models published in H19. ${ }^{67}$ The NCSP mirrors were commercially procured and are modeled with single-layer coatings. We note that the first two mirrors are used at 45-deg incidence and we use the measured data directly for those two mirrors. We require the coating model to extrapolate to incidence angles without measurements.

Figure 41 shows the individual mirror models and associated data used in the NCSP system model. Figure 41(a) shows retardance. Figure 41(b) shows diattenuation. The dashed lines show our NLSP metrology data at 45-deg incidence angle, whereas the solid lines show the Berreman coating model from Table 8 .

The NCSP modulator was designed as a five-layer achromat for efficiency over a very broad wavelength range. It was fabricated in the same batch as the DL-NIRSP polycarbonate modulator upgrade (see $\mathrm{H} 20^{80}$ Appendix E for metrology and retarder design details). We note that the measured beam deflection is 2.11 arc se for this optic at $630 \mathrm{~nm}$ wavelength. This deflection is incredibly small due to a new active deflection nulling fabrication technique deployed by Meadowlark Optics. We achieved 0.08 waves peak to valley transmitted wavefront error with most of that error as the power term. The modulation efficiency for just the retarder was shown in $\mathrm{H} 20^{80}$ Fig. 42. Calculations including this system model does not modify the $Q U V$ efficiency balance above or below the same balance thresholds previously published. Spatial variation of elliptical retardance was shown in $\mathrm{H} 20^{80}$ (Figs. 43-45) with only a few degrees spatial variation anticipated across the 3-mm beam footprint in NCSP with centering of the beam of better than $2 \mathrm{~mm}$. The retardance sensitivity to tilt angle was measured for this retarder in $\mathrm{H} 20^{80} \mathrm{Fig}$. 46 to be $<1$ deg as a function of wavelength per retardance component though the total magnitude is in the range $100 \mathrm{deg}$ to $140 \mathrm{deg}$ over the bandpass.

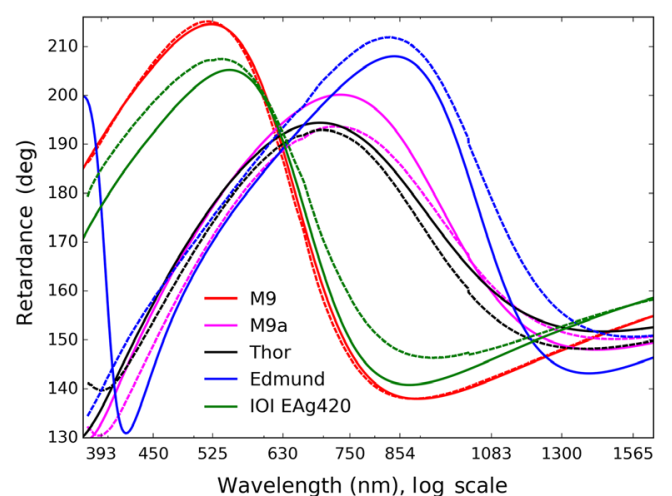

(a)

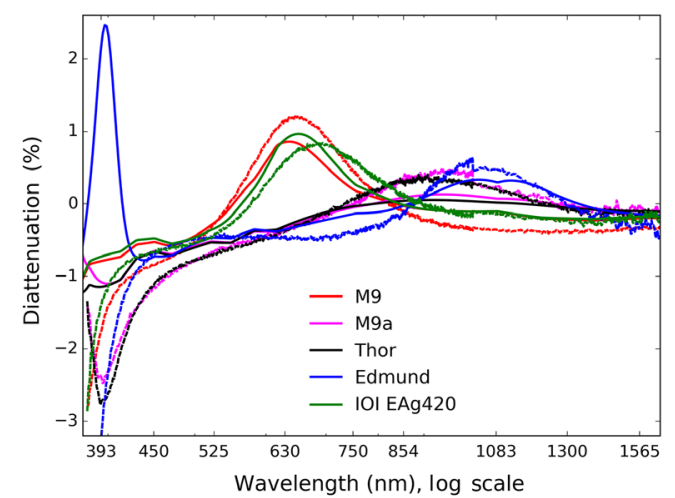

(b)

Fig. 41 The new NCSP mirror measurements and single-layer model coating models from Table 8 leading into the NCSP system model. Solid lines show the Berreman coating model. Dashed shows the NLSP data on witness samples at 45-deg incidence angle. (a) retardance and (b) diattenuation. Red shows the M9 coating from IOI. Magenta shows the Zygo coating on M9a. Black shows the Thor Labs coating on NCSP fold 4. Blue shows the Edmund Optics coating on NCSP folds 2 and 3 . Green shows an IOI coating on the NCSP fold 1. We note a very bad mismatch in diattenuation between model and data for the Edmund coating. 


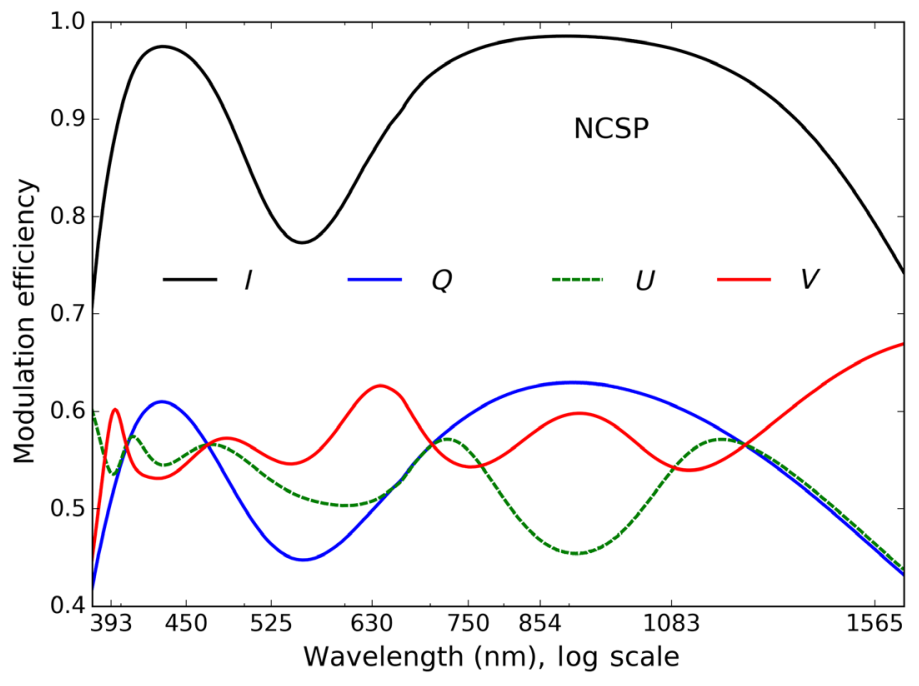

Fig. 42 NCSP system modulation efficiency model from M7 through the NCSP modulator included. Colors show efficiency for different Stokes parameters.

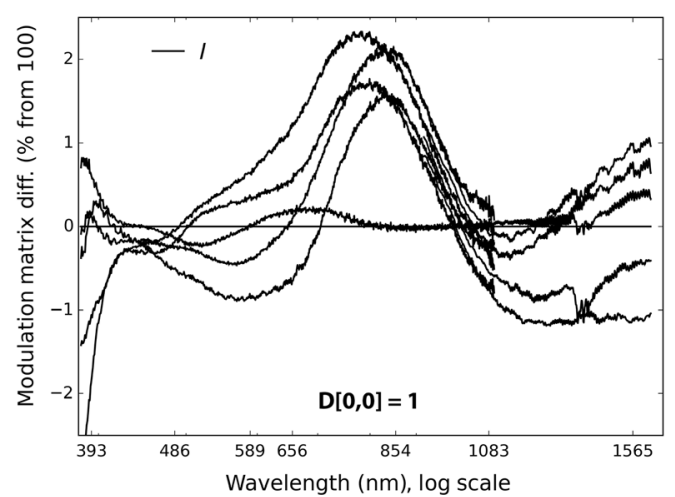

(a)

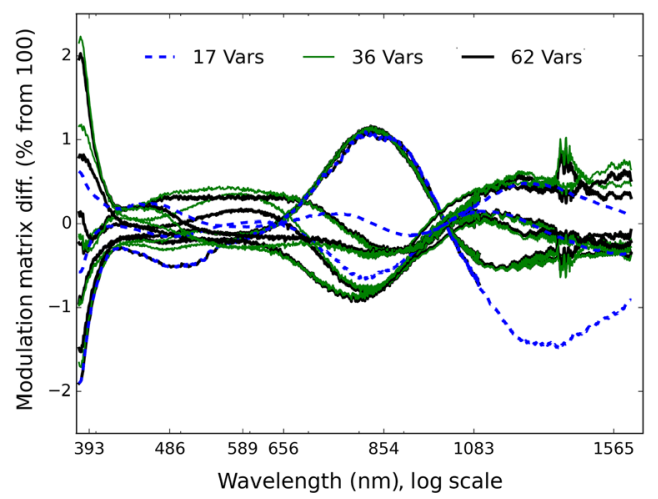

(b)

Fig. 43 (a) The five free modulation matrix intensity elements (first column) when doing the 137variable DKIST module fit with the $[0,0]$ element forced to 1. (b) The 17, 36, and 62 variable model intensity modulation terms where we used our own routines leaving all modulation matrix elements as free parameters. The 17-variable model in dashed blue and the 36-variable model in green had no free parameters for $l_{\text {sys }}$. The 62 -variable model in black had the additional $26 l_{\text {sys }}$ variables.

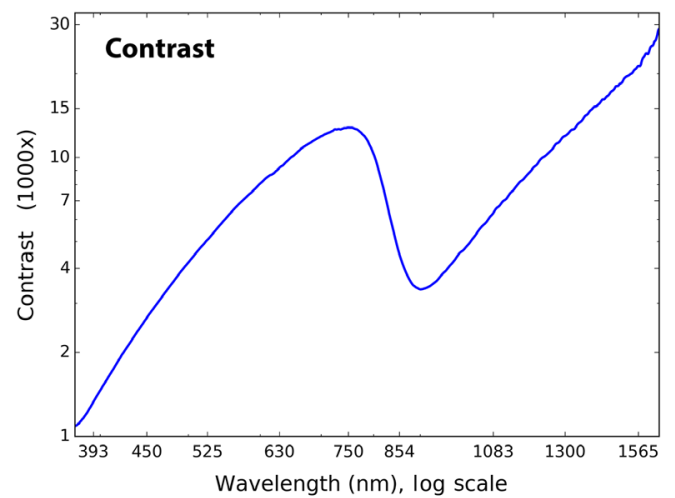

(a)

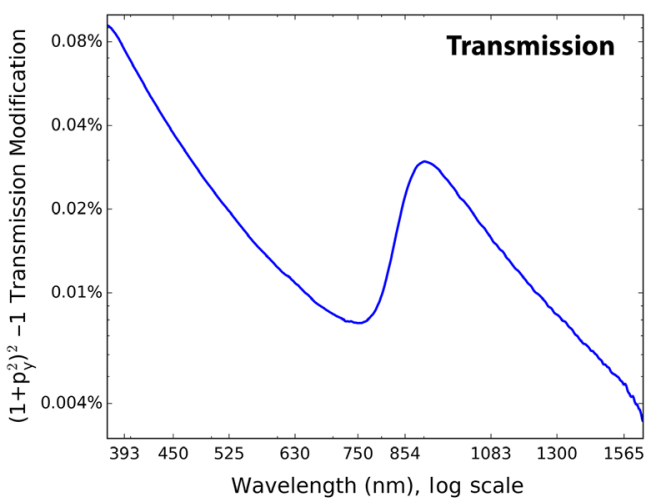

(b)

Fig. 44 (a) the polarizer contrast ratio following the $p_{y}$ lookup table currently used in DKIST calibration. (b) The transmission function modification term $\left(1+p_{y}^{2}\right)^{2}$ due to leakage in the perpendicular polarization state. 


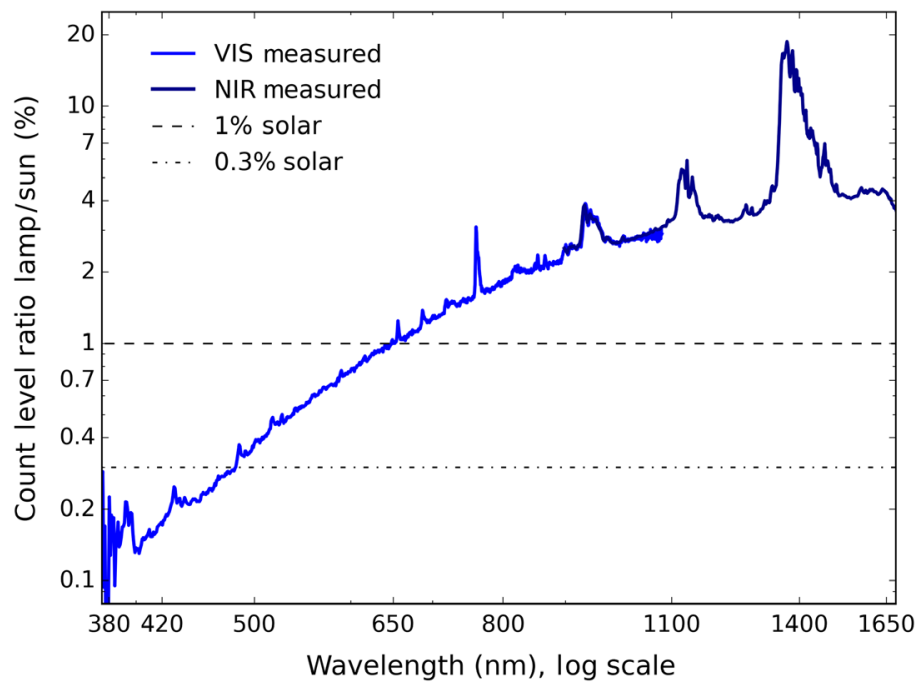

Fig. 45 The ratio of detected counts lamp divided by solar. The VIS spectrograph is shown in blue, whereas the NIR spectrograph is shown in dark blue. We show horizontal lines representing $0.3 \%$ and $1.0 \%$ ratios for reference.

\section{Appendix D. Mueller Matrix Conventions}

We summarize here the Mueller matrix terms and conventions for relating reflectivity and retardance to Mueller matrix elements outlined in several references. ${ }^{82,83,88}$

\subsection{D.1 Formalisms: $\left(R_{s}, R_{p}, \delta\right)$ or $(X, \tau)$}

Many solar telescopes perform calibrations following an $(X, \tau)$ style Mueller matrix, where the $X$ term relates to diattenuation and $\tau$ is retardance. ${ }^{58,59,91,103-106}$ We reproduce here how to relate this solar telescope formalism to a reflectivity and phase formalism more common in physical optics. Switching between conventions allows us to compare reflectivity, diattenuation, and retardance: $82,83,88$

$$
\mathbf{M}_{i j}=\left(\begin{array}{cccc}
1 & \Delta & 0 & 0 \\
\Delta & 1 & 0 & 0 \\
0 & 0 & \frac{\sqrt{R_{p} R_{s}}}{R_{\text {avg }}} C_{\delta} & \frac{\sqrt{R_{p} R_{s}}}{R_{\text {avg }}} S_{\delta} \\
0 & 0 & -\frac{\sqrt{R_{p} R_{s}}}{R_{\text {avg }}} S_{\delta} & \frac{\sqrt{R_{p} R_{s}}}{R_{\text {avg }}} C_{\delta}
\end{array}\right) .
$$

We adopt a standard notation, where the $S$ and $P$ polarization states represent incoming linear polarization states parallel and perpendicular to the plane of incidence. Their reflectivity is denoted as $R_{s}$ and $R_{p}$, respectively, and their average is denoted as $R_{\text {avg }}$. Retardance is denoted as $\delta$ which has the same meaning as $\tau$ in the common solar telescope calibration convention. In Eq. (12), we show a common definition of the Mueller matrix for a mirror folded along the $+Q$ plane. The $I I$ element is the average of $S$ and $P$ linear polarization state reflectivities. The retardance $(\delta)$ is a term in the sine and cosine functions in the $U V$ rotation matrix of the lower right quadrant. We have abbreviated these terms as $C_{\delta}$ and $S_{\delta}$. In the normalized Mueller matrix, the $\frac{I Q}{I I}$ and $\frac{Q I}{I I}$ terms are a normalized reflectivity difference ratio $\left(R_{s}-R_{p}\right) /\left(R_{s}+R_{p}\right)$ often denoted with a capital delta $(\Delta)$. The lower right $U V$ rotation matrix terms are modified by the scale factor $\frac{\sqrt{R_{p} R_{s}}}{R_{\text {avg }}}$. This term is above 0.999 for mirrors with diattenuation $<10 \%$ as are all mirrors considered for DKIST in their appropriate bandpass of use with the instruments: 


$$
\mathbf{M}_{i j}=\frac{R_{s}\left(1+X^{2}\right)}{2}\left(\begin{array}{cccc}
1 & \frac{1-X^{2}}{1+X^{2}} & 0 & 0 \\
\frac{1-X^{2}}{1+X^{2}} & 1 & 0 & 0 \\
0 & 0 & \frac{2 X}{1+X^{2}} C_{\tau} & \frac{2 X}{1+X^{2}} S_{\tau} \\
0 & 0 & \frac{-2 X}{1+X^{2}} S_{\tau} & \frac{2 X}{1+X^{2}} C_{\tau}
\end{array}\right) .
$$

A reflectivity ratio denoted $X$ is defined as $X=\sqrt{\frac{R_{p}}{R_{s}}}$. This can be computed from the $I Q$ or $Q I$ elements of the intensity-normalized Mueller matrix $(I Q / I I$ or $Q I / I I)$ and is typically near 1. We divide out one of the polarized reflectivities and denote the upper $2 \times 2$ submatrix in terms of this intensity reflection coefficient $X$. A normalized convention for the Mueller matrix with a total transmission term outside the matrix and $I I=1$ is shown in Eq. (13). The overall throughput term $I I$ is scaled by $\frac{R_{s}\left(1+X^{2}\right)}{2}$ for every matrix in our group model. As diattenuation is low, $X \sim 1$ and thus the overall throughput is close to 1 . The retardance is denoted as tau $(\tau)$ in this matrix following the solar telescope notation convention. Other systems using this formalism include The advanced Stokes polarimeter at the DST, ${ }^{11}$ The Hida Domeless Solar Telescope, ${ }^{103-105}$ the German Vacuum Tower Telescope ${ }^{58}$ the Polarimetric Littrow Spectrograph, ${ }^{59}$ and the solar tower in Arcetri: ${ }^{106}$

$$
\begin{gathered}
\frac{I Q}{I I}=\Delta=\frac{1-X^{2}}{1+X^{2}}=\frac{R_{s}-R_{p}}{R_{s}+R_{p}}, \\
X^{2}=\frac{1-\Delta}{1+\Delta} .
\end{gathered}
$$

We also can solve for $X, \Delta$, and reflectivities using the terms in Eqs. (12) and (13). The relations between $(X, \tau)$ and optical properties such as throughput and polarized reflectivities $R_{s}$ and $R_{p}$ are useful when comparing PolCals and throughput estimates from these varying conventions. We convert the normalized [1,0] and [0,1] matrix elements denoted as $\Delta$ to $X$ and polarized reflectivity terms in Eq. (14). We also show an equation for $X$ in terms of diattenuation $(\Delta)$ in Eq. (15).

\subsection{D.2 Rotation Conventions}

$$
\mathbb{R}_{\theta}=\left(\begin{array}{cccc}
1 & 0 & 0 & 0 \\
0 & \cos (2 \theta) & \sin (2 \theta) & 0 \\
0 & -\sin (2 \theta) & \cos (2 \theta) & 0 \\
0 & 0 & 0 & 1
\end{array}\right)
$$

We follow the rotation convention for polarimetry that angles are multiplied by 2 for rotation of the Stokes parameters with the sign convention in Eq. (16). We rotate the Mueller matrix of the GOS optics into the local coordinates of the beam at that optical station following the standard transform.

\subsection{D.3 $I_{\text {sys }}$ and Intensity Modulation Fits: Comparing Normalized Outputs}

Intensity normalization of the modulation matrix can be done with a few techniques. The DKIST algorithms currently fit all modulation matrix elements separately while forcing the $[0,0]$ element to be 1 . Alternate options used by some include normalizing the entire modulation matrix by the maximum term in the first column with this pseudocode: MAX(modmat[:,0]). Other algorithms, including our 17,36, and 62-variable fits let the intensity modulation terms in the first column of the matrix be free parameters anticipating some small coupling between system throughput variables $\left(I_{\text {sys }}\right)$ and the intensity modulation term magnitudes. Figure 43 shows the 137 -variable 
model fit forcing the $[0,0]$ element to 1 in the modulation matrix in (a). Figure 43(b) shows the 17,36 , and 62 variable model fit intensity modulation terms without any constraints modulation matrix normalization.

\subsection{D.4 Polarizer Mueller Matrices and Normalization}

$$
\begin{aligned}
& \mathbf{M}_{i j}=\frac{1}{2}\left(\begin{array}{cccc}
T_{x}+T_{y} & T_{x}-T_{y} & 0 & 0 \\
T_{x}-T_{y} & T_{x}+T_{y} & 0 & 0 \\
0 & 0 & 2 \sqrt{T_{x} T_{y}} & 0 \\
0 & 0 & 0 & 2 \sqrt{T_{x} T_{y}}
\end{array}\right) \text {, } \\
& \mathbf{M}_{i j}=\frac{T_{x}}{2}\left(\begin{array}{cccc}
1+\frac{T_{y}}{T_{x}} & 1-\frac{T_{y}}{T_{x}} & 0 & 0 \\
1-\frac{T_{y}}{T_{x}} & 1+\frac{T_{y}}{T_{x}} & 0 & 0 \\
0 & 0 & 2 \sqrt{\frac{T_{y}}{T_{x}}} & 0 \\
0 & 0 & 0 & 2 \sqrt{\frac{T_{y}}{T_{x}}}
\end{array}\right), \\
& \mathbf{M}_{i j}=\frac{t_{\mathrm{pol}}}{2}\left(\begin{array}{cccc}
1+p_{y}^{2} & 1-p_{y}^{2} & 0 & 0 \\
1-p_{y}^{2} & 1+p_{y}^{2} & 0 & 0 \\
0 & 0 & 2 p_{y} & 0 \\
0 & 0 & 0 & 2 p_{y}
\end{array}\right) \\
& \mathbf{M}_{i j}=\frac{t_{\mathrm{pol}}\left(1+p_{y}^{2}\right)}{2}\left(\begin{array}{cccc}
1 & \frac{1-p_{y}^{2}}{1+p_{y}^{2}} & 0 & 0 \\
\frac{1-p_{y}^{2}}{1+p_{y}^{2}} & 1 & 0 & 0 \\
0 & 0 & \frac{2 p_{y}}{1+p_{y}^{2}} & 0 \\
0 & 0 & 0 & \frac{2 p_{y}}{1+p_{y}^{2}}
\end{array}\right) .
\end{aligned}
$$

We show the Mueller matrix form used for a partial polarizer from Chipman in Eq. (17). The variables $T_{x}$ and $T_{y}$ correspond to the intensity transmittances along the $X$ and $Y$ axes, respectively, (horizontal and vertical). We divide out the transmission of the $X$ polarization state to get the form in Eq. (18).

A common substitution in solar telescope calibration is to describe the transmission of the more transmissive polarization state as the transmission of the polarizer $\left(t_{\mathrm{pol}}\right)$. There is another substitution for the ratio of horizontal and vertical polarization state transmission as $p_{y}^{2}$. We show the Mueller matrix form used for the CalPol in Eq. (19). We note that this matrix form needs to be further normalized for the $[0,0]$ element to be equal to 1 . If the input Stokes vector is purely unpolarized as $[1,0,0,0]^{\mathrm{T}}$, then we recover an output Stokes vector with a transmission $t_{\mathrm{pol}} / 2$ and a vector $\left[1+p_{y}^{2}, 1-p_{y}^{2}, 0,0\right]^{\mathrm{T}}$. We also note that for a pure $Q$ input polarization of $[1,1,0,0]^{\mathrm{T}}$, we recover a transmission of $t_{\mathrm{pol}}$ with the same pure $Q$ output Stokes vector of $[1,1,0,0]^{\mathrm{T}}$.

The normalized form for the polarizer Mueller matrix is in Eq. (20) with the additional $\left(1+p_{y}^{2}\right)$ term included with the transmission function. We note that around $630 \mathrm{~nm}$ wavelength for the DKIST nominal values, we use $p_{y}^{2}$ of roughly $5 \times 10^{-5}$ and a parallel polarization state transmission of $t_{\mathrm{pol}} \sim 95 \%$ for the calibration optic. Under these circumstances, the normalization puts the fit transmission in error at $0.005 \%$ magnitudes: 


$$
\mathbf{M}_{i j}=t_{\mathrm{pol}}^{2}\left(\begin{array}{cccc}
p_{y}^{2} & 0 & 0 & 0 \\
0 & p_{y}^{2} & 0 & 0 \\
0 & 0 & p_{y}^{2} & 0 \\
0 & 0 & 0 & p_{y}^{2}
\end{array}\right)
$$

Extinction ratio (or contrast ratio) is often defined as the ratio of transmitted intensity through parallel polarizers to the transmitted intensity through crossed polarizers for an unpolarized input beam. We use the normalized form for the polarizer Mueller matrix from Eq. (20) and multiply by the same matrix form for a crossed polarizer with reversed signs in the $[0,1]$ and $[1,0]$ elements. We get the Mueller matrix for crossed polarizers per Eq. (21) as a scaled identity matrix:

$$
\mathbf{M}_{i j}=\frac{t_{\mathrm{pol}}^{2}\left(1+p_{y}^{2}\right)^{2}}{4}\left(\begin{array}{cccc}
1+\left(\frac{1-p_{y}^{2}}{1+p_{y}^{2}}\right)^{2} & 2\left(\frac{1-p_{y}^{2}}{1+p_{y}^{2}}\right) & 0 & 0 \\
2\left(\frac{1-p_{y}^{2}}{1+p_{y}^{2}}\right) & 1+\left(\frac{1-p_{y}^{2}}{1+p_{y}^{2}}\right)^{2} & 0 & 0 \\
0 & 0 & \frac{4 p_{y}^{2}}{\left(1+p_{y}^{2}\right)^{2}} & 0 \\
0 & 0 & 0 & \frac{4 p_{y}^{2}}{\left.\left(1+p_{y}^{2}\right)\right)^{2}}
\end{array}\right)
$$

Under the assumption that only the sign of the $I Q$ and $Q I$ elements is reversed, the matrix takes a diagonal form. The $+Q$ created in one orientation is extinguished in the crossed orientation. The $I I$ term becomes one minus the initial polarizer $I Q$ element squared. This simplifies to the standard form of an identity matrix, but with transmission as $\mathrm{t}_{\mathrm{pol}}^{2} p_{y}^{2}$. We also can compute the Mueller matrix for two parallel polarizers as the square of Eq. (20) to get Eq. (22). Note that the $I Q$ and $Q I$ terms are now close to 1 for small values of $p_{y}^{2}$. The $U U$ and $V V$ terms are also small and they can be approximated as $4 p_{y}^{2}$.

\subsection{D.5 Polarizer Contrast Look-up Table Values}

$$
\begin{gathered}
E=\frac{2 p_{y}^{2}}{1+p_{y}^{4}}, \\
C=\frac{1+p_{y}^{4}}{2 p_{y}^{2}} \sim \frac{1}{2 p_{y}^{2}} .
\end{gathered}
$$

Correcting a telescope system model including transmission losses and imperfect contrast is straightforward. By taking the ratio of the parallel and crossed polarizer positions for metrology on the DKIST CalPols, we can derive the extinction ratio and the contrast. The $I I$ elements of Eq. (21) for crossed and Eq. (22) for parallel are easily expanded and simplified. Equation (23) shows the extinction ratio as $E$. Equation (24) shows the contrast ratio as $C$. As an example, for $p_{y}$ of 0.1 , we compute a contrast ratio of 5000 to 1 and a transmission ratio of $0.02 \%$. In Fig. 44, we show the look-up table for $p_{y}$ on converted to contrast in (a) and the transmission function modification term $\left(1+p_{y}^{2}\right)^{2}$ in (b). For now, we populate the DKIST tables with values derived using our lab metrology tools. More complex techniques to separate the polarization influence of the lab analyzer from the DKIST CalPol are possible with future assessments of the DKIST polarizers to be published in the near future.

\section{Appendix E. GOS Calibration Lamp: Stability \& Intensity}

The DKIST ALS is able to provide $0.2 \%$ to $4 \%$ of the solar disk center brightness to coudé focal planes. We were able to derive a spectral ratio of the GOS calibration lamp flux to the DKIST solar beam delivered to the coudé laboratory using NCSP. We recorded counts for the lamp and the Sun in the same optical configuration with the same integration time and the same filter color 
balancing within an hour duration test in August 2020. A common background frame was subtracted from both VIS and NIR spectrographs. Figure 45 shows the detected count ratio of lamp flux divided by solar flux after dark subtraction. We note that the detected count levels were at least thousands for the lamp and tens to 160 thousand for the Sun on the VIS channel, well within the linearity range of the sensors. We also confirmed linear response of these USB spectrographs to better than $1 \%$ over the read noise limited count range in the laboratory. The lamp achieves a brightness of higher than $0.3 \%$ of the solar beam for wavelengths longer than $500 \mathrm{~nm}$. The lamp is brighter than $1 \%$ of the Sun for wavelengths longer than $650 \mathrm{~nm}$. The smooth curve underlying spectral features represents the expected black body ratio of a 5500-K Sun to a 3500-K lamp bulb. Obvious solar spectral features are seen at low levels. Atmospheric absorption bands are present and expected from the $\sim 60-\mathrm{m}$ optical path from the GOS lamp to NCSP compared to the 10,000-ft summit atmosphere.

\section{Acknowledgments}

This work was supported by the National Science Foundation's (NSF's) Daniel K. Inouye Solar Telescope (DKIST) project. DKIST is operated by NSF's National Solar Observatory under a cooperative agreement with the Association of Universities for Research in Astronomy, Inc. (AURA). DKIST is located on land of spiritual and cultural significance to Native Hawaiian people. The use of this important site to further scientific knowledge is done so with appreciation and respect. We are honored to have the opportunity to conduct astronomical research on Haleakala on the Island of Maui in Hawaií. We recognize and acknowledge the very significant cultural role and reverence that this site has to the Native Hawaiian Community. The Polarimetry Analysis and Calibration (PA\&C) team worked diligently to design, install, test, and assist with the various metrology tools available to the community. This includes scientific input from David Elmore and Christian Beck as well as the PA\&C team: Andy Ferayorni, Scott Gregory, Austin Kootz, Chris Runyan, Wes Cole, and many others. This project would not have been possible without the massive effort of the DKIST construction team. In particular, the summit team for their dedication and for dealing with all the challenges from building such a complex custom facility. Amanda White acknowledges funding from the George Ellery Hale Graduate Fellowship at the University of Colorado Boulder. The authors would like to thank Lucas Tarr for discussing many variable fitting techniques and for having a very large white board full of equations. We thank Moxtek and their staff Eric Gardner and Alex Gao for engagement with us on upgrade projects and many technical conversations about polarizer performance; we also thank Meadowlark Optics staff, the metrology and analysis from Michael Kraemer, assistance from Larry Opila on metrology equipment, spectroscopy, data analysis; and also for numerous lengthy physics discussions with the one and only Tom Baur. This research made use of Astropy, a community-developed core Python package for Astronomy. ${ }^{107,108}$

\section{References}

1. T. R. Rimmele et al., "The Daniel K. Inouye Solar Telescope-observatory overview," Solar Phys. 295, 172 (2020).

2. J. P. McMullin et al., "Construction status of the Daniel K. Inouye Solar Telescope," Proc. SPIE 9145, 914525 (2014).

3. S. L. Keil et al., "ATST: the largest polarimeter," in Solar Polarization 6. Proc. Conf., Maui, Vol. 437, p. 319 (2011).

4. T. R. Rimmele et al., "Instrumentation for the advanced technology solar telescope," Proc. SPIE 5492, 944 (2004).

5. J. Marino, E. Carlisle, and D. Schmidt, "Simulation of DKIST solar adaptive optics system," Proc. SPIE 9909, 99097C (2016).

6. J. P. McMullin et al., "Construction status of the Daniel K. Inouye Solar Telescope," Proc. SPIE 9906, 99061B (2016).

7. L. C. Johnson et al., "Status of the DKIST system for solar adaptive optics," Proc. SPIE 9909, 99090Y (2016). 
8. D. F. Elmore, S. R. Sueoka, and R. Casini, "Performance of polarization modulation and calibration optics for the Daniel K. Inouye Solar Telescope," Proc. SPIE 9147, 91470F (2014).

9. D. F. Elmore et al., "The Daniel K. Inouye Solar Telescope first light instruments and critical science plan," Proc. SPIE 9147, 914707 (2014).

10. P. Sekulic et al., "Daniel K. Inouye Solar Telescope optical alignment plan," Proc. SPIE 9906, 990653 (2016).

11. R. Hubbard, S. Craig, and R. Kneale, "Daniel K. Inouye Solar Telescope systems engineering update," Proc. SPIE 9911, 99112F (2016).

12. S. R. Sueoka, R. A. Chipman, and D. F. Elmore, "Characterization of DKIST retarder components with polarization ray tracing," Proc. SPIE 9293, 929308 (2014).

13. D. F. Elmore et al., "Utilization of redundant polarized solar spectra to infer the polarization properties of the new generation of large aperture solar telescopes," Proc. SPIE 7735, 77354E (2010).

14. D. Elmore, "Telescope calibration using polarization calibration data," Astron. Nachr. 331(6), 655-657 (2010).

15. S. Sueoka, "Polarization optical components of the Daniel K. Inouye Solar Telescope," $\mathrm{PhD}$ thesis, University of Arizona (2016).

16. J. C. Guzman and J. Ibsen, Eds., Motor Control for 0.1-Meter Diameter Crystal Retarders on the Daniel K. Inouye Solar Telescope, SPIE Press (2018).

17. A. Ferayorni et al., "DKIST controls model for synchronization of instrument cameras, polarization modulators, and mechanisms," Proc. SPIE 9152, 91520Z (2014).

18. W. Schmidt et al., "End-to-end simulations of the visible tunable filter for the Daniel K. Inouye Solar Telescope," Proc. SPIE 9908, 99084N (2016).

19. W. Schmidt et al., "A two-dimensional spectropolarimeter as a first-light instrument for the Daniel K. Inouye Solar Telescope," Proc. SPIE 9147, 91470E (2014).

20. F. Wöger, "DKIST visible broadband imager interference filters," Proc. SPIE 9147, 91479I (2014).

21. A. Ferayorni et al., "Bottom-up laboratory testing of the DKIST visible broadband imager (VBI)," Proc. SPIE 9911, 991106 (2016).

22. A. Beard, B. Cowan, and A. Ferayorni, "DKIST visible broadband imager data processing pipeline," Proc. SPIE 9152, 91521J (2014).

23. P. Sekulic et al., "DKIST visible broadband imager alignment in laboratory: first results," Proc. SPIE 9908, 99085A (2016).

24. A. Ferayorni, "Instrument control software for the visible broadband imager using ATST common services framework and base," Proc. SPIE 8451, 845113 (2012).

25. F. Wöger and A. Ferayorni, "Accelerated speckle imaging with the ATST visible broadband imager," Proc. SPIE 8451, 84511C (2012).

26. W. R. McBride et al., "ATST visible broadband imager," Proc. SPIE 8446, 84461B (2012).

27. H. Socas-Navarro et al., "High precision polarimetry with the Advanced Technology Solar Telescope," Proc. SPIE 5901, 590105 (2005).

28. K. Richards et al., "The adaptive optics and wavefront correction systems for the Advanced Technology Solar Telescope," Proc. SPIE 7736, 773608 (2010).

29. S. Berukoff et al., "Petascale cyberinfrastructure for ground-based solar physics: approach of the DKIST data center," Proc. SPIE 9913, 99131F (2016).

30. F. T. Watson et al., "Calibration development strategies for the Daniel K. Inouye Solar Telescope (DKIST) data center," Proc. SPIE 9910, 99101G (2016).

31. C. Mayer, S. Wampler, and B. Goodrich, "World coordinate information for the Daniel K. Inouye Solar Telescope," Proc. SPIE 9913, 99130S (2016).

32. W. H. Schubert, E. Petrak, and T. G. Baur, "Measurement of polarization assemblies for the Daniel K. Inouye Solar Telescope," Proc. SPIE 9369, 93690N (2015).

33. A. G. de Wijn et al., "Preliminary design of the visible spectro-polarimeter for the Advanced Technology Solar Telescope," Proc. SPIE 8446, 84466X (2012).

34. J. Sánchez-Capuchino et al., "Current concept for the 4m European Solar Telescope (EST) optical design," Proc. SPIE 7733, 773336 (2010). 
35. F. C. M. Bettonvil et al., "The polarization optics for the European Solar Telescope (EST)," Proc. SPIE 7735, 77356I (2010).

36. M. Collados et al., "European Solar Telescope: project status," Proc. SPIE 7733, 77330H (2010).

37. M. De Juan Ovelar et al., "Instrumental polarisation at the Nasmyth focus of the E-ELT," Astron. Astrophys. 562, A8 (2014).

38. F. Joos et al., "Reduction of polarimetric data using Mueller calculus applied to Nasmyth instruments," Proc. SPIE 7016, 70161I (2008).

39. C. U. Keller and F. Snik, "Polarimetry from the Ground Up," in Solar Polarization 5: In Honor of Jan Stenflo ASP Conf. Ser., Vol. 405, p. 371 (2009).

40. C. U. Keller et al., "EPOL: the exoplanet polarimeter for EPICS at the E-ELT," Proc. SPIE 7735, 77356G (2010).

41. C. U. Keller, "Solar polarimetry close to the diffraction limit," Proc. SPIE 4843, 100 (2003).

42. M. Rodenhuis et al., "The extreme polarimeter: design, performance, first results and upgrades," Proc. SPIE 8446, 84469I (2012).

43. R. Roelfsema et al., "The ZIMPOL high-contrast imaging polarimeter for SPHERE: design, manufacturing, and testing," Proc. SPIE 7735, 77354B (2010).

44. J. Sánchez Almeida, "Instrumental polarization in the focal plane of telescopes. 2: effects induced by seeing," Astron. Astrophys. 292, 713-721 (1994).

45. J. Sánchez Almeida and V. Martinez Pillet, "Instrumental polarization in the focal plane of telescopes," Astron. Astrophys. 260, 543-555 (1992).

46. J. Sánchez Almeida, V. Martinez Pillet, and A. D. Wittmann, "The instrumental polarization of a Gregory-Coude Telescope," Solar Phys. 134, 1-13 (1991).

47. W. Schmidt et al., "POLIS: a spectropolarimeter for the VTT and for GREGOR," Astron. Nachr. 324, 300 (2003).

48. F. Snik et al., "Design of a full-Stokes polarimeter for VLT/X-shooter," Proc. SPIE 8446, 844625 (2012).

49. F. Snik et al., "The upgrade of HARPS to a full-Stokes high-resolution spectropolarimeter," Proc. SPIE 7014, 701400 (2008).

50. F. Snik, "Calibration strategies for instrumental polarization at the 10-5 level," Proc. SPIE 6269, 62695P (2006).

51. H. Socas-Navarro et al., "Characterization of telescope polarization properties across the visible and near-infrared spectrum. Case study: the Dunn Solar Telescope," Astron. Astrophys. 531, A2 (2011).

52. H. Socas-Navarro, "Polarimetric calibration of large-aperture telescopes. II. Subaperture method," J. Opt. Soc. Am. A 22, 907 (2005).

53. H. Socas-Navarro, "Polarimetric calibration of large-aperture telescopes. I. Beamexpansion method," J. Opt. Soc. Am. A 22, 539 (2005).

54. P. Spano et al., "Optical design of CAOS: a high-resolution spectropolarimeter for the Catania Astrophysical Observatory 0.91-m telescope," Proc. SPIE 5492, 373-380 (2004).

55. K. G. Strassmeier et al., "PEPSI: the Potsdam Echelle Polarimetric and Spectroscopic Instrument for the LBT," Proc. SPIE 7014, 70140N (2008).

56. K. G. Strassmeier et al., "PEPSI spectro-polarimeter for the LBT," Proc. SPIE 4843, 180-189 (2003).

57. J. Tinbergen, "Accurate optical polarimetry on the Nasmyth platform," Publ. Astron. Soc. Pac. 119, 1371-1384 (2007).

58. C. Beck et al., "A polarization model for the German Vacuum Tower Telescope from in situ and laboratory measurements," Astron. Astrophys. 443, 1047-1053 (2005).

59. C. Beck et al., "Polarimetric Littrow Spectrograph-instrument calibration and first measurements," Astron. Astrophys. 437, 1159-1167 (2005).

60. L. Kleint et al., "GREGOR: optics redesign and updates from 2018-2020," Astron. Astrophys. 641, A27-10 (2020).

61. D. M. Harrington and S. R. Sueoka, "Polarization modeling and predictions for DKIST, part 1: telescope and example instrument configurations," J. Astron. Telesc. Instrum. Syst. 3, 018002 (2017). 
62. D. M. Harrington, J. R. Kuhn, and A. L. Ariste, "Daytime sky polarization calibration limitations," J. Astron. Telesc. Instrum. Syst. 3, 018001 (2017).

63. D. M. Harrington and S. R. Sueoka, "Polarization modeling and predictions for DKIST part 3: focal ratio and thermal dependencies of spectral polarization fringes and optic retardance," J. Astron. Telesc. Instrum. Syst. 4(1), 018006 (2018).

64. D. W. Berreman, "Optics in stratified and anisotropic media: $4 \times 4$-matrix formulation," J. Opt. Soc. Am. 62, 502 (1972).

65. M. W. McCall, I. J. Hodgkinson, and Q. Wu, Birefringent Thin Films and Polarizing Elements, Vol. 1, 2nd ed., Imperial College Press, London (2014).

66. D. M. Harrington and S. R. Sueoka, "Polarization modeling and predictions for DKIST part 4: calibration accuracy over field of view, retardance spatial uniformity, and achromat design sensitivity," J. Astron. Telesc. Instrum. Syst. 4, 044006 (2018).

67. D. M. Harrington, S. R. Sueoka, and A. J. White, "Polarization modeling and predictions for DKIST part 5: impacts of enhanced mirror and dichroic coatings on system polarization calibration," J. Astron. Telesc. Instrum. Syst. 5, 038001 (2019).

68. M. Semel, "Spectropolarimetry and polarization-dependent fringes," Astron. Astrophys. 401, 1-14 (2003).

69. D. Clarke, "Interference effects in compound and achromatic wave plates," J. Opt. A: Pure Appl. Opt. 6, 1041-1046 (2004).

70. D. Clarke, "Effects in polarimetry of interference within wave plates," Astron. Astrophys. 434, 377-384 (2005).

71. D. Clarke, "Interference effects in single wave plates," J. Opt. A: Pure Appl. Opt. 6, 1036-1040 (2004).

72. D. Clarke, "Interference effects in Pancharatnam wave plates," J. Opt. A: Pure Appl. Opt. 6, 1047-1051 (2004).

73. D. Clarke, Stellar Polarimetry, John Wiley \& Sons, Weinheim (2009).

74. O. S. Heavens, Optical Properties of Thin Solid Films (Dover Books on Physics), Dover, New York (1965).

75. D. K. Aitken and J. H. Hough, "Spectral modulation, or ripple, in retardation plates for linear and circular polarization," Publ. Astron. Soc. Pac. 113, 1300 (2001).

76. T. J. Harries and I. D. Howarth, "Linear spectropolarimetry of the H $\alpha$ emission line of $\zeta$ Puppis," Astron. Astrophys. 310, 533 (1996).

77. D. M. Harrington et al, "Correcting systematic polarization effects in Keck LRISp spectropolarimetry to 0.05 percent," Publ. Astron. Soc. Pac. 127, 757-775 (2015).

78. F. Snik et al., "A multi-domain full-Stokes polarization modulator that is efficient for 300-2500 nm spectropolarimetry," Proc. SPIE 9613, 96130G (2015).

79. A. Derks, C. Beck, and V. Pillet, "Inferring telescope polarization properties through spectral lines without linear polarization," Astron. Astrophys. 615, A22 (2018).

80. D. M. Harrington et al., "Polarization modeling and predictions for DKIST, part 6: fringe mitigation with polycarbonate modulators and optical contact calibration retarders," J. Astron. Telesc. Instrum. Syst. 6, 038001 (2020).

81. E. Collett, Polarized Light. Fundamentals and Applications, Vol. 1, 1st ed., CRC Press (1992).

82. R. A. Chipman, "Chapter 15: Polarimetry," in Handbook of Optics Volume, M. Bass, Ed., McGraw Hill, New York (2014).

83. R. A. Chipman, Handbook of Optics: Chapter 14, Mueller Matrices, Vol. 1, 3rd ed., McGraw Hill, New York (2010).

84. F. Snik and C. U. Keller, Astronomical Polarimetry: Polarized Views of Stars and Planets, Vol. 1, 2nd ed., Springer, Dordrecht (2013).

85. J. C. del Toro Iniesta, Introduction to Spectropolarimetry, Cambridge University Press, Cambridge (2003).

86. J. C. del Toro Iniesta and M. Collados, "Optimum modulation and demodulation matrices for solar polarimetry," Appl. Opt. 39, 1637 (2000).

87. J. C. del Toro Iniesta and V. Martinez Pillet, "Assessing the behavior of modern solar magnetographs and spectropolarimeters," Astrophys. J. Suppl. Ser. 201, 22 (2012).

88. R. A. Chipman, W. S. T. Lam, and G. Young, Polarized Light and Optical Systems, CRC Press, Boca Raton, Florida (2018). 
89. S. Tomczyk et al., "Wavelength-diverse polarization modulators for Stokes polarimetry," Appl. Opt. 49, 3580-3586 (2010).

90. A. G. D. Wijn et al., "Wavelength-diverse polarization modulators for stokes polarimetry," in Solar Polarization 6. Proc. Conf., Maui, Vol. 437, p. 413 (2011).

91. A. Skumanich et al., "The calibration of the advanced Stokes polarimeter," Astrophys. J. Suppl. Ser. 110, 357-380 (1997).

92. F. Snik, T. Karalidi, and C. Keller, "Spectral modulation for full linear polarimetry," Appl. Opt. 48(7), 1337-1346 (2009).

93. R. Chipman, "Classification of depolarizing Mueller Matrices," in Front. Opt., Washington, D.C. (2006).

94. R. A. Chipman, "Depolarization," Proc. SPIE 3754, 14-20 (1999).

95. R. A. Chipman, "Degrees of freedom in depolarizing Mueller matrices," Proc. SPIE 6682, 66820I (2007).

96. R. A. Chipman, "Metrics for depolarization," Proc. SPIE 5888, 58880L (2005).

97. R. A. Chipman, "Depolarization index and the average degree of polarization," Appl. Opt. 44, 2490-2495 (2005).

98. R. A. Chipman, "Depolarization in the Mueller calculus," Proc. SPIE 5158, 184-192 (2003).

99. R. A. Chipman and S.-Y. Lu, "Decomposition of Mueller matrices," in Opt. Sci. Eng. Instrum. '97, Vol. 3120, pp. 385-396 (1997).

100. B. Deboo, J. Sasian, and R. Chipman, "Degree of polarization surfaces and maps for analysis of depolarization," Opt. Express 12, 4941 (2004).

101. H. D. Noble, S. C. McClain, and R. A. Chipman, "Mueller matrix roots depolarization parameters," Appl. Opt. 51, 735 (2012).

102. H. D. Noble, S. McClain, and R. A. Chipman, "Mueller matrix roots," PhD thesis, The University of Arizona (2011).

103. M. Makita, Y. Funakoshi, and Y. H. S. Polarimetry, "Polarization of the domeless solar telescope of the Hida observatory (preliminary report)," in Solar Polarimetry NSO/SP Summer Workshop 11, pp. 198-201 (1991).

104. J. Kiyohara et al., "Calibration of the instrumental polarization of the Domeless Solar Telescope at the Hida Observatory," Proc. SPIE 5492, 1778-1785 (2004).

105. Y. Hanaoka, "Spectropolarimetry with the Hida Domeless Solar Telescope," Publ. Astron. Soc. Jpn. 61(2), 357-365 (2009).

106. C. Capitani et al., "Polarization properties of a Zeiss-type coelostat: the case of the solar tower in Arcetri," Solar Phys. 120(1), 173-191 (1989).

107. Astrophy Collaborationet al., "The Astropy Project: building an inclusive, open-science project and status of the v2.0 core package," Astron J. 156, 123 (2018).

108. Astrophy Collaborationet al., "Astropy: a community Python package for astronomy," Astron. Astrophys. 558, A33 (2013).

Biographies of the authors are not available. 